$2012-12-20$

\title{
Local signal regularity and smoothness as a means for seismic $Q$ estimation
}

\author{
Izadi, Hormoz
}

Izadi, H. (2012). Local signal regularity and smoothness as a means for seismic Q estimation (Unpublished master's thesis). University of Calgary, Calgary, AB. doi:10.11575/PRISM/26779 http://hdl.handle.net/11023/378

Downloaded from PRISM Repository, University of Calgary 


\section{UNIVERSITY OF CALGARY}

Local signal regularity and smoothness as a means

for seismic $Q$ estimation

by

Hormoz Izadi

\section{A DISSERTATION}

SUBMITTED TO THE FACULTY OF GRADUATE STUDIES

IN PARTIAL FULFILLMENT OF THE REQUIREMENTS FOR THE

DEGREE OF MASTER OF SCIENCE

DEPARTMENT OF GEOSCIENCE

CALGARY, ALBERTA

December, 2012

(C) Hormoz Izadi 2012 


\section{UNIVERSITY OF CALGARY FACULTY OF GRADUATE STUDIES}

The undersigned certify that they have read, and recommend to the Faculty of Graduate Studies for acceptance, a dissertation entitled "Local signal regularity and smoothness as a means for seismic $Q$ estimation" submitted by Hormoz Izadi in partial fulfillment of the requirements for the degree of MASTER OF SCIENCE. 


\begin{abstract}
In seismic signal analysis, irregular structures and points of sharp variation contain critical information, thus making the study of a signal's local properties an appropriate mechanism for obtaining information from seismic data. The local regularity of a seismic event is determined by the wavelet transform modulus maxima and the associated Lipschitz exponent. As a means of classifying regularities of a signal and estimating the associated Lipschitz exponent, the linear and non-linear Mallat-Hwang-Zhong (MHZ) signal model based on the wavelet theory is reviewed and developed.

For isolated seismic events, resembling a delta function or a Heaviside function, the linear MHZ model is used to estimate the associated Lipschitz exponent and subsequently verify the theoretical properties of the exponent. However for practical settings, in particular, band-limited signal events, the more complex non-linear MHZ signal model must be applied in order to estimate the local regularity and the additional smoothness parameter.

Based on the synthetic vertical seismic profile (VSP) modelling, a relatively complicated mathematical mapping between the Lipschitz exponent and seismic quality factor $Q$ is obtained. However, analysing the smoothness parameter results in an invertible power law relation between the aforementioned parameter and Q. Applying the non-linear MHZ model to the Ross Lake VSP field data captures the general absorption trend estimated by Zhang and Stewart (2006). Furthermore, the power law relation provides geophysically reasonable $Q$ values comparable to the estimated values using traditional methods, such as the steepest descent. However, for a more robust mathematical relation between the Lipschitz exponent, smoothness parameter and seismic quality factor $Q$, additional theoretical and field data analysis is required.
\end{abstract}




\section{Acknowledgements}

First and foremost, I would like to thank my supervisors Dr. Kris Innanen and Dr. Michael Lamoureux for their guidance, support, valuable insight, comments and encouragement throughout my program.

I would also like to express my gratitude to Yousry Elsabrouty for his timely advice and feedback.

Thanks to CREWES Faculty members, staff and students, especially Rolf Maier, Helen Isaac, David Henley, Glen Young, Faranak Mahmoudian, Mahdi Al-Mutlaq, Hassan Khaniani, Peng Cheng, David Cho and Ben Wards for their help and advice.

Last but not least, I would like to thank my parents for their continued support and words of encouragement. Thank you! 


\section{Table of Contents}

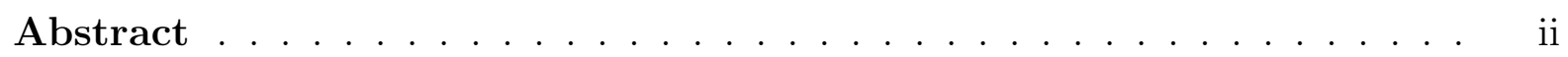

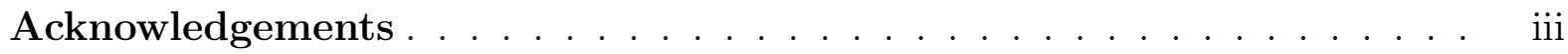

Table of Contents . . . . . . . . . . . . . . . . . . . iv

List of Tables . . . . . . . . . . . . . . . . . . . . . .

List of Figures . . . . . . . . . . . . . . . . . . . . . . . . . . . . . . . . . . . . . . .

List of Symbols . . . . . . . . . . . . . . . . . . . . . . . viii

1 Introduction . . . . . . . . . . . . . . . . . . . 1

1.1 Statement of the Problem . . . . . . . . . . . . . . . . . 1

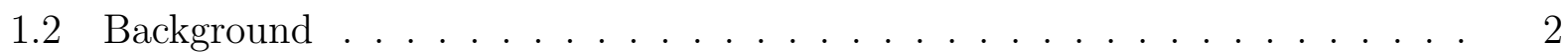

1.2.1 Time-Frequency Analysis . . . . . . . . . . . . . . . . . . 2

1.2.2 Seismic Attenuation and $Q \ldots \ldots \ldots \ldots$

$1.3 Q$ estimation methods . . . . . . . . . . . . . . . . . 6

1.3.1 Amplitude decay method . . . . . . . . . . . . . . . 6

1.3.2 Wavelet modelling method . . . . . . . . . . . . . . 6

1.3.3 Phase modelling method . . . . . . . . . . . . . . 6

1.3.4 Spectral ratio method . . . . . . . . . . . . . . . . 7

1.4 Seismic signal smoothness \& Lipschitz regularity . . . . . . . . . . . . . . . 7

1.5 Vertical seismic profile (VSP) data . . . . . . . . . . . . . . . 8

1.6 Thesis objective . . . . . . . . . . . . . . . . . . . . . . . . . . . 10

1.7 Thesis outline . . . . . . . . . . . . . . . . . . . . . 10

2 Continuous wavelet transforms and Lipschitz regularity . . . . . . . . . . . . 12

2.1 Time-frequency methods . . . . . . . . . . . . . . . . . . . 12

2.1.1 Wavelet tranforms . . . . . . . . . . . . . . 16

2.2 Properties of the continuous wavelet transform (CWT) . . . . . . . . . . 20

2.2.1 Superposition . . . . . . . . . . . . . . . 20

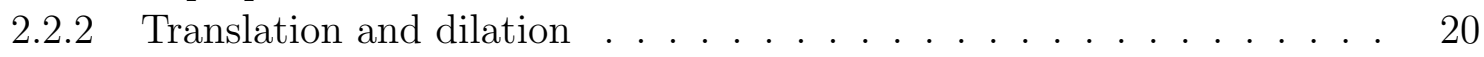

2.2 .3 Local regularity . . . . . . . . . . . . . . . . . . 20

2.3 The Lipschitz exponent . . . . . . . . . . . . . . . . . . . . . . . . . . . . 21

2.4 Estimating Lipschitz regularity . . . . . . . . . . . . . . . . . . . . . . . . . . . . . . . . . . . . . .

2.4.1 The Mallat-Hwang-Zhong signal model . . . . . . . . . . . . 23

2.4.2 Implementation of the MHZ model: steepest descent . . . . . . . . . 25

2.4.3 Implementation of the MHZ model: least-squares . . . . . . . . . . . 27

3 Algorithms for estimating regularity and smoothness . . . . . . . . . . . . 29

3.1 The Lipschitz regularity of a spike . . . . . . . . . . . . . . . . . . . . . . . . . . . . . . . . 29

3.2 The Lipschitz regularity of a step and ramp function . . . . . . . . . . . . . . . . . . . . . . . . . 33

3.3 The effect of closely-spaced events . . . . . . . . . . . . . . . . . . . . . . . . . . .

4 Determining Q from the regularity of VSP data: synthetic study . . . . . . . 40

4.1 Vertical seismic profile (VSP) data . . . . . . . . . . . . . . . 40

4.2 The regularity and smoothness of VSP events . . . . . . . . . . . . . 41

4.2 .1 Steepest descent estimation . . . . . . . . . . . . . . . . . . . . . . . 42

4.2 .2 Scales to be used and avoided . . . . . . . . . . . . . . . . . . 43

4.3 A re-arrangement of the model . . . . . . . . . . . . . . . . . 46 
4.4 The relationship between MHZ model parameters and $Q \ldots \ldots$. . . . . 48

4.4.1 The relationship between the Lipschitz regularity $\alpha$ and $Q \ldots$. . . 48

4.4.2 The relationship between smoothness, $\sigma$, and $Q \ldots \ldots . \ldots 54$

4.4.3 $Q$ estimation from MHZ model parameters . . . . . . . . . . . . 62

4.5 Towards application to field data . . . . . . . . . . . . . . . . . . . . . . . . . . . . 65

4.5 .1 Noise . . . . . . . . . . . . . . . . . . 65

4.5.2 A Procedure for application to field data . . . . . . . . . . . . 65

5 Application to the Ross Lake VSP field data set . . . . . . . . . . . . . . . 69

5.1 Study area . . . . . . . . . . . . . . . . . . . . 69

5.2 Data set . . . . . . . . . . . . . . . . . . 70

$5.2 .1 Q$ in the Ross Lake VSP data . . . . . . . . . . . . . . 73

5.2.2 Estimating Ross Lake MHZ parameters and $Q \ldots \ldots$. . . . . 76

5.2 .3 Discussion . . . . . . . . . . . . . . . . . . . 80

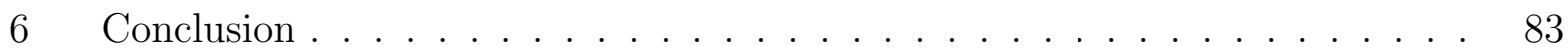




\section{List of Tables}

3.1 Corresponding maxima modulus values at each scale, for the delta function . 33

3.2 Modulus maxima values at each scale, for the Heaviside function . . . . . . . 36

3.3 Modulus maxima values at each scale index, for the Ramp function . . . . . 36

4.1 Estimated regularity and smoothness values using steepest descent . . . . . . 42

4.2 Estimated $\alpha$ and $\sigma$ values using steepest descent for fixed depth and varying $Q$ values .................................. 44

4.3 Estimated $\alpha$ and $\sigma$ using steepest descent for fixed receiver depth and $Q$ value 44

4.4 Estimated $\alpha$ and $\sigma$ values using least squres method corresponding to direct arrival with fixed wave velocity and $Q$ values . . . . . . . . . . . 50

4.5 Corresponding values mapping $\sigma$ to $Q$ using least squares method . . . . . . 60

4.6 Corresponding values mapping $\sigma$ to $Q$ using weighted least squares method . 60

4.7 Corresponding values mapping $\sigma$ to $Q$ using least squares method from loga-

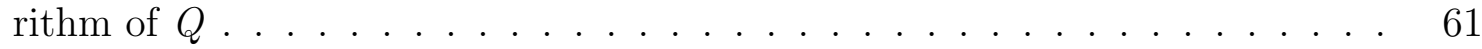

4.8 Estimated $Q$ values . . . . . . . . . . . . . . . . . . 64

5.1 Acquisition parameters for the Ross Lake VSP survey . . . . . . . . . . . . . 73

5.2 Corresponding depth and local $\sigma$ values . . . . . . . . . . . . . . . 77

5.3 Coefficient values obtained from synthetic modelling . . . . . . . . . . 78

5.4 Estimated $Q$ values from from downgoing P-wave using spectral ratio method 80 


\section{List of Figures and Illustrations}

1.1 VSP experiment and the effects of absorption on a seismic pulse . . . . . . 9

2.1 Visual description of the short-time Fourier transforms . . . . . . . . . .

2.2 Visual comaparison between wavelet transform and the short-time Fourier transform . . . . . . . . . . . . . . . . . . 18

2.3 Wavelet scaling functions vs wavlets . . . . . . . . . . . . . . . . . . . 19

2.4 Original signal and corresponding continuous wavelet transform . . . . . . . 22

2.5 Illustration of steepest descent . . . . . . . . . . . . . . . 27

3.1 Original input signal (delta function) . . . . . . . . . . . . . . . . 30

3.2 Continuous wavelet transform of delta function and corresponding modulus maxima values . . . . . . . . . . . . . . . . . 31

3.3 Logarithm of maxima modulus against scale index for delta function . . . . . 32

3.4 Two parameter objective function . . . . . . . . . . . . . . . . . 32

3.5 Wavelet transform and corresponding modulus maxima values at each scale for Heaviside function . . . . . . . . . . . . . . . . . . . . . . . . 34

3.6 Logarithm of modulus maxima values against scale index for Heaviside function 35

3.7 Logarithm of modulus maxima values against scale index for Ramp function 36

3.8 Logarithm of modulus maxima against scale index for pulse with increasing smoothness . . . . . . . . . . . . . . . . 37

3.9 Closely spaced Gaussians representing the input signal . . . . . . . . . . . 38

3.10 Corresponding modulus maxima values for two closely spaced events . . . . . 38

3.11 Logarithm of modulus maxima against scale index corresponding to a pulse with increasing absorption . . . . . . . . . . . . . . . . . . 39

4.1 Zero-offset VSP field layout . . . . . . . . . . . . . . . . . 41

4.2 Direct arrivals corresponding to receiver depth with varying $Q$ values . . . . 43

4.3 Logarithm of modulus maxima values vs scale index $j \ldots \ldots$. . . . . . . . . 45

4.4 Logarithm of modulus maxima values vs scale index $j$, for fixed depth and $Q$ value . . . . . . . . . . . . . . . . . . 46

4.5 Direct arrivals corresponding to varying receiver depth values . . . . . . . . . 48

4.6 Logarithm of modulus maxima values against scale index for varying receiver depth values . . . . . . . . . . . . . . . . . . . . . . . 49

4.7 Corresponding plot of $\alpha$ against $Q$ with estimated fit . . . . . . . . . 52

4.8 Plot of $\alpha$ against $Q$ with estimated fit corresponding to increasing receiver

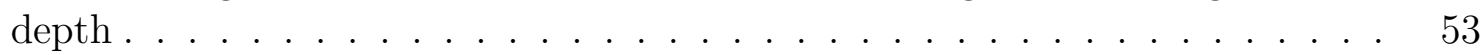

4.9 Corresponding plot of $\alpha$ against $Q$ for fixed $Q$ and varying receiver depth values 54

4.10 Corresponding plot of $\sigma$ against $Q \ldots \ldots \ldots \ldots$

4.11 Plot of $\sigma$ against $Q$ corresponding to fixed receiver depth . . . . . . . . . 58

4.12 Plot of $\sigma$ against $Q$ corresponding to increasing receiver depth value . . . . . 59

4.13 Corresponding plot of $\sigma$ against $Q$ for varying receiver depth values . . . . . 60

4.14 Errors associated with least squares and weighted least squares approximation 61

4.15 Comparison of absolute errors values . . . . . . . . . . . . . . . . 62 
4.16 Comparison of absolute errors associated with least squares and weighted least squares method for varying receiver depth values . . . . . . . . . . . . . 63

4.17 Direct arrivals (containing noise) corresponding to varying receiver depth values 67

4.18 Logarithm of modulus maxima against scale index for data containing noise 68

5.1 Ross Lake heavy oil field . . . . . . . . . . . . . . . . . . . . . . . . . . 69

5.2 Estimated $\mathrm{P}$ and $\mathrm{S}$ wave velocities from zero-offset VSP . . . . . . . . . . 71

5.3 Regional stratigraphic chart in southwest Saskatchewan . . . . . . . . . . . . 72

5.4 Downgoing P-wave corresponding to the far-offset VSP survey . . . . . . . . 74

5.5 Trace of direct $\mathrm{P}$-wave arrivals . . . . . . . . . . . . . . . . . . 74

5.6 Logarithm of modulus maxima against scale index corresponding to Ross Lake VSP data . . . . . . . . . . . . . . . . . . . . . . 75

5.7 Estimated $\sigma$ values against receiver number f . . . . . . . . . . . 78

5.8 Estimated local $\sigma$ values against receiver number . . . . . . . . . . . . . . 79

5.9 Estimated $Q$ values corresponding to Ross Lake VSP data . . . . . . . . . . 81

5.10 Spectral ratio plot of downgoing P-wave . . . . . . . . . . . . . . 82 


\section{List of Symbols, Abbreviations and Nomenclature}

Symbol
$\alpha$
$\sigma$
$Q$
$\psi$
$\mathbf{W} f(.$.
$c$
$\omega$
$s$
$j$

Symbol
Definition

Local signal regularity - Lipschitz exponent

Local signal smoothness

Seismic quality factor

Mother wavelet

Wavelet transform of function $f$

Wave velocity

Temporal frequency

Wavelet scale

Wavelet scale index 


\section{Chapter 1}

\section{Introduction}

\subsection{Statement of the Problem}

Singularities and points of sharp variation (within signals) carry critical information that are typically amongst the most important features for analysing properties of transient signals or images (Mallat and Zhong, 1992). Points of sharp variation created by shadows, occlusions, highlights are typically located at boundaries of image structures and contain different intensity profiles (Mallat and Zhong, 1992). In seismic signal analysis, regions of abrupt change classifiable as "edges" contain a considerable amount of a signal's information, thus making edge detection a potentially appropriate and efficient tool for obtaining information from seismic data (Innanen, 2003). Edge detection requires analysis of local properties of the corresponding edges.

Traditionally, the Fourier transform has been the main mathematical tool and technique for analysing singularities and irregular structures. However, a major drawback lies in the fact that the Fourier transform generally provides a description of a signal's overall singularity, thus it is not well suited for finding spatial distributions and locations of singularities (Mallat and Zhong, 1992; Mallat and Hwang, 1992).

Applying advanced mathematical techniques, namely continuous wavelet transform, enables us to obtain the modulus maxima from seismic data and estimate the Lipschitz exponents which in turn allows us to measure the local regularity of functions and differentiate the intensity profile of different edges (Mallat and Zhong, 1992; Mallat and Hwang, 1992).

Several important physical processes can in principle affect the local regularity of a reflected event in a seismic trace: processes of absorption/wave attenuation, and reflections from targets composed of thin (sub-wavelength) layers. It is generally understood that due 
to absorption, the energy of seismic waves propagating through an anelastic medium would dissipate over a given distance. As a result, transient waveforms are distorted as they propagate through such media; progressive loss of amplitudes and changes of phase are typically encountered (Kjartansson, 1979; Zhang, 2008). The overall effect of seismic attenuation is described by the dimensionless quality factor $Q$, with studies in seismic data processing concentrating on modelling, estimation or compensation (Innanen, 2003). In practical terms, estimation and compensation can potentially enhance the resolving power of the seismic data. A robust estimation of the Lipschitz exponents from seismic data, alongside with prior geological information, could potentially lead to processing and inversion algorithms able to discern and characterise such targets. Algorithms of this kind would be of significant scientific and economic value.

\subsection{Background}

To investigate the relationship between attenuation and the corresponding local regularity and smoothness of a seismic signal, we implement and adapt a signal model based on the continuous wavelet transform, and apply it to the synthetic and field vertical seismic profile. Such a procedure requires a comprehensive understanding of time-frequency analysis, seismic absorption and $Q$ estimation methods, local signal properties and the vertical seismic profiles.

\subsubsection{Time-Frequency Analysis}

In the field of signal analysis, which involves the study and characterisation of basic signal properties, it is often critical and beneficial to mathematically expand and study a signal in a set of differing representations characterised by the physical quantity vital to the problem at hand (Cohen, 1995). As a result, one needs to use mathematical techniques that combine spatial/temporal content with spectral content (Hogan and Lakey, 2003).

One of the most fundamental and popular mathematical tools employed in signal pro- 
cessing is the Fourier transform. The Fourier transform, converts a signal from the time domain to the frequency domain by decomposing the respective signal into groups of waveforms (Qian, 2002). In essence the Fourier transform links time and frequency by breaking a time waveform into a group of frequencies. Hence, a given signal, through the prism of the Fourier transform has two major facets: time waveform and frequency spectrum (Qian, 2002).

In seismic signal analysis, there are several motivating factors for frequency or spectral analysis. First and foremost, spectral analysis of a waveform provides a certain degree of information about the source. Second, the relationship between the wave propagation through a given medium and frequency, is such that, waves with different frequencies propagate with different velocities, a phenomenon known as "dispersion". Furthermore, wave propagation is subject to medium and frequency dependent attenuation or loss of energy. In order to study wave propagation through a frequency dependent media, one needs to decompose a signal into different frequency components, analyse each frequency component and subsequently reconstruct the signal in order to obtain the resulting waveform, hence the need for the Fourier transform (Cohen, 1995). The third reason for signal decomposition relates to the representation of waveforms as superposition of sinusoids. Mathematically, a signal expanded in terms of sinusoids of different frequencies is given by (Cohen, 1995)

$$
s(t)=\frac{1}{2 \pi} \int_{-\infty}^{+\infty} S(\omega) e^{-i \omega t} d \omega,
$$

where $\omega$ represents the radial frequency. Clearly the signal is composed of the superposition of simple waveforms, $e^{-i \omega t}$ characterised by the radial frequency $\omega$ (Cohen, 1995). Hence, the Fourier transform provides greater insight and simplifies one's understanding of the waveform. Finally, based on the convolution theorem, the Fourier transform converts time consuming convolutions in one domain (e.g. time domain) into a simple point multiplications in the transformed domain (e.g. frequency domain).

However, the Fourier transform smears a signal's local behaviour by providing a global 
or average description of a signal's characteristic. Essentially the Fourier transform based methods are effective as long as the frequency content of a given signal do not change or evolve with time. Thus, the Fourier transform is a poorly suited method for analysing signals with rapid or slow frequency changes (Qian, 2002).

The shortcomings of the Fourier transform have resulted in the development of localised time frequency alternatives such as the short time Fourier transform or the wavelet transform. Instead of globally processing the entire signal, the short time Fourier transform (STFT), performs the Fourier transform on a block-by-block basis, describing how the spectrum of a signal evolves or changes with time (Qian, 2002). Hence, the STFT is a very well suited method for analysing the narrowband instantaneous frequency bandwidth (Qian, 2002).

Alternatively, the wavelet transform (closely related to multi-scale edge detection) compares a signal with a set of short waveforms or wavelets, such that each wavelet has a different time duration or scale (Qian, 2002). Additionally, an inverse correlation or relationship exists between the scale and frequency, such that the shorter the time duration or smaller the scale, the wider the frequency bandwidth and vice versa (Qian, 2002). Essentially, the wavelet transform decomposes the signals into fundamental building blocks localised in space and frequency, hence, providing a mathematical description for a signal's local behaviour. Furthermore due to dilation (mathematical terminology for fundamental wavelet stretching or compression), the wavelet exhibits an impulse-like behaviour, as it becomes narrower (Qian, 2002). Hence, the wavelet transform is a very powerful mathematical tool for detecting and analysing impulse type signals; non-stationary signals with jump discontinuities or local singularities .

\subsubsection{Seismic Attenuation and $Q$}

Traditionally, seismic wave propagation has been explained by the elastic wave equation modelled within an ideal elastic medium (Zhang, 2008). However in practical applications the elastic wave equation has been inadequate and in some instances an inaccurate description 
of seismic wave propagation in complex media. For example, propagating seismic waves within the earth experience absorption and irreversible conversion of energy into heat due to anisotropy and heterogeneity.

It is generally understood that due to absorption, the energy of seismic waves propagating through an anelastic medium would dissipate over a given distance. Thus, the absorptive properties of the medium distort the shape of the transient waveform(s), resulting in change of the corresponding amplitude and frequency content (Kjartansson, 1979; Zhang, 2008).

Seismic attenuation is generally categorised into scattering and intrinsic attenuation. Scattering attenuation distributes seismic wave energy within the medium in arbitrary directions and depends on the media's heterogeneity such that higher frequency components are lost due to destructive interference for scales of heterogeneity smaller than characteristic wavelengths (Mittet et al., 1995; Quan and Harris, 1997). Intrinsic attenuation, mainly caused by internal friction, refers to processes that convert energy into heat.

The overall effect of internal friction is described by the dimensionless quality factor $Q$ with studies concentrating either on the microscopic processes leading to attenuation, frequency dependence of $Q$ or developing and obtaining equations of motion for material with specific $Q$ based on the stress-strain relation (Aki and Richards, 2002). The dimensionless quantity $Q$ is typically defined by the following (Kjartansson, 1979; Aki and Richards, 2002; Lines et al., 2008),

- $Q=-\frac{2 \pi E}{\triangle E}$ where $E$ is the peak strain energy stored and $\triangle E$ represents the energy lost per cycle.

- $Q=\frac{4 \pi \omega}{\Delta \omega}$ where $\omega$ is the mean energy stored and $\triangle \omega$ is the mean energy loss per single cycle of sinusoidal deformation.

- $Q=\frac{\pi f}{v \alpha}$ where $\alpha$ denotes the absorption coefficient, $f$ and $v$ represent the temporal frequency and seismic velocity respectively. 
- $\frac{1}{Q}=\tan (\gamma)$ where $\gamma$ represents the phase angle between stress and strain.

It should be noted that the $Q$ definitions mentioned above are not equivalent.

\section{$1.3 \quad Q$ estimation methods}

Generally $Q$ estimation methods can be separated into time domain and frequency domain methods. Some of the most common techniques include the amplitude decay method, wavelet modelling, phase modelling and the spectral ratio method (Tonn, 1991).

\subsubsection{Amplitude decay method}

Considered to be one of the simplest methods, the $Q$ value is given by the following expression (Tonn, 1991)

$$
Q=\frac{\omega \triangle x}{2 c}\left\{\ln \frac{a\left(x_{1}\right)}{a\left(x_{2}\right)}\right\}^{-1},
$$

where $\omega$ represents the temporal frequency, $a\left(x_{1}\right)$ and $a\left(x_{2}\right)$ represent the amplitudes corresponding to receivers located at $x_{1}$ and $x_{2}$ respectively. It should be noted that this method requires true amplitude recordings (Tonn, 1991).

\subsubsection{Wavelet modelling method}

Introduced by Jansen et al. (1985), the wavelet modelling method utilises the travel-time difference and dispersion relation, in order to approximate an optimum $Q$ value to an observed signal at depth $x_{2}$ by synthetically modifying a reference signal at depth $x_{1}$ through varying $Q$ values (Tonn, 1991). Typically, one could compare the modelled signal with the observed signal via the $L_{1}$ norm (difference between the amplitude of the wavelets) or the $L_{2}$ norm (difference of the squares) (Tonn, 1991). 


\subsubsection{Phase modelling method}

Analogous to the wavelet modelling method, by utilising the dispersion relation and synthetically modifying the instantaneous phases of a reference signal (by varying $Q$ values), one could obtain an optimum $Q$ approximation to the observed signal. However, contrary to the wavelet modelling method, one can only apply the $L_{1}$ norm in order to compare the modelled signal with the observed signal (in this case the $L_{2}$ has no significant physical justification) (Tonn, 1991).

\subsubsection{Spectral ratio method}

Arguably the one of best known methods for $Q$ computation, the spectral ratio applies changes in spectra at varying depth values. By computing the slope, one could obtain the corresponding $Q$ value from the following expression (Haase and Stewart, 2004)

$$
\ln \left[\frac{\left|A_{2}(\omega)\right|}{\left|A_{1}(\omega)\right|}\right]=(\text { const. })-\omega \frac{\triangle x}{2 c Q},
$$

where $A_{1}(\omega)$ and $A_{2}(\omega)$ represent the spectral amplitudes at depth values $x_{1}$ and $x_{2}$ respectively and $\triangle x$ corresponds to the depth difference.

Over the past several decades additional $Q$ estimation methods, such as the matching technique, risetime method, frequency modelling and analytic signal method have been proposed, applied in industry and extensively discussed in literature ${ }^{1}$. However, the noted $Q$ estimation methods are not equivalent (the thesis objective is to estimate and compare $Q$ values to traditional methods such as the steepest descent).

\subsection{Seismic signal smoothness \& Lipschitz regularity}

A seismic pulse that undergoes attenuation can be modelled as a delta function characterised by an amplitude $A$, order of singularity with local regularity $\alpha$ and smoothness convolved

\footnotetext{
${ }^{1}$ For in depth description, the following is recommend to readers: (Tonn, 1991).
} 
with a Gaussian of variance $\sigma^{2}$ (Innanen, 2003). The objective is to utilise the effects of $Q$ and subsequent local regularity on a seismic signal as a framework for a potential mathematical mapping or relation between the two parameters. From Kjartansson (1979) and Strick (1970) the time-domain impulse response is given by

$$
b(t)= \begin{cases}\psi(Q) t_{s}^{-\frac{1}{2}} t_{s}^{-\frac{1}{2 \gamma}}, & t \geq 0 \\ 0, & t<0\end{cases}
$$

where $t_{s}=t\left(\frac{\triangle z}{c_{0} \omega_{r}^{-\gamma}}\right)^{\frac{1}{(1-\gamma)}}, \gamma=\frac{1}{\pi} \tan ^{-1}\left(\frac{1}{Q}\right), c_{0}$ represents the wave velocity and $\psi(Q)$ represents a combination of terms altering the casual response and smoothness (Innanen, 2003). Since the amplitude and smoothing do not depend on the Lipschitz exponent $\alpha$, one could re-write the impulse response as

$$
b(t) \approx\left\{\begin{array}{ll}
\psi_{0} t_{s}^{-\frac{1}{2}} t_{s}^{-\frac{1}{2 \gamma}}, & t \geq 0 \\
0, & t<0
\end{array},\right.
$$

by defining $\psi(Q) \equiv \psi_{0}$ (Innanen, 2003). As absorption decreases with $Q \rightarrow \infty$, then $\gamma \rightarrow 0$ and the combined exponents $-\frac{\gamma+1}{2 \gamma} \rightarrow-\infty$. Hence, for large $Q$ values, the impulse response displays "delta type" behaviour. As a result, the overall regularity $\alpha$, will remain within a range of -1 (Innanen, 2003). Conversely, by replacing the non-linear term $\gamma=\frac{1}{\pi} \tan ^{-1}\left(\frac{1}{Q}\right)$ with its large $Q$ approximation $\gamma=\frac{1}{\pi Q}$ and letting $Q \rightarrow 0$ (increasing absorption), the impulse response becomes "step like", hence the Lipschitz regularity $\alpha$ tends towards 0 . In case of seismic events, the main challenge relates to detecting and mathematically describing the potential mapping for Lipschitz values ranging from -1 to 0 .

\subsection{Vertical seismic profile (VSP) data}

A vertical seismic profile (VSP) refers to seismic data sets recorded with vertical or nearvertical distribution of seismic sensors or geophones (Simman, 2007). During the past two 
decades, a VSP has been widely used in a variety of geological provinces in order to improve and assist geophysical data processing and geological interpretation (Kennett et al., 1980)

Although a VSP requires new processing requirements (because of vertical distribution of geophones in direction of wave propagation), it can typically provide valuable information, due to the fact that part of the measurement and observation takes pace within the earth (Balch and Myung, 1984). For example, seismologists can observe the formation of reflections (primaries, multiples and converted waves) and source pulse distortion and attenuation as it propagates and progresses through the earth (Balch and Myung, 1984). Figure 1.1 illustrates the effects of absorption on a seismic pulse as it propagates through the medium. Clearly, the seismic pulse experiences loss of amplitude and certain degree of smoothness (pulse broadening) as it propagates through the absorptive medium.

Furthermore, one could measure acoustic properties of rocks and infer relationships between acoustic properties and lithology, porosity, permeability and fluid content (Balch and Myung, 1984). Furthermore, at any point in the subsurface, a VSP allows separation and independent study and analysis of downgoing and upgoing travelling waveforms (Kennett et al., 1980).

Generally, VSP data enables access to direct arrivals corresponding to receivers of geophones located at various depth values, hence an ideal data set for studying and analysing attenuation. 


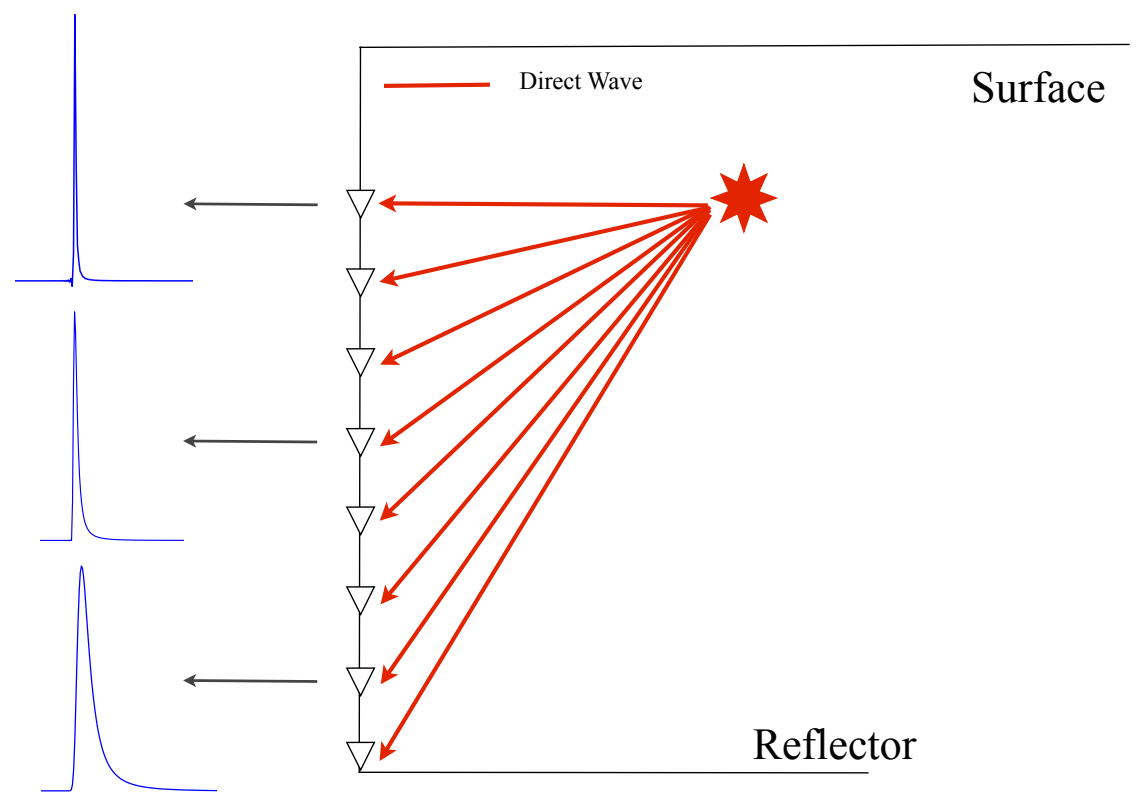

Figure 1.1: Illustration of VSP experiment and the effects of absorption on a seismic pulse. The seismic pulse undergoes the process of smoothness (pulse broadening) and loss of amplitude as it propagates through the absorptive medium.

\subsection{Thesis objective}

The underlying problem and ultimate objective is to provide a mathematical expression that describes the relationship between the local signal properties (local regularity and smoothness), and the seismic quality factor, $Q$. Essentially, the solution to the problem consists of the following three steps,

1. Assessing the stability of the linear and non-linear Mallat-Hwang-Zhong signal model and subsequently verifying the associated properties of the Lipschitz exponent.

2. Creating a synthetic VSP model with varying depth and $Q$ values and subsequently applying the continuous wavelet transform on the corresponding direct arrival in order to measure the associated regularity and smoothness values, 
hence provide a mathematical mapping between absorption and local signal properties.

3. Applying the obtained results from synthetic analysis to VSP field data in order to estimate the corresponding $Q$ values.

\subsection{Thesis outline}

The thesis is organised as follows:

- Chapter II consists of a theoretical description of time-frequency methods, the continuous wavelet transform and its corresponding properties. Furthermore, a detailed description of the relationship between the wavelet transform modulus maxima, Lipschitz exponent $\alpha$ and mathematical methods for estimating the Lipschitz exponent from the Mallta-Hwang signal model is given in this chapter.

- Chapter III provides a mathematical model for estimating the regularity and smoothness of "delta type" and "step like" functions along with their corresponding Lipschitz exponent. Additionally, the effects and limitations of the model for closely spaced events is discussed.

- Chapter IV provides a synthetic case study of $Q$ estimation from VSP data and the mathematical relationship between $Q$. local regularity and smoothness based on Mallat-Hwang-Zhang model parameters.

- In Chapter V the results from the previous chapter are applied to the Ross Lake VSP field data in order to estimate and compare the results to previously estimated $Q$ values. 
- Finally, Chapter VI concludes the thesis with an overview of the results and recommendations for future work. 


\section{Chapter 2}

\section{Continuous wavelet transforms and Lipschitz regularity}

Compared to the existing time-frequency transformations, the continuous wavelet transform provides a mathematical description of a function's local behaviour. The local regularity or behaviour of a seismic event is determined by the wavelet transform modulus maxima and the associated Lipschitz exponent. As a means of classifying regularities of a seismic signal and estimating the associated Lipschitz exponent, a linear and non-linear Mallat-Hwang-Zhong signal model based on the wavelet theory is reviewed.

For certain kinds of signal events (impulse type events), the linear model can be applied in order to determine the associated Lipschitz regularity. However, for band-limited signal events with some degree of smoothness a more complex non-linear model has to be applied. The non-linear signal model includes three parameters (as opposed to two for the linear model), in order to fully reflect and characterise a propagating seismic pulse as it experiences smoothness and loss of amplitude due to absorption. Hence, in order to estimate the associated parameters (mainly the local regularity and smoothness), one would need to apply the least squares method or a non-linear optimisation method such as the steepest descent.

\subsection{Time-frequency methods}

Over the past two centuries the Fourier transform, ubiquitous in science and engineering, has been applied in solving a wide range of problems encompassing the fields of signal processing, probability theory and quantum mechanics to name a few. In essence, the Fourier transform decomposes a function into sinusoidal waveforms, hence providing a mapping between the space/time and frequency domain (Mallat, 2009; Brigham, 1988).

Despite its versatility and efficiency in analysing periodic functions or stationary signals, 
the Fourier transform is ill-suited and inefficient in representing transient phenomena, as it fails to provide sufficient information in regards to the evolution of frequency content in time or local properties of the frequency content, since it integrates a given function $f(t)$ over all time (Daubechies, 1992; Kaiser, 1994; Qian, 2002). In other words, the global property of the Fourier transform prevents local analysis of $f(t)$ from $\widehat{f}(\omega)$ (Mallat, 2009).

In order to overcome the limitation(s) associated with the Fourier transform and provide a local description of a given function, Dennis Gabor (1946) introduced an analysis window of fixed size in order to perform a "time localised" Fourier transform (Gao and Yan, 2011; Okamura, 2011). In essence this method, referred to as the short-time Fourier transform or the windowed Fourier transform, maps a desired signal into a two dimensional function of time and frequency by cutting a given function $f(t)$ into blocks and subsequently performing the Fourier transform on a block by block basis which in turn provides information in regards to the signal's frequency content or behaviour during the time frame covered by the corresponding window (illustrated in Figure 2.1) (Gao and Yan, 2011; Qian, 2002; Kaiser, 1994). The short-time Fourier transform can be described by the following mathematical expression,

$$
\operatorname{STFT}\{f(t)\}(\tau, \omega)=\int_{-\infty}^{+\infty} f(t) g(t-\tau) \mathbf{e}^{\mathrm{i} \omega t} d t,
$$

where $f(t)$ is an arbitrary signal in $\mathbf{L}^{2}(\mathbb{R})$ and $g(t)$ is the fixed window designed to localise signals in time (Daubechies, 1992; Gao and Yan, 2011). Equation 2.1 represents a convolution between the signal $f(t)$ and the time-shifted, frequency-modulated window $g(t)$. Various types of window functions have been developed specifically tailored for a particular application, from the Gaussian window designed for analysing transient signals to Hamming-Hann windows applicable to random, narrowband signals (Gao and Yan, 2011).

Despite the benefits of time-frequency localisation provided by the short-time Fourier transform, an inherent drawback relates to the inflexible size of the window function. For a small window, low frequencies are too large to be represented accurately. Additionally, 
one would have to use large windows for high frequencies which would result in loss of information for brief or abrupt changes in the corresponding interval (Qian, 2002). As a result, due to the rigid dimensional nature of the window, the short-time Fourier technique does not guarantee and at times inhibits effective signal decomposition (Gao and Yan, 2011; Qian, 2002). In order to overcome the shortcomings associated with the short-time Fourier transform, alternative techniques such as the wavelet transform has been proposed as a means of analysing non-stationary signals. 

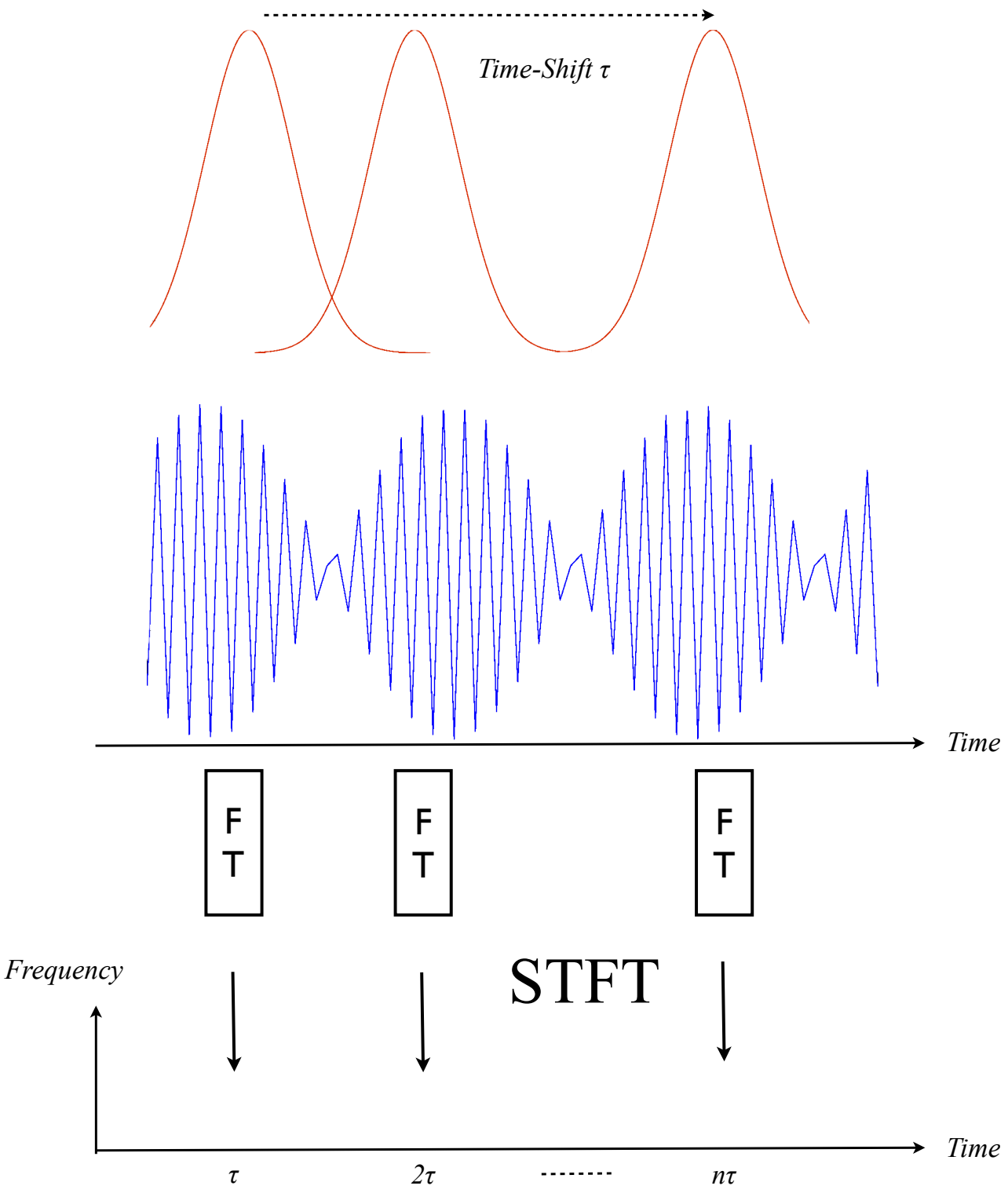

Figure 2.1: Visual description of the short-time Fourier transforms. The short-time Fourier transform performs the Fourier transform on a block by block basis by multiplying the signal with a windowed function (in this case a Gaussian window) and subsequently computing the Fourier transform of the product. Moving the windowed function and repeating the process provides an idea about the evolving frequency content of a given signal over time (Qian, 2002). 


\subsubsection{Wavelet tranforms}

Historically, the first reference to wavelet(s) dates back to the early twentieth century when Alfred Haar (1910) constructed the following function,

$$
\psi(t)= \begin{cases}1 & 0 \leq t<\frac{1}{2} \\ -1 & \frac{1}{2} \leq t<1 \\ 0 & \text { otherwise }\end{cases}
$$

such that the dilations and translations of $\psi(t)$ generates an orthonormal basis in the $\mathbf{L}^{2}(\mathbb{R})$ space (Gao and Yan, 2011; Mallat, 2009). However, the theoretical formation and foundation of the wavelet transform is for the most part attributed to Jean Morlet and Alex Grossmann (1983) for proposing a technique for scaling and shifting the analysis window and additionally introducing the notion that a signal can be transformed into a wavelet and back into its original structure without loss of information (Gao and Yan, 2011; Mallat, 2009; Qian, 2002).

The wavelet transform divides a given function or signal into different scale components, and assigns a frequency range to each scale component by utilising a scalable modulated window, that calculates the spectrum at every position and shifts the scalable window along the signal, hence providing a time-scale representation of a given function or signal (Qian, 2002). Figure 2.2 illustrates the difference between the short-time Fourier transform and the wavelet transform. In the short-time Fourier transform, the size of the windowed function is fixed regardless of the number of oscillations, whereas a wavelet adjusts the width, essentially keeping the number of oscillations constant (Qian, 2002).

Mathematically, for a given function $f(t)$ the continuous wavelet transform is given by the following relation,

$$
\mathbf{W} f(s, \tau)=\frac{1}{\sqrt{s}} \int_{-\infty}^{+\infty} f(t) \psi^{*}\left(\frac{t-\tau}{s}\right) d t
$$

where $s$ represents the scaling factor inversely proportional to the frequency, $\tau$ represents translation along the time axis and $\psi^{*}($.$) denotes the complex conjugate of the "mother$ 
wavelet" $\psi(t)$ (Gao and Yan, 2011; Qian, 2002). Mathematically, dilation (scaling) and translation (time-shifting) of the mother wavelet produces a family of wavelets. Furthermore, a wavelet must satisfy the following "admissibility condition" (Daubechies, 1992; Gao and Yan, 2011; Qian, 2002),

$$
\int_{-\infty}^{+\infty} \frac{|\widehat{\psi}(\omega)|^{2}}{|\omega|} d \omega<\infty
$$

The admissibility condition implies that,

$$
|\widehat{\psi}(0)|_{\omega=0}=0
$$

hence

$$
\int_{-\infty}^{+\infty} \psi(t) d t=0
$$

For a wavelet satisfying the admissibility condition, one could reconstruct a given signal $f(t)$ by applying the corresponding inverse continuous wavelet transform. Mathematically, the inverse continuous wavelet transform is given by the following expression (Gao and Yan, 2011; Qian, 2002; Daubechies, 1992),

$$
f(t)=\frac{1}{C_{\psi}} \int_{0}^{+\infty} \int_{-\infty}^{+\infty} \frac{1}{s^{2}} \mathbf{W} f(s, \tau) \frac{1}{\sqrt{s}} \psi\left(\frac{t-\tau}{s}\right) d s d \tau
$$

where $C_{\psi}=\int_{-\infty}^{+\infty} \frac{|\widehat{\psi}(\omega)|^{2}}{|\omega|}<\infty$.

Based on Equation 2.6, one could make a distinction between functions that resemble a wavelet, such as scaling functions, and an actual wavelet by ensuring that the function integrates to zero (Figure 2.3). For practical applications and in order to implement a fast numerical algorithm one would have to sample the continuous wavelet algorithm on a dyadic grid, that is $s=2^{j}$, for $j \in \mathbb{Z}$, where it has been proven that the wavelet transform on a dyadic grid is complete and stable (Mallat and Zhong, 1992). 

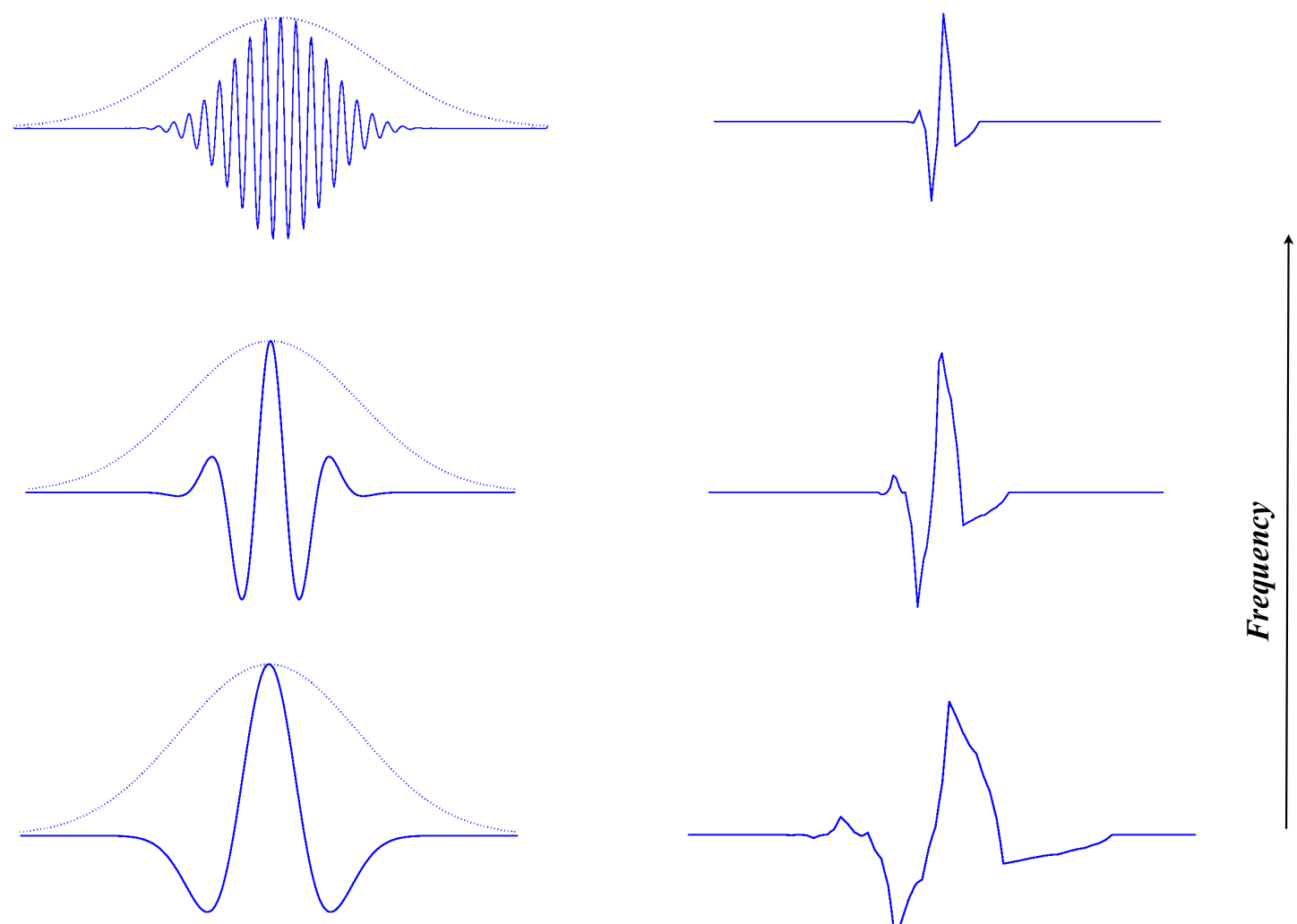

STFT

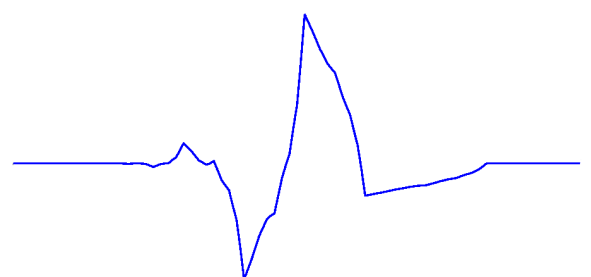

Wavelet

Figure 2.2: The wavelet (right side) varies the width while keeping the number of oscillations constant. The short-time Fourier transform (left side) has a fixed window size independent of oscillations. 


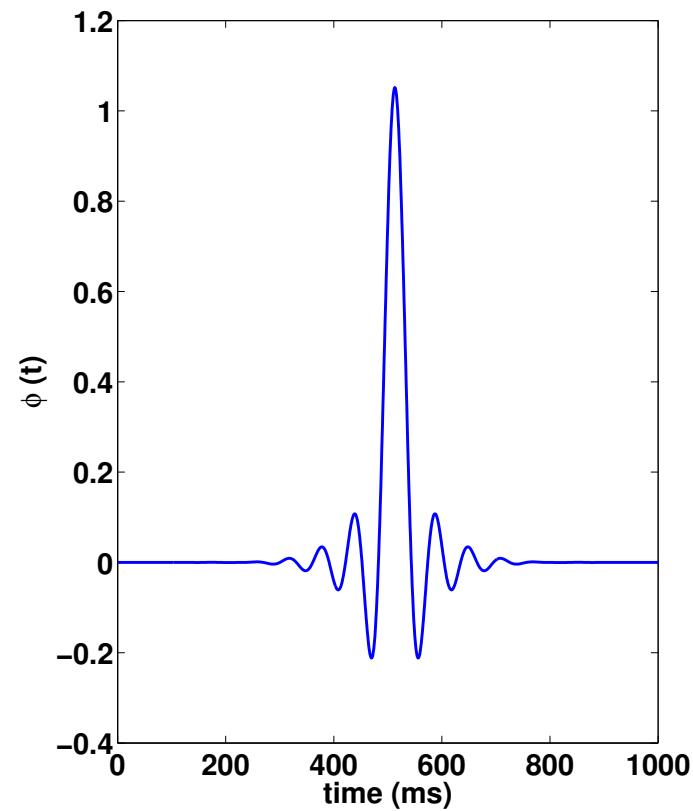

(a)

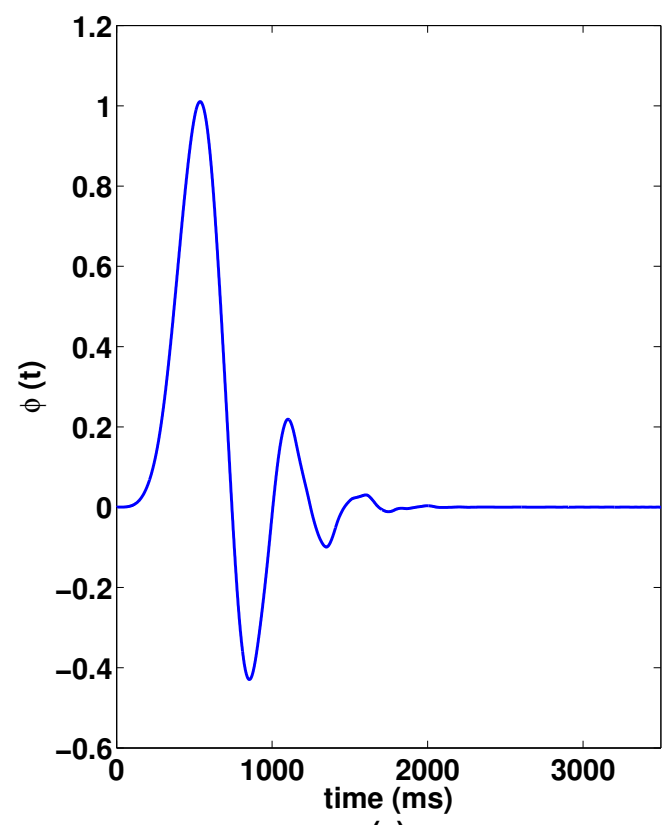

(c)

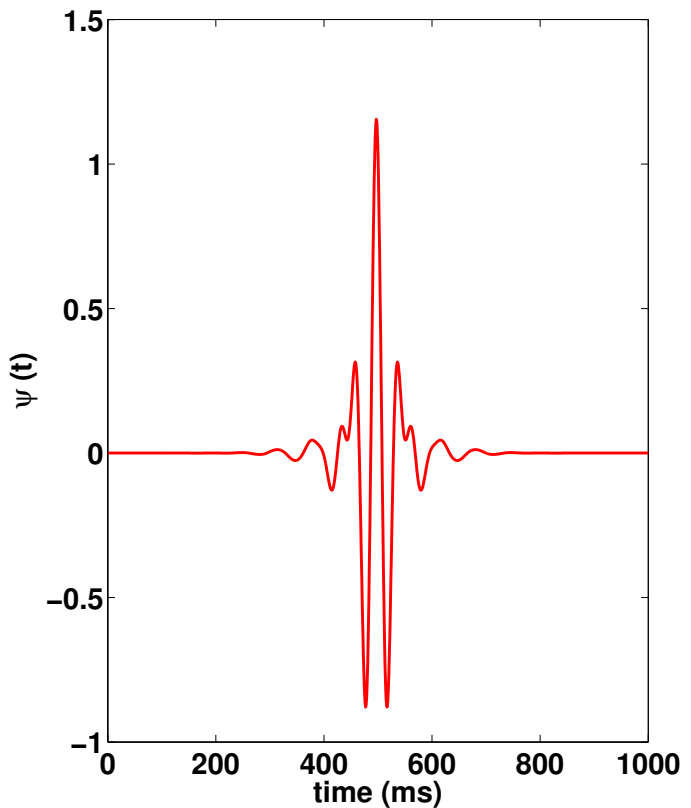

(b)

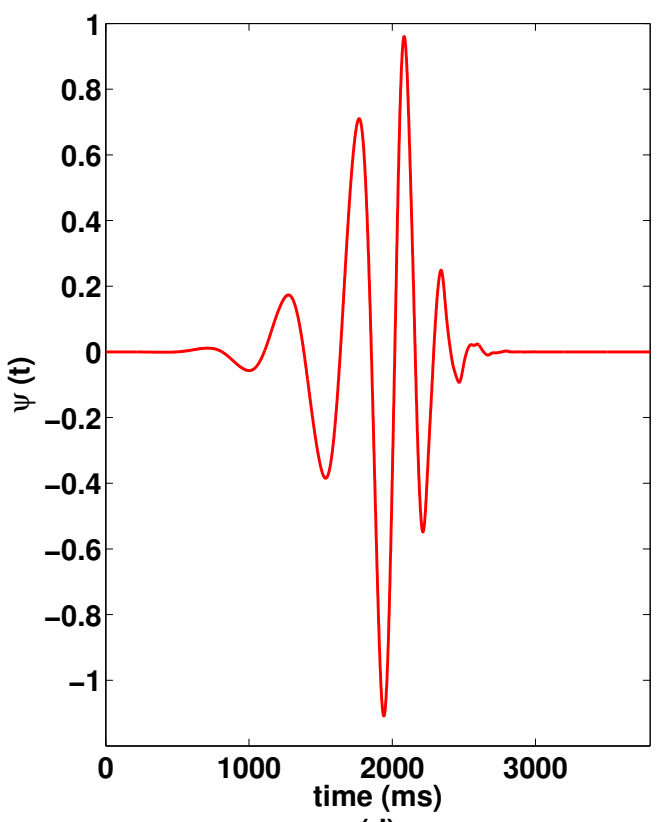

(d)

Figure 2.3: A wavelet function (right side) as opposed to scaling function (left side) must integrate to zero in the time domain. (a) Meyer scaling function (b) Meyer wavelet (c) Daubeschies "db8" scaling function (d) Daubeschies "db8" wavelet. 


\subsection{Properties of the continuous wavelet transform (CWT)}

By definition, the continuous wavelet transform is a linear operator characterised by the following properties (Gao and Yan, 2011; Farge, 1992; Sheng, 2000) ${ }^{1}$.

\subsubsection{Superposition}

For a given function $f(t)$, the continuous wavelet transform satisfies the following relation

$$
\mathbf{W}\left(f_{1}+f_{2}\right)(s, \tau)=\mathbf{W} f_{1}(s, \tau)+\mathbf{W} f_{2}(s, \tau) .
$$

\subsubsection{Translation and dilation}

The continuous wavelet transform is covariant under any translation or dilation, that is, for $f\left(t-t_{0}\right)$ and $f\left(\frac{t}{a}\right)$ the continuous wavelet transform given by

$$
\left(\mathbf{W} T_{t_{0}} f\right)(s, \tau)=(\mathbf{W} f)\left(s, \tau-t_{0}\right)
$$

and

$$
\left(\mathbf{W} D_{a} f\right)(s, \tau)=\sqrt{a}(\mathbf{W} f)\left(\frac{s}{a}, \frac{\tau}{a}\right)
$$

where

$$
\left(T_{t_{0}} f\right)(t)=f\left(t-t_{0}\right)
$$

and

$$
\left(D_{a} f\right)(t)=f\left(\frac{t}{a}\right)
$$

\subsubsection{Local regularity}

An important property associated with the continuous wavelet transform is the ability to characterise local regularities by smoothing a given signal $f$ and detecting points of sharp variation at various scales $s$. The local regularity of a function $f$ is often measured by the corresponding Lipschitz or Hölder exponent (Mallat and Zhong, 1992; Hong et al., 2002).

\footnotetext{
${ }^{1}$ For proof the following reading is recommended: (Gao and Yan, 2011)
} 


\subsection{The Lipschitz exponent}

The Lipschitz exponent, a generalised measure of a function's differentiability is defined in the frequency domain by the following relation (Daubechies, 1992),

$$
\int_{-\infty}^{+\infty}|\widehat{f}(\omega)|(1+|\omega|)^{\alpha} d \omega<+\infty .
$$

Based on Equation 2.13, the Lipschitz exponent $\alpha$, corresponds to the decay of the Fourier coefficients with increasing frequency and equal to the supremum of all $\alpha$ values satisfying Equation 2.13 (Innanen, 2003). However, Equation 2.13 is not well adapted for measuring local regularity of a function at a specific point since it only provides a global regularity condition. As a result one would have to apply wavelet analysis in order to gain information in regards to local regularity of a function. Based on the continuous wavelet transform, a function $f(x)$ is said to be uniformly Lipschitz $\alpha$ over $[a, b]$ if and only if there exists a constant $A>0$ such that the wavelet transform satisfies the following (Mallat and Zhong, 1992; Innanen, 2003),

$$
\left|\mathbf{W}_{s} f(x)\right| \leq A s^{\alpha}
$$

where $|\mathbf{W} f(x)|$ is the modulus maxima of the function $f(x)$ at various scales $s=2^{j}$ for $j \varepsilon \mathbb{Z}$. It should be noted that the Lipschitz exponent $\alpha$, is assumed to be constant over the interval which the signal is analysed. Equation 2.14 suggests that the evolution of the modulus of the wavelet coefficients across the scale depends on the local Lipschitz regularity of the desired function (Innanen, 2003). Thus, based on the following properties associated with the Lipschitz exponent, a distinction could be made between singular and differentiable function (Mallat and Zhong, 1992; Hermann, 1997)

- For a continuous and differentiable function, $f(x)$, the associated Lipschitz exponent $\alpha$, is equal to or greater than 1 .

- A function $f(x)$ is singular if the associated Lipschitz exponent, $\alpha$, is less than 1. 
- The Lipschitz regularity of a delta function is equal to -1 , since its associated modulus maxima decreases with scale.

- If $f(x)$ is Lipschitz $\alpha$, then its integral $g(x)$ has an associated Lipschitz exponent equal to $\alpha+1$.
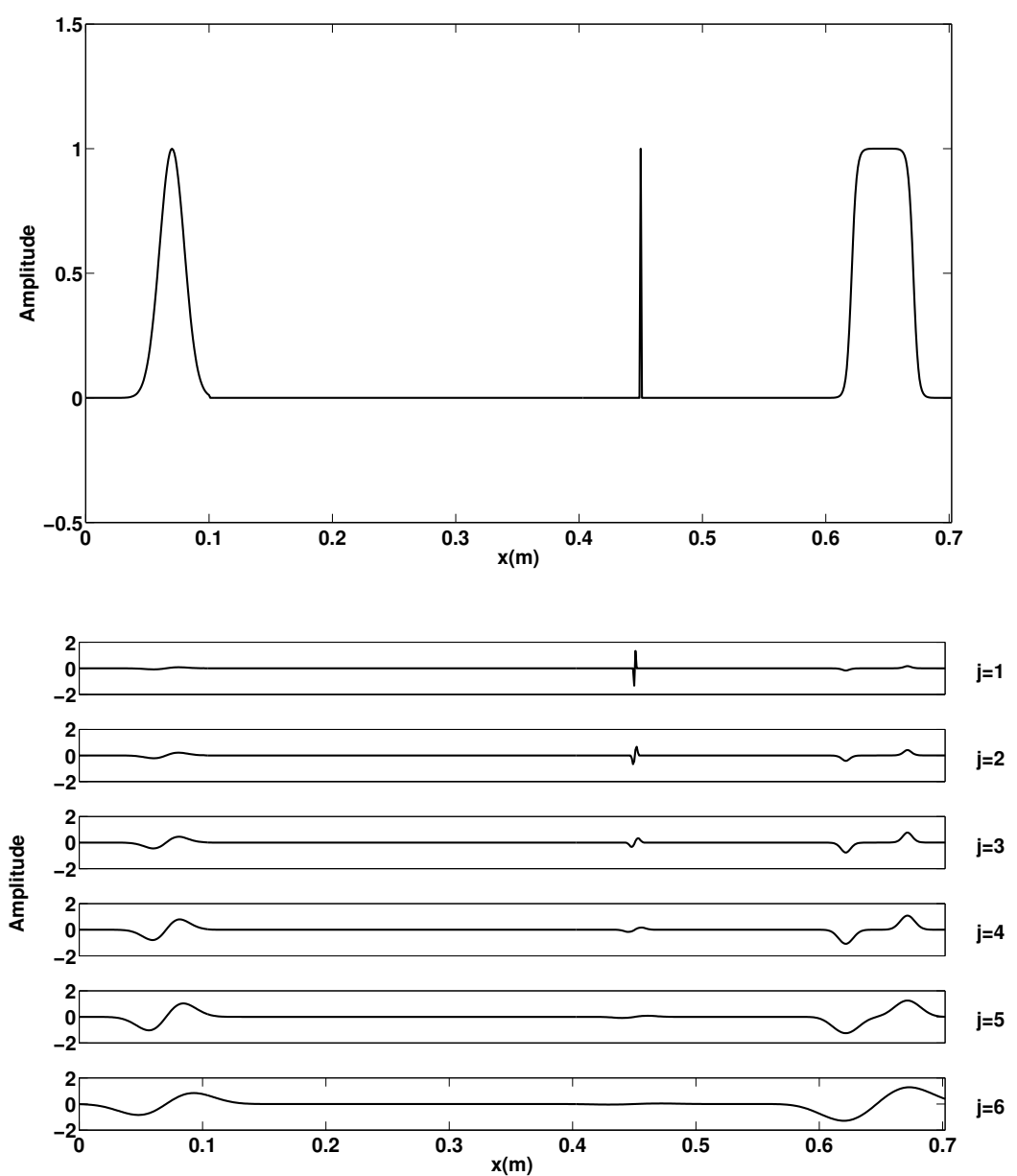

Figure 2.4: Original signal and corresponding continuous wavelet transform at scales, $s=2^{j}$.

\subsection{Estimating Lipschitz regularity}

In order to estimate the Lipschitz exponent $\alpha$ from the data, one could linearise Equation 2.14 by taking the logarithms in order to obtain the following relation,

$$
\log _{2}\left|\mathbf{W}_{s} f(x)\right| \leq \log _{2}|A|+\alpha \log _{2}(s)
$$


For $s=2^{j}$, equation 2.15 reduces to the following expression,

$$
\log _{2}\left|\mathbf{W}_{s} f(x)\right| \leq \log _{2}|A|+\alpha j
$$

Finding the slope and intercept of the relation given above (2.16), yields an estimate for $\alpha$ and $A$. Although linearising Equation 2.14 simplifies the estimation of $\alpha$, nevertheless this procedure requires a certain degree of caution since it involves scaling the errors associated with the numerical estimation of the modulus maxima. Additionally one could estimate $\alpha$ and $A$ by posing Equation 2.16 as an optimisation problem. Forming the objective function, one would obtain the following expression

$$
\phi(\alpha, A)=\sum_{i, j=1}^{n}\left[\log _{2}\left|a_{i}\right|-\left(\log _{2}|A|+\alpha \log _{2}\left(s_{j}\right)\right)\right]^{2},
$$

where $a_{i}=\left|\mathbf{W}_{s_{j}} f(x)\right|$ and $s_{j}=2^{j}$ for $i, j=1,2,3, \ldots, n$. Minimising the objective function provides the following system of equations (Burden and Douglas, 2005)

$$
\left(\begin{array}{c}
\log _{2}|A| \\
\alpha
\end{array}\right)=\left(\begin{array}{cc}
n & \sum_{j=1}^{n} \log _{2}\left(s_{j}\right) \\
\sum_{j=1}^{n} \log _{2}\left(s_{j}\right) & \sum_{j=1}^{n}\left[\log _{2}\left(s_{j}\right)\right]^{2}
\end{array}\right)^{-1}\left(\begin{array}{c}
\sum_{i=1}^{n} \log _{2}\left(a_{i}\right) \\
\sum_{j=1}^{n} \log _{2}\left(s_{j}\right) \cdot \sum_{i=1}^{n} \log _{2}\left(a_{i}\right)
\end{array}\right) .
$$

\subsubsection{The Mallat-Hwang-Zhong signal model}

In seismic signal analysis, our main interest rests on estimating the Lipschitz values ranging from $-1 \leq \alpha \leq 0$. Thus it is preferable to use a wavelet with a single vanishing moment, such as the first derivative of a Gaussian function. A wavelet $\psi(x)$ is said to have $n$ vanishing moments if for $k<n$ it satisfies (Mallat and Hwang, 1992),

$$
\int_{-\infty}^{+\infty} x^{k} \psi(x) d x=0
$$

Furthermore, letting $\psi(x)=\frac{d \theta(x)}{d x}$, where $\theta(x)$ is a Gaussian function, and taking the continuous wavelet transform of $f$ we obtain the following expression (Mallat and Hwang, 1992)

$$
\mathbf{W}_{s} f(x)=f *\left(s \frac{d \theta_{s}}{d x}\right)(x)=s \frac{d}{d x}\left(f * \theta_{s}\right)(x)
$$


Based on equation 2.20, the local extrema of $\mathbf{W}_{s} f(x)$ corresponds to the inflection points of $f * \theta_{s}(x)$. Hence, the inflection points or points of sharp variation corresponding to $f * \theta_{s}(x)$ could be detected by estimating the local extrema of $\left|\mathbf{W}_{s} f(x)\right|$ (Mallat and Zhong, 1992).

Due to absorption and loss of energy, a pulse undergoes a degree of smoothing, thus gradually obtaining spectral characteristics of a Gaussian. To model and measure the smoothness of the signal variation, a delta function $h(x)$ is convolved with a Gaussian of variance $\sigma^{2}$ (Mallat and Zhong, 1992),

$$
f(x)=h(x) * g_{\sigma}(x)
$$

where $g_{\sigma}(x)=\frac{1}{\sqrt{2 \pi} \sigma} \exp \left(\frac{-x^{2}}{2 \sigma^{2}}\right)$. The continuous wavelet transform of 2.21 is given by the following

$$
\mathbf{W}_{s} f(x)=2^{j} \frac{d}{d x}\left(h * \theta_{s_{0}}\right)(x)=\frac{2^{j}}{s_{0}} \mathbf{W}_{s_{0}} h(x)
$$

where $\theta_{s_{0}}=g_{\sigma}(x) * \theta_{s}, s_{0}=\sqrt{2^{2 j}+\sigma^{2}}$ and $s=2^{j}$. As a result, the modulus maxima and Lipschitz regularity is given by the following relation (Mallat and Zhong, 1992; Mallat and Hwang, 1992),

$$
\left|\mathbf{W}_{s_{0}} h(x)\right|=\frac{s_{0}}{s}\left|\mathbf{W}_{s_{0}} f(x)\right| \leq A s_{0}^{\alpha}
$$

or

$$
\left|\mathbf{W}_{s_{0}} f(x)\right| \leq s A s_{0}^{\alpha-1}
$$

Taking the logarithm of 2.24 provides the following expression

$$
\log _{2}\left|a_{i}\right| \leq \log _{2}|A|+j+\frac{\alpha-1}{2} \log _{2}\left(\sigma^{2}+s^{2}\right)
$$

where $\left|a_{i}\right|=\left|\mathbf{W}_{s_{0}} f(x)\right|$.

In comparison to the linear model given by 2.16 , the new model is non-linear and requires minimisation of the following objective function,

$$
\phi(A, s, \sigma)=\sum_{i, j=1}^{n}\left[\log _{2}\left|a_{i}\right|-\log _{2}|A|-j-\frac{\alpha-1}{2} \log _{2}\left(\sigma^{2}+2^{2 j}\right)\right]^{2} .
$$


Evidently, the new model incorporates the linear model as the value of $\sigma$ decreases. Taking the limit of 2.25 as $\sigma \rightarrow 0$, one obtains the expression given by 2.15 ,

$$
\begin{gathered}
\lim _{\sigma \rightarrow 0} \phi(A, s, \sigma)=\lim _{\sigma \rightarrow 0}\left(\sum_{i, j=1}^{n}\left[\log _{2}\left|a_{i}\right|-\log _{2}|A|-j-\frac{\alpha-1}{2} \log _{2}\left(\sigma^{2}+2^{2 j}\right)\right]^{2}\right) \\
=\sum_{i, j=1}^{n}\left[\log _{2}\left|a_{i}\right|-\left(\log _{2}|A|+\alpha j\right)\right]^{2}
\end{gathered}
$$

Minimising the objective function given by 2.24 requires the least squares method or a nonlinear optimisation method such as the steepest descent.

\subsubsection{Implementation of the MHZ model: steepest descent}

Initially proposed by Cauchy (1847), the steepest descent also known as the gradient descent, determines a local minimum for a non-linear multivariate function by applying directional line search proportional to the negative of the gradient (Burden and Douglas, 2005; Meza, 2010; Wang, 2008). For a function defined by

$$
g\left(x_{1}, x_{2}, \ldots, x_{n}\right)=\sum_{i=1}^{n}\left[f_{i}\left(x_{1}, x_{2}, \ldots, x_{n}\right)\right]^{2},
$$

the following non-linear system of equations

$$
\begin{gathered}
f_{1}\left(x_{1}, x_{2}, \ldots, x_{n}\right)=0 \\
f_{2}\left(x_{1}, x_{2}, \ldots, x_{n}\right)=0 \\
\vdots \\
f_{n}\left(x_{1}, x_{2}, \ldots, x_{n}\right)=0,
\end{gathered}
$$

has a solution at $\mathbf{x}=\left(x_{1}, x_{2}, \ldots, x_{n}\right)^{T}$ when $g$ has a minimum value of 0 . To reduce $g(\mathbf{x})$ to its minimum value, one needs to (Burden and Douglas, 2005):

1. Evaluate $g$ at an initial guess/approximation $\mathbf{x}^{(\mathbf{0})}=\left(x_{1}^{(0)}, x_{2}^{(0)}, \ldots, x_{n}^{(0)}\right)^{T}$.

2. Determine a descent direction from $\mathbf{x}^{(\mathbf{0})}$.

3. Proceed an appropriate amount in the direction that decreases the value of $g$ and assign a new value $\mathbf{x}^{(\mathbf{1})}$. 
4. Update by repeating steps 1 through 3 until convergence is achieved.

An appropriate choice for $\mathbf{x}^{(\mathbf{1})}$ is given by the following relation,

$$
\mathbf{x}^{(1)}=\mathbf{x}^{(0)}-\lambda \nabla g\left(\mathbf{x}^{(0)}\right)
$$

where

$$
\nabla g(\mathbf{x})=\left(\frac{\partial g}{\partial x_{1}}(\mathbf{x}), \frac{\partial g}{\partial x_{2}}(\mathbf{x}), \ldots, \frac{\partial g}{\partial x_{n}}(\mathbf{x})\right)^{T}
$$

for $\lambda>0$. In order to assign a value for $\lambda$, such that $g\left(\mathbf{x}^{(1)}\right)<g\left(\mathbf{x}^{(0)}\right)$, one needs to proceed with one of the following steps (Burden and Douglas, 2005)

1. Differentiate $h(\alpha)=g\left(\mathbf{x}^{(0)}-\alpha \nabla g\left(\mathbf{x}^{(0)}\right)\right)$ and subsequently find the root value in order to determine the critical point(s) of $h$.

2. Choose $\alpha_{1}<\alpha_{2}<\alpha_{3}$, such that a quadratic polynomial $p(x)$ interpolates $h$ at $\alpha_{1}, \alpha_{2}$ and $\alpha_{3}$. Subsequently, use $p(\alpha)$, a minimum in $\left[\alpha_{1}, \alpha_{3}\right]$, to approximate the minimum value of $h(\alpha)$.

Compared to alternative optimisation methods such as the quasi-Newton or the conjugate gradient method, the steepest descent method is relatively simple to implement and requires lower storage, $O(n)$. However, a major drawback relates to the rate of convergence which is generally lower than the quasi-Newton or the conjugate gradient method. 


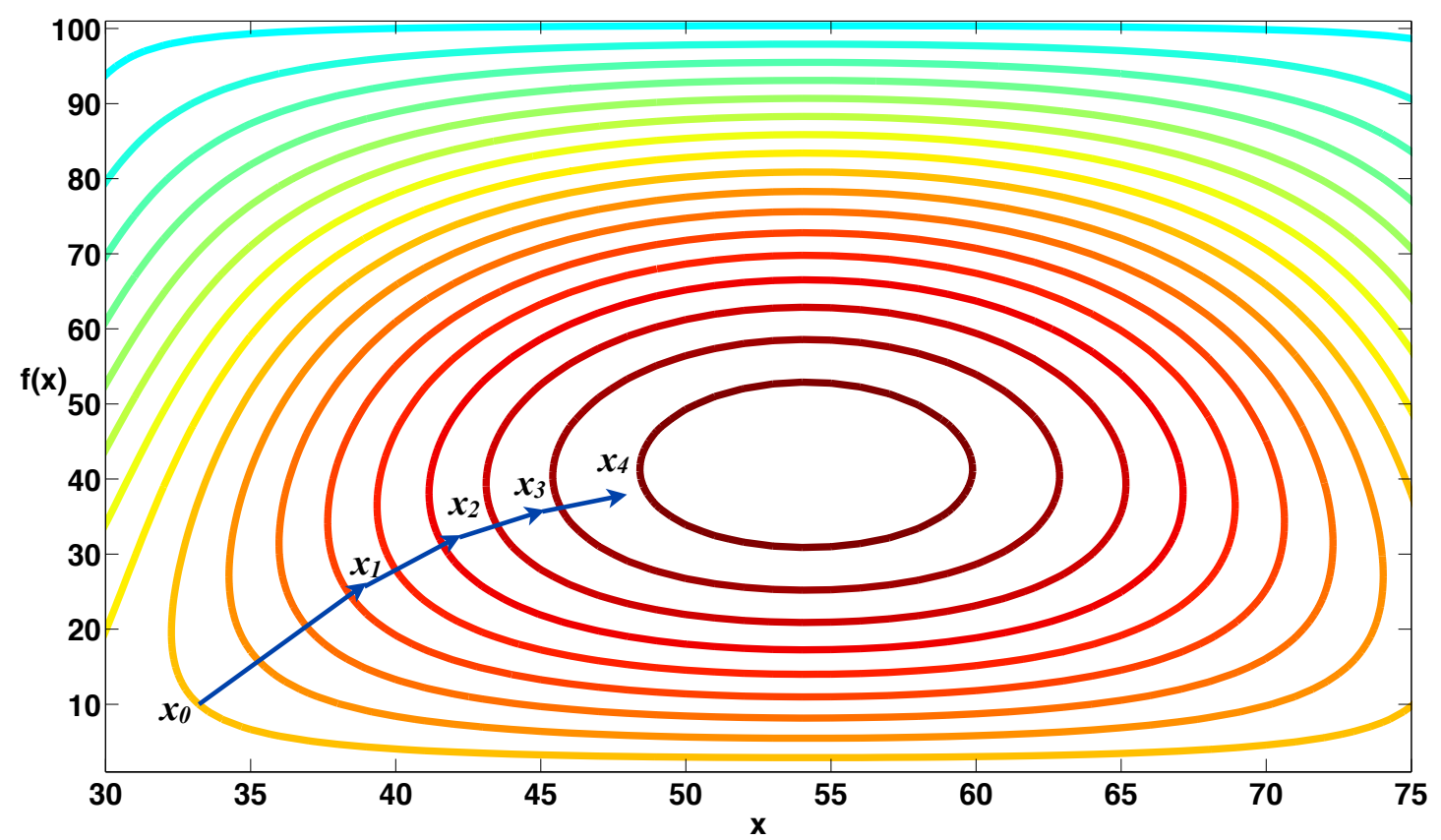

Figure 2.5: Illustration of steepest descent. The basis for the method is based on the observation that a continuous function should decrease in the direction proportional to the negative of the gradient.

\subsubsection{Implementation of the MHZ model: least-squares}

Typically applied as an approach to solving an overdetermined system of equations, the least squares method, minimises the sum of the squares of the residuals. Essentially, the least squares method reduces the errors between the data and the best approximating function (Burden and Douglas, 2005; Aster et al., 2005). For $n$ data pairs $\left(x_{1}, y_{1}\right),\left(x_{2}, y_{2}\right), \ldots,\left(x_{n}, y_{n}\right)$, if $\mathbf{a}=\left[\begin{array}{llll}a_{0} & a_{1} & \cdots & a_{m}\end{array}\right]^{T}$ is a solution to the normal equations

$$
\left(M^{T} M\right) \mathbf{a}=M^{T} Y
$$


where

$$
Y=\left[\begin{array}{c}
y_{1} \\
y_{2} \\
\vdots \\
y_{n}
\end{array}\right] \& M=\left[\begin{array}{cccc}
f_{0}\left(x_{1}\right) & f_{1}\left(x_{1}\right) & \ldots & f_{m}\left(x_{1}\right) \\
f_{0}\left(x_{2}\right) & f_{1}\left(x_{2}\right) & \cdots & f_{m}\left(x_{2}\right) \\
\vdots & \vdots & & \vdots \\
f_{0}\left(x_{n}\right) & f_{1}\left(x_{n}\right) & \cdots & f_{m}\left(x_{n}\right)
\end{array}\right]
$$

then the best approximating function (fit) to the data is given by (Nicholson, 1990)

$$
f(x)=a_{0} f_{0}(x)+a_{1} f_{1}(x)+\ldots+a_{m} f_{m}(x) .
$$

For an invertible matrix $M$, the solution to a is given by (Nicholson, 1990)

$$
\mathbf{a}=\left(M^{T} M\right)^{-1} M^{T} Y .
$$

Despite the fact that 2.25 trends towards non-linearity as $\sigma$ increases, one can apply linear least squares method by reframing 2.25 as a linear model

$$
y=a_{0}+a_{1} x
$$

where $y=\log _{2}\left|a_{i}\right|+j, a_{0}=\log _{2}|A|$ and $a_{1}=\frac{\alpha-1}{2}$. The solution to 2.36 is given by (Burden and Douglas, 2005)

$$
a_{0}=\frac{\sum_{i=1}^{n} x_{i}^{2} \sum_{i=1}^{n} y_{i}-\sum_{i=1}^{n} x_{i} y_{i} \sum_{i=1}^{n} x_{i}}{n\left(\sum_{i=1}^{n} x_{i}^{2}\right)-\left(\sum_{i=1}^{n} x_{i}\right)^{2}}
$$

and

$$
a_{1}=\frac{n \sum_{i=1}^{n} x_{i} y_{i}-\sum_{i=1}^{n} x_{i} \sum_{i=1}^{n} y_{i}}{n\left(\sum_{i=1}^{n} x_{i}^{2}\right)-\left(\sum_{i=1}^{n} x_{i}\right)^{2}}
$$

where $n$ is the number of data points. 


\section{Chapter 3}

\section{Algorithms for estimating regularity and smoothness}

An accurate estimation of the Lipschitz regularity of a seismic trace is regarded as a highly desirable goal. The linear signal model based on the continuous wavelet transform can be used for estimating the Lipschitz exponent associated with delta type or step like functions. For such events, we can estimate the Lipschitz exponent by measuring the slope or forming the objective function associated with the linear signal model.

However, for closely spaced signal events or seismic events that experience smoothness due to absorption, the linear model is impractical, thus the need for a non-linear signal model. Nevertheless, the linear signal model is applied to verify the properties of the Lipschitz exponent and used as a framework for the more practical non-linear signal model.

\subsection{The Lipschitz regularity of a spike}

For single, isolated events with an associated Lipschitz value of $\alpha=-1,0$ or 1 , the linear model given by

$$
\log _{2}\left|\mathbf{W}_{s} f(x)\right| \leq \log _{2}|A|+\alpha j
$$

reduces the computation to a simple slope estimation. To verify the accuracy of the model, one needs to compute the continuous wavelet transform and modulus maxima of a function with a known Lipschitz value. The corresponding theoretical Lipschitz value of the delta function is equal to $\alpha=-1$. Figures 3.1, 3.2 (a) and (b) represent the input function, the continuous wavelet transform and corresponding modulus maxima respectively. 


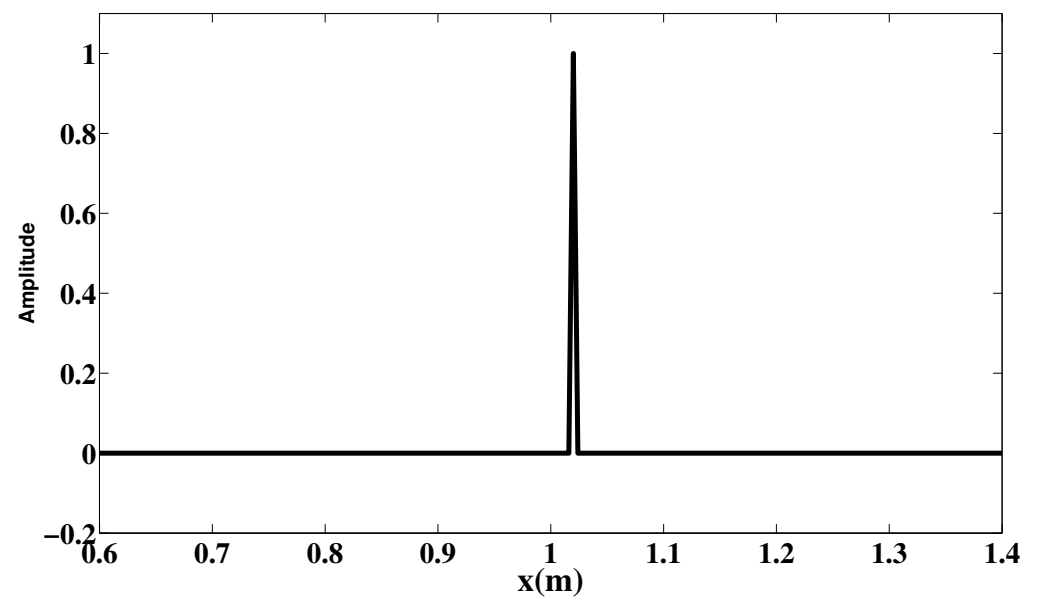

Figure 3.1: Original input signal 


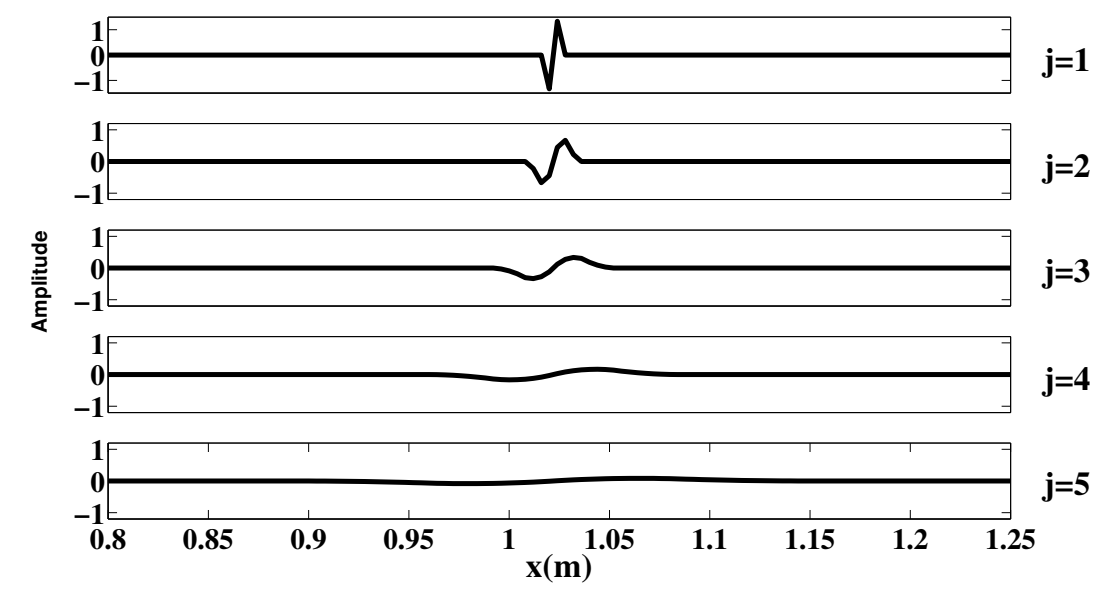

(a)

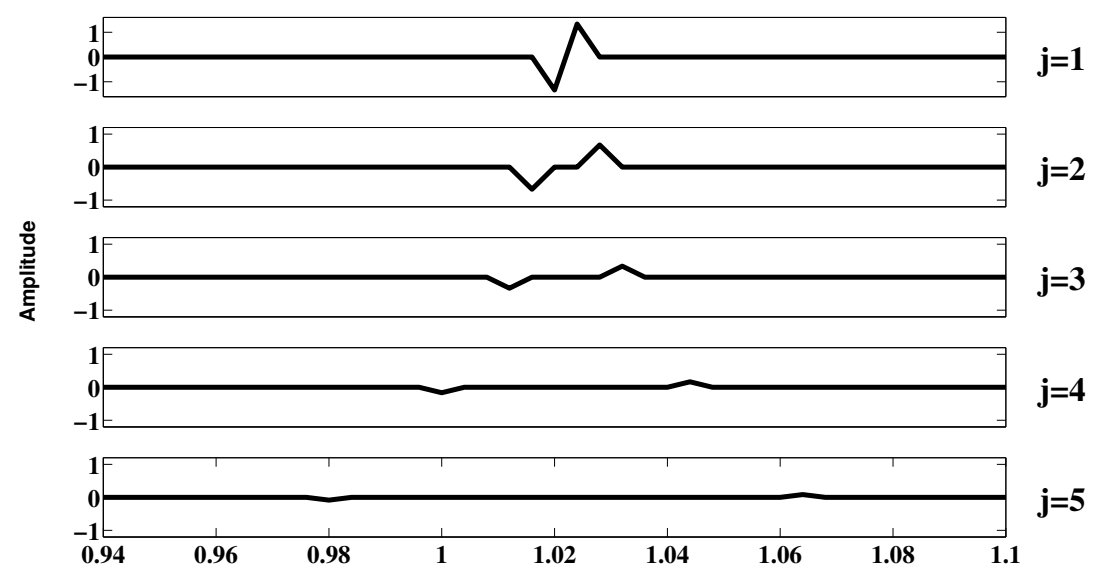

(b)

Figure 3.2: (a) Corresponding continuous wavelet transform at each scale $s=2^{j}$ (b) Corresponding modulus maxima of original signal at each scale $s=2^{j}$

Based on the theoretical properties of the delta function, an inverse correlation exists between modulus maxima values and the continuous wavelet transform scales. Clearly, such behaviour is observed in Figure 3.3, where the scale index ranges from $j=1,2, . ., 5$. Plotting the logarithm of the modulus maxima against scale index and subsequently computing the slope yields, one obtains an estimate for the Lipschitz exponent, which is equal to -0.998 , a very close estimate compared to the theoretical value of $\alpha=-1$. Furthermore, the intercept 
provides an estimate for $A$, which is equal to 2.333 (one should add a unit value of 1 to the estimated value of $A$, since $x$-axis starts at 1 ). Additionally, forming the objective function (Figure 3.4) and minimising yields 2.44 and -1.0004 for $A$ and $\alpha$ respectively.

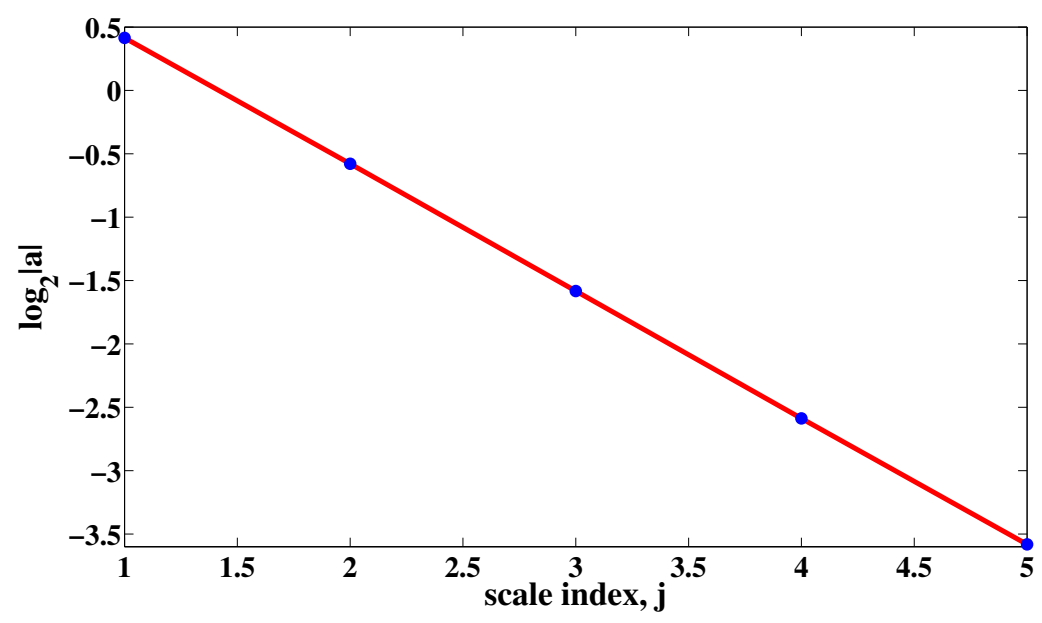

Figure 3.3: $\log _{2}|a|$ vs scale index, $j$

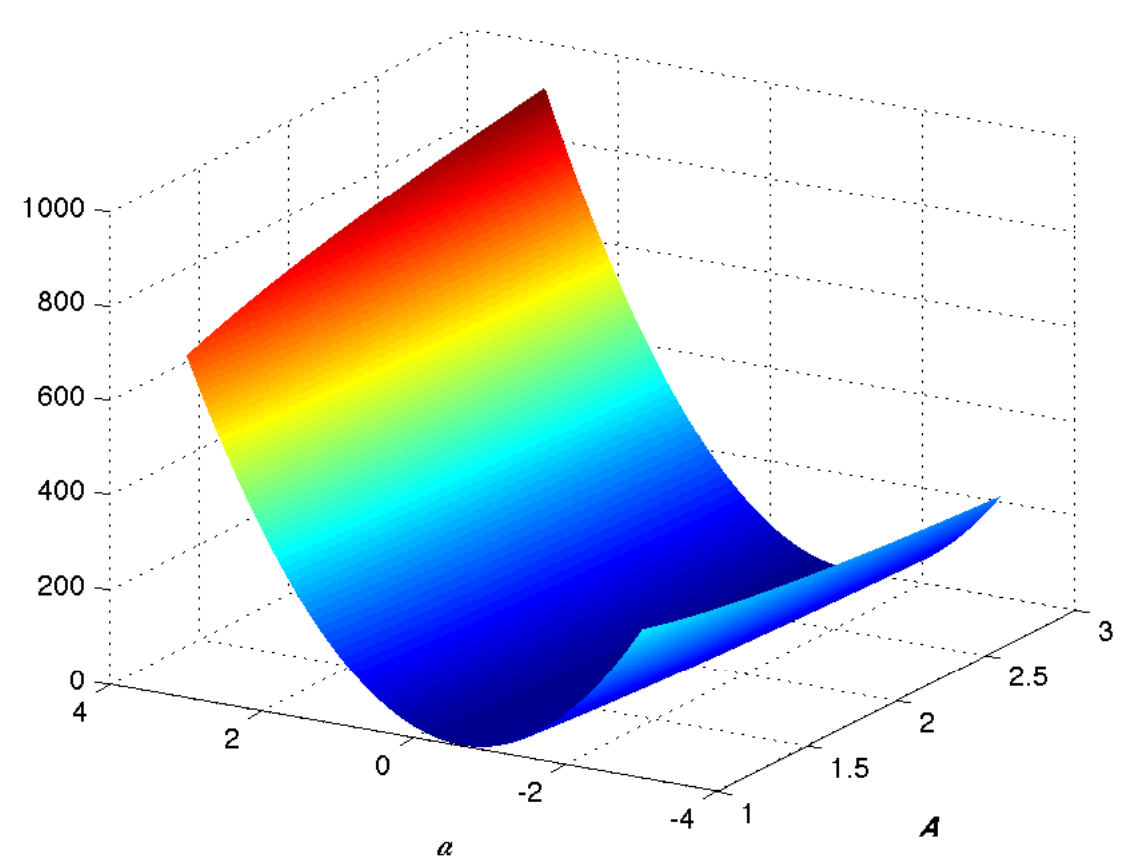

Figure 3.4: Two parameter objective function. The $x$ and $y$ axes represent the values for $A$ and $\alpha$ respectively 


\begin{tabular}{|cccccc|}
\hline$j$ & 1 & 2 & 3 & 4 & 5 \\
\hline$\left|a_{i}\right|$ & 1.3333 & 0.6696 & 0.3337 & 0.1667 & 0.0835 \\
\hline $\log _{2}\left|a_{i}\right|$ & 0.4150 & -0.5785 & -1.5832 & -2.5881 & -3.5821 \\
\hline
\end{tabular}

Table 3.1: Corresponding maxima modulus values at each scale, $s=j$ for the delta function

\subsection{The Lipschitz regularity of a step and ramp function}

Theoretically, integration increases the associated Lipschitz value of a particular function by

1. By integrating the delta function, one obtains the unit step function or the Heaviside function defined by the following

$$
H(x)= \begin{cases}0 & x<0 \\ \frac{1}{2} & x=0 . \\ 1 & x>0\end{cases}
$$

Furthermore, integrating the Heaviside function results in the ramp function, defined by the following

$$
R(x)= \begin{cases}x & x \geq 0 \\ 0 & x<0\end{cases}
$$

hence, based on the theoretical properties associated with the Lipschitz exponent one would expect an associated Lipschitz value of 0 and 1 for the Heaviside and the ramp function respectively.

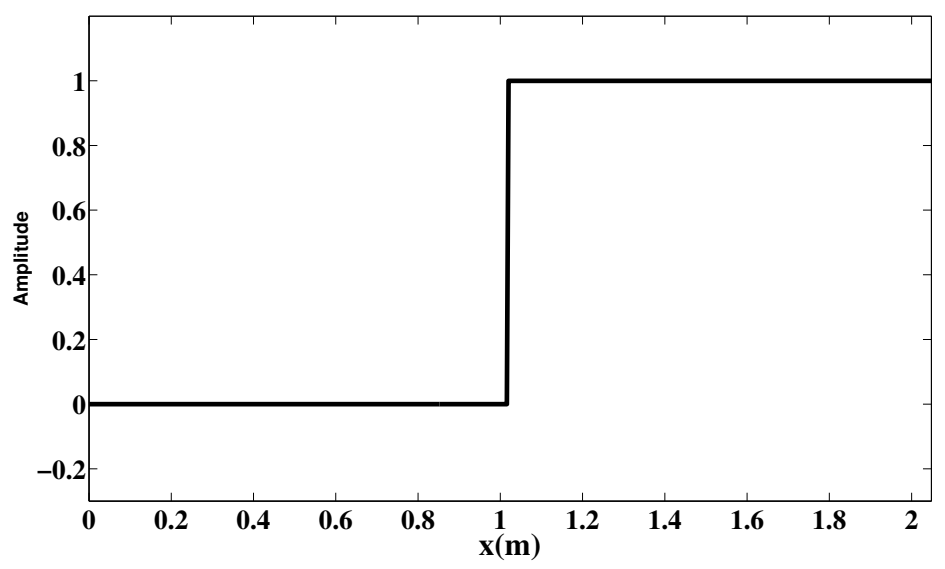




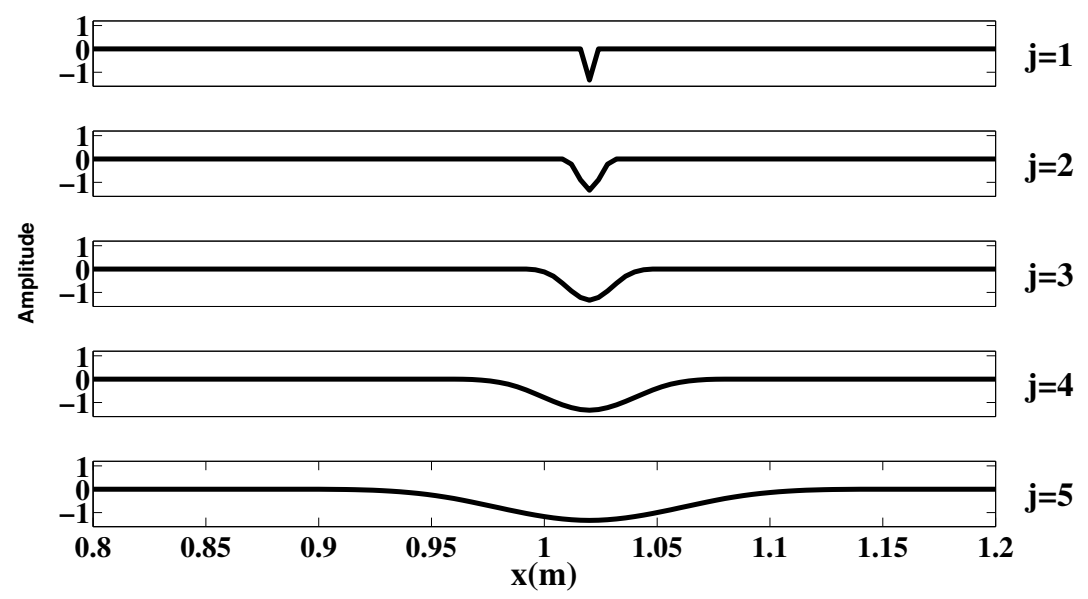

(a)

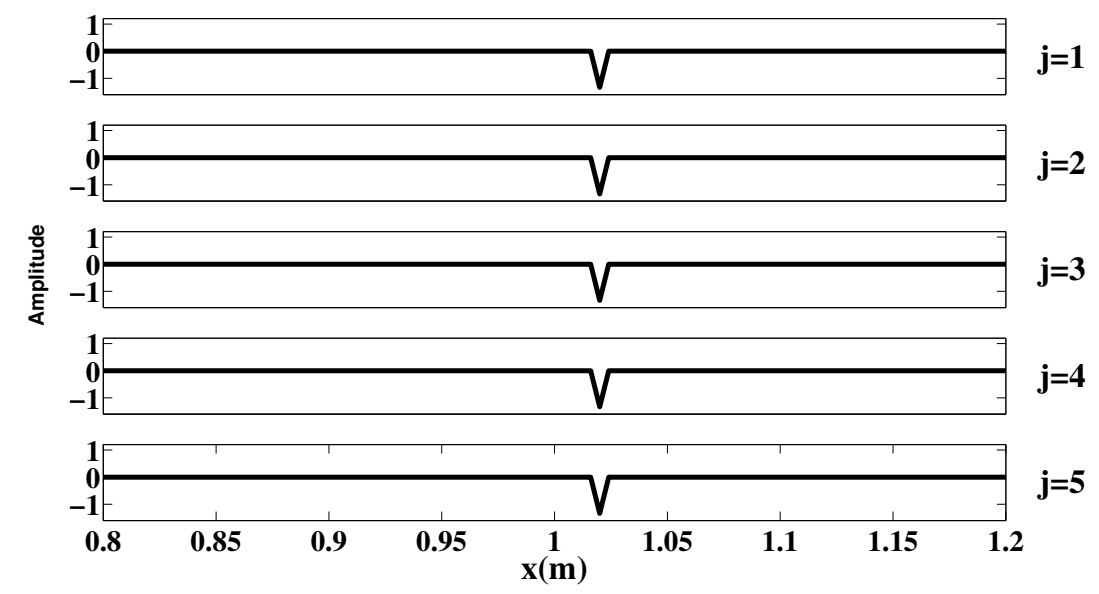

(b)

Figure 3.5: (a) Continuous wavelet transform at each scale $s=2^{j}$ for Heaviside function (b) Corresponding modulus maxima values at each scale $s=2^{j}$

As expected, one obtains an almost constant maxima modulus values for the Heaviside function, resulting in a constant slope and increasing maxima modulus values for the ramp function, yielding a positive slope. Similar to the case study for the delta function, plotting the maxima modulus values against scale and computing the slope yields a Lipschitz value of $\alpha=-6.50 \mathbf{e}-04$ and $\alpha=1.462$ for the Heaviside and ramp function respectively. However, estimating the Lipschitz exponent from the slope requires a degree of caution. In contrast 
to the delta function, it is clearly evident from Figures 3.6 and 3.7 that the slopes contain a degree of error. For the Heaviside function, the Lipschitz values $\alpha$ vary from -0.0010 to -0.0044 . To reduce the errors, one needs to form the objective function

$$
\phi(\alpha, A)=\sum_{i, j=1}^{n}\left[\log _{2}\left|a_{i}\right|-\left(\log _{2}|A|+\log _{2}\left(s_{j}\right)\right)\right]^{2},
$$

and subsequently minimise to find the corresponding $A$ and $\alpha$ values. Based on this method, one obtains $\alpha=0.00$ and $\alpha=0.9258$. Additionally minimisation yields the following values of $A=1.1138$ and $A=6.3440$. The value of $A$ apparently depends on the input function and may be the measure of the energy at the lowest scale (as the value differs for the delta function, Heaviside and ramp function). However without further study we can not state with certainty if $A$ is dependent on the input function.

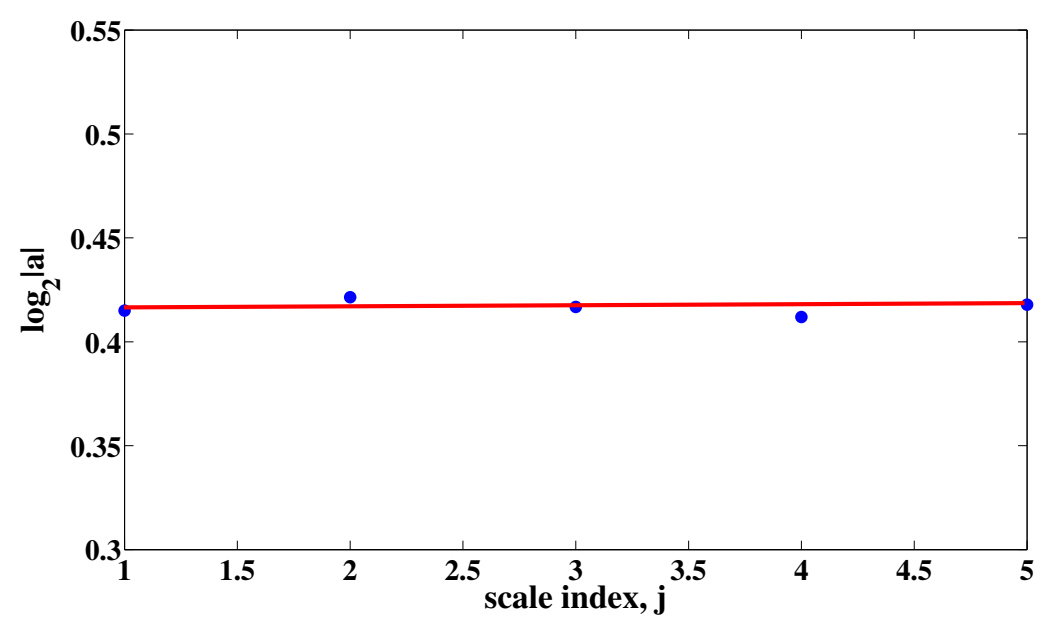

Figure 3.6: $\log _{2}|a|$ vs scale index, $j$ for the Heaviside function 


\begin{tabular}{|cccccc|}
\hline$j$ & 1 & 2 & 3 & 4 & 5 \\
\hline$\left|a_{i}\right|$ & 1.3333 & 1.3393 & 1.3350 & 1.3304 & 1.3359 \\
\hline $\log _{2}\left|a_{i}\right|$ & 0.4150 & 0.4215 & 0.4168 & 0.4119 & 0.4179 \\
\hline
\end{tabular}

Table 3.2: Modulus maxima values at each scale index, $j$ for the Heaviside function

\begin{tabular}{|cccccc|}
\hline$j$ & 1 & 2 & 3 & 4 & 5 \\
\hline$\left|a_{i}\right|$ & 0.0133 & 0.0357 & 0.0777 & 0.1584 & 0.3200 \\
\hline $\log _{2}\left|a_{i}\right|$ & -6.2288 & -4.8074 & -3.6865 & -2.6582 & -1.6439 \\
\hline
\end{tabular}

Table 3.3: Modulus maxima values at each scale, $j$ for the ramp function

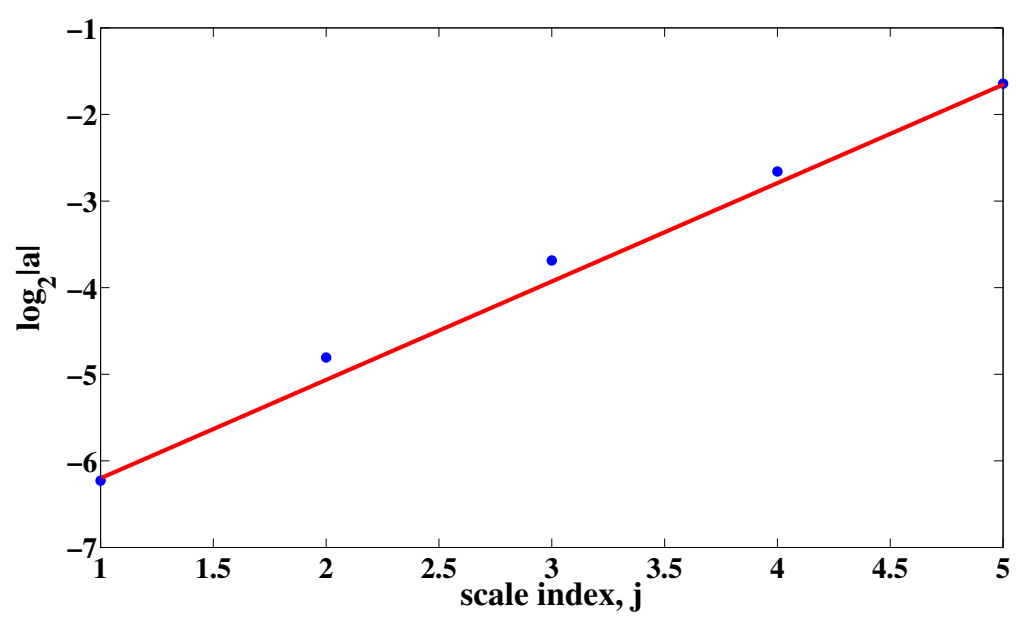

Figure 3.7: $\log _{2}|a|$ vs scale index, $j$ for the ramp function

\subsection{The effect of closely-spaced events}

For a medium with a relatively low $Q$ value, a seismic pulse gradually obtains spectral characteristics of a Gaussian, hence, plotting the modulus maxima value against the scale provides a degree of insight into to the extent of absorption. Figure 3.8 represents the "evolution" of $\log _{2}|a|$ vs scale index $j$, for a pulse travelling within an absorptive medium. Clearly, the modulus maxima values trend towards non-linearity with increasing $\sigma$. 


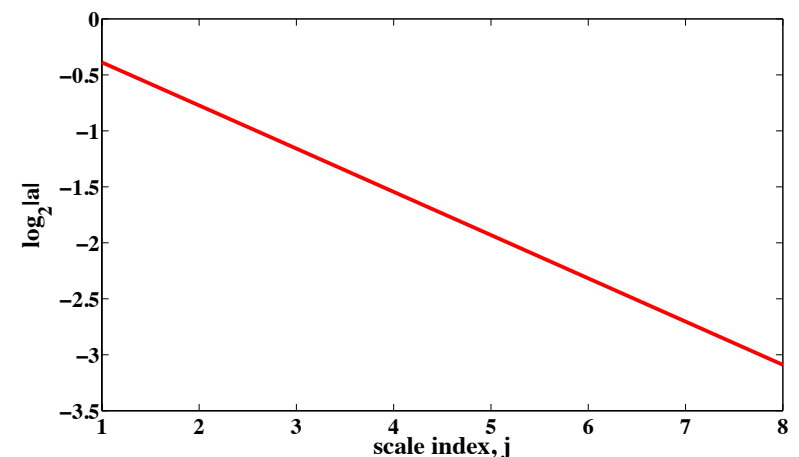

(a)

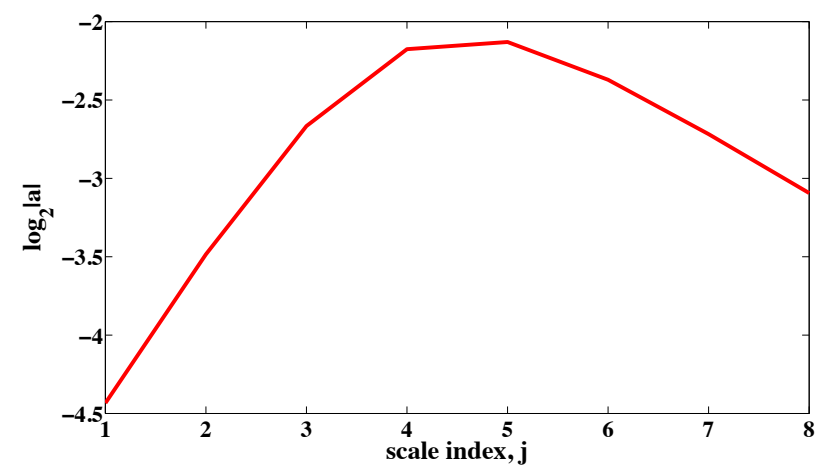

(c)

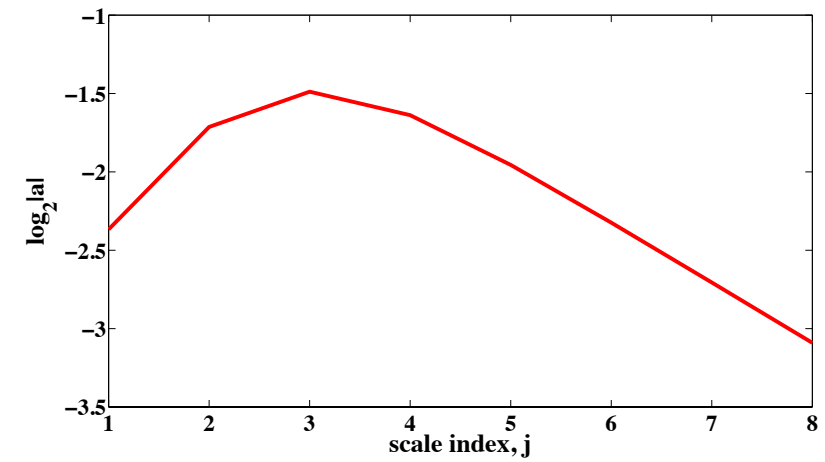

(b)

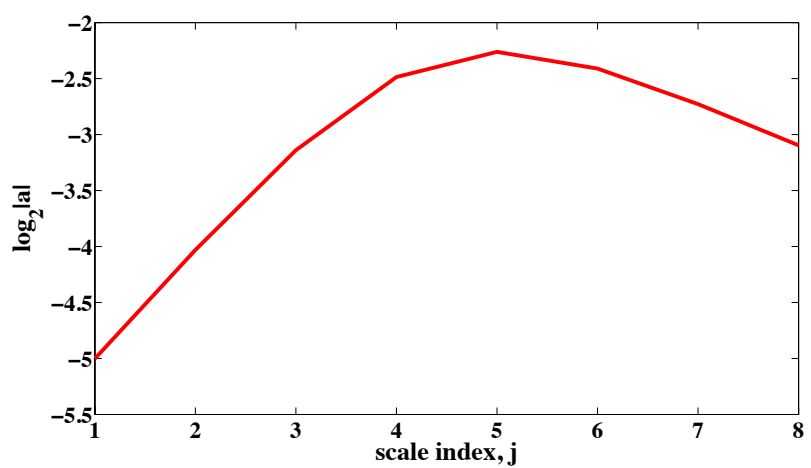

(d)

Figure 3.8: $\log _{2}|a|$ vs scale index, $j$ for a pulse with increasing $\sigma$ values from (a) to (d)

Despite the benefits, several limitations associated with the modulus maximum model, such as closely spaced events, restrict and complicate our ability to accurately estimate and analyse the corresponding Lipschitz regularity. For two closely placed events, the modulus maxima values start to merge and represent a single event with increasing scale (illustrated in Figure 3.10), hence rendering any form of distinction between two events almost impossible. One possible solution is to impose some sort of thresholding on the scale. However such a procedure not only becomes complicated due to the behaviour of a Gaussian function, but also leads to criticism of visual bias. Unfortunately, as illustrated in Figure 3.11, one can not observe a dominant trend or pattern in order to impose a threshold on the scale. 
For two Gaussians with $\sigma=0.01$ and $\sigma=0.05$, it is unclear how one could restrict the scale without the corresponding modulus values merging (at a certain scale) in order to differentiate between the two events.

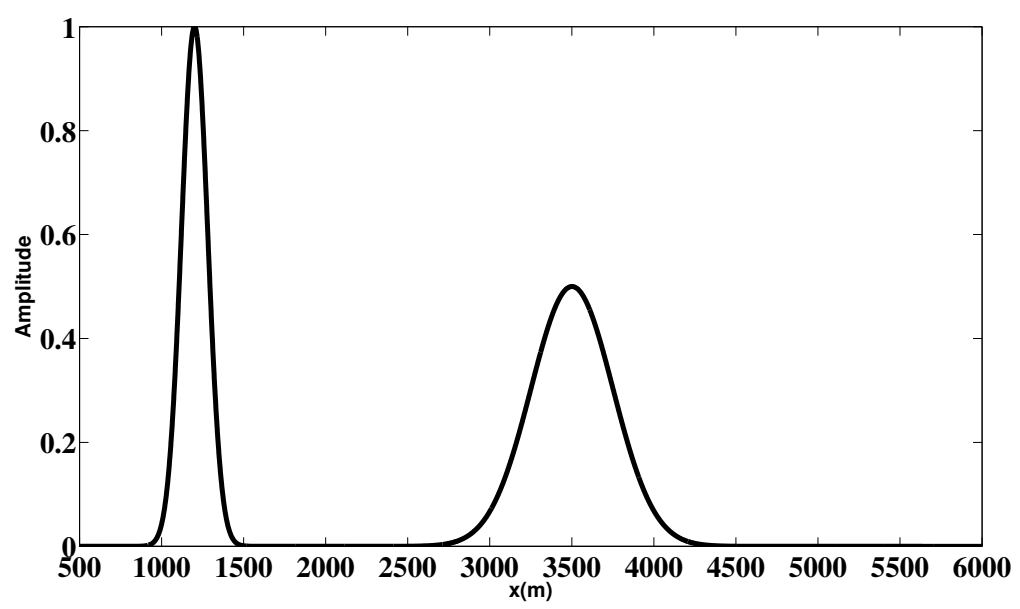

Figure 3.9: Two closely spaced Gaussians representing the input signal

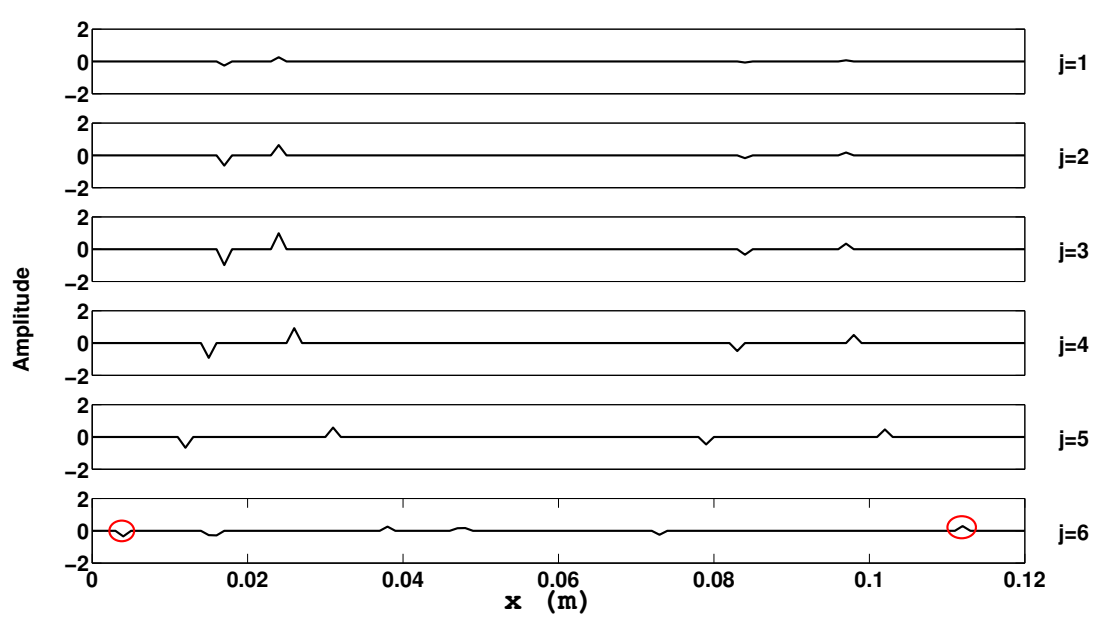

Figure 3.10: Corresponding modulus maxima values. From $j=1, . ., 5$ we have two max values corresponding to two distinct events. However at $j=6$ the two max values merge, thus representing a single event 


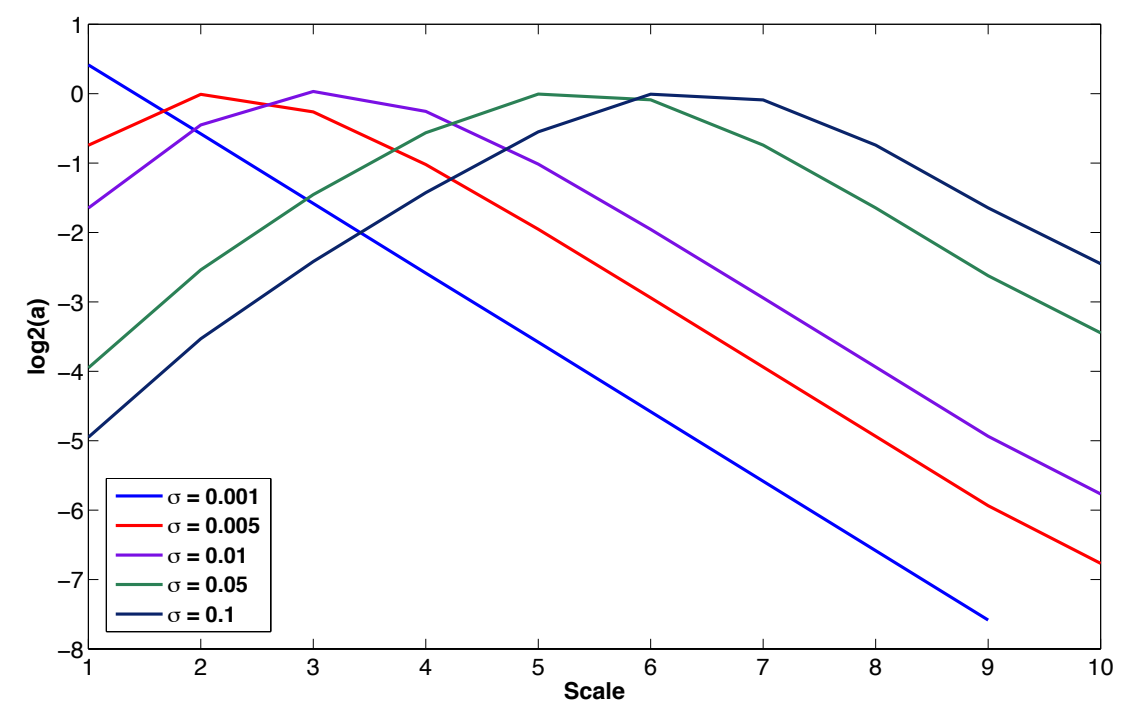

Figure 3.11: $\log _{2}|a|$ vs scale, $s=j$ for increasing $\sigma$ values. The plot corresponding to a pulse with increasing smoothness ( $\sigma$ values) due to absorption. 


\section{Chapter 4}

\section{Determining Q from the regularity of VSP data: synthetic study}

\subsection{Vertical seismic profile (VSP) data}

Over the last few decades, vertical seismic profiling (VSP) has become an indispensable tool in seismic surveys. By utilising surface sources and borehole receivers, a vertical seismic profile refers to the measurement(s) of a seismic signal generated at the surface and recorded at various vertical depth locations (Hardage, 1983; Hinds et al., 1996; Sergio and Ulrych, 1988). The procedure provides understanding of reflection and transmission processes within the earth and insight into the fundamental properties of seismic wave propagation, which in turn enhances and improves structural and lithological interpretations of seismic surface recordings (Hardage, 1983).

The applications of VSP data include the separation of up-doing and down-going wave components (due to small amplitudes of up-going waves), identification of multiples, measurement of compressional and shear wave velocities, location of fault planes and estimation of reflector $\operatorname{dip}(\mathrm{s})$ among others (Sergio and Ulrych, 1988; Hardage, 1983). Based on the geometrical configuration, a VSP survey is categorised into near-offset or zero-offset and far-offset. Our primary focus is on zero-offset VSP, which refers to the vertical alignment of source and receiver locations which is illustrated in Figure 4.1 (Hinds et al., 1996). It should be noted that for visual clarity, the source-receiver offset has been exaggerated.

In order to assess the effects of absorption on a given pulse and establish an empirical relation between the Lipschitz exponent $\alpha$ and the loss factor $Q$, a synthetic zero-offset VSP model for a single layer with varying velocity, depth and $Q$ values is constructed. Applying 
the continuous wavelet transform to the given impulse response and subsequently estimating the corresponding modulus maxima values permits us to use the methods developed in the previous two chapters to estimate the corresponding Lipschitz values. The evolution of the Lipschitz values with depth is then to provide the necessary mathematical mapping relation between $Q$ and $\alpha$.

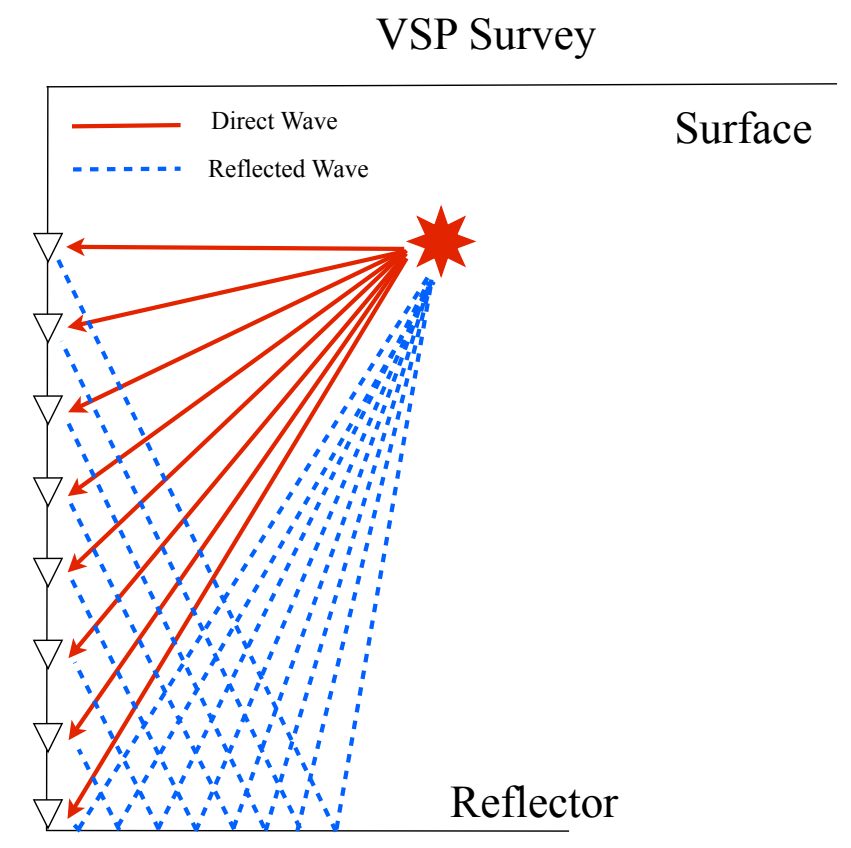

Figure 4.1: Zero-offset VSP field layout.

\subsection{The regularity and smoothness of VSP events}

For the initial VSP model, the wave velocity is set to a fixed value of $v=2500 \mathrm{~m} / \mathrm{s}$, whereas the receiver depth and $Q$ vary from $300 m$ to $1600 m$ and 10 to 200 respectively. The wavelet scale index ranges from $j=1$ to $j=6$ with the sample rate and sample number set to $4 m s$ and 512 respectively. As one would expect with decreasing $Q$, the pulse starts to lose amplitude and broaden in width (illustrated in Figure 4.2). Hence, the expected Lipschitz value should shift from -1 to values closer to 0 . In order to estimate the Lipschitz values, we need to minimise the following multivariable objective function (detailed description of 
the following relation provided in section 2.4.1)

$$
\phi(A, s, \sigma)=\sum_{i, j=1}^{n}\left[\log _{2}\left|a_{i}\right|-\log _{2}|A|-j-\frac{\alpha-1}{2} \log _{2}\left(\sigma^{2}+2^{2 j}\right)\right]^{2} .
$$

\subsubsection{Steepest descent estimation}

For a receiver located at depth $z=270 \mathrm{~m}$ and the loss factor set to $Q=20$, the steepest descent method converges to -0.4501 and 0.9893 for $\alpha$ and $A$ respectively. Based on the extent of absorption, the estimated value for the Lipschitz exponent $\alpha$ is clearly within an acceptable range, close to 0 . However, based on the results given in Table 4.1 it is clearly evident that the steepest descent method fails to provide an estimate for the variance $\sigma$. This could possibly be related to the topography of the objective function, such that a global minimum may not exist along the $\sigma$-axis. Nevertheless, it is difficult to provide a plausible explanation without further study. Alternatively, reducing absorption by setting the loss factor to $Q=50$ and $Q=100$, should decrease the value of the Lipschitz exponent $\alpha$. The steepest descent converges to $\alpha=-0.7089$ and $\alpha=-0.9858$ for $Q=50$ and $Q=100$ respectively.

\begin{tabular}{|c|c|c|c|c|c|c|}
\hline & Guess 1 & Estimated & Guess 3 & Estimated & Guess 3 & Estimated \\
\hline$\alpha$ & -0.4 & -0.6806 & -0.2 & -0.4294 & -0.4 & -0.4501 \\
$A$ & 2 & 1.9506 & 1 & 0.9251 & 1 & 0.9838 \\
$\sigma$ & 1 & 1.0000 & 0.1 & 0.1000 & 0.01 & 0.0101 \\
$\nabla g$ & & 1.8306 & & 1.3772 & & 1.3134 \\
\hline
\end{tabular}

Table 4.1: Estimated values using the steepest descent for a receiver located at $z=270 \mathrm{~m}$ and $Q=20$.

Although the steepest descent method provides an acceptable value for the Lipschitz exponent, one should not ignore the limitations and drawbacks associated with this method. First and foremost, the steepest descent method tends to be a time consuming procedure with the gradient $\nabla g$ serving as the only guide in order to improve the initial guess values and subsequent convergence. Furthermore as the extent of absorption increases, the method provides an estimate for the Lipschitz exponent that may seem suspicious. Setting the 


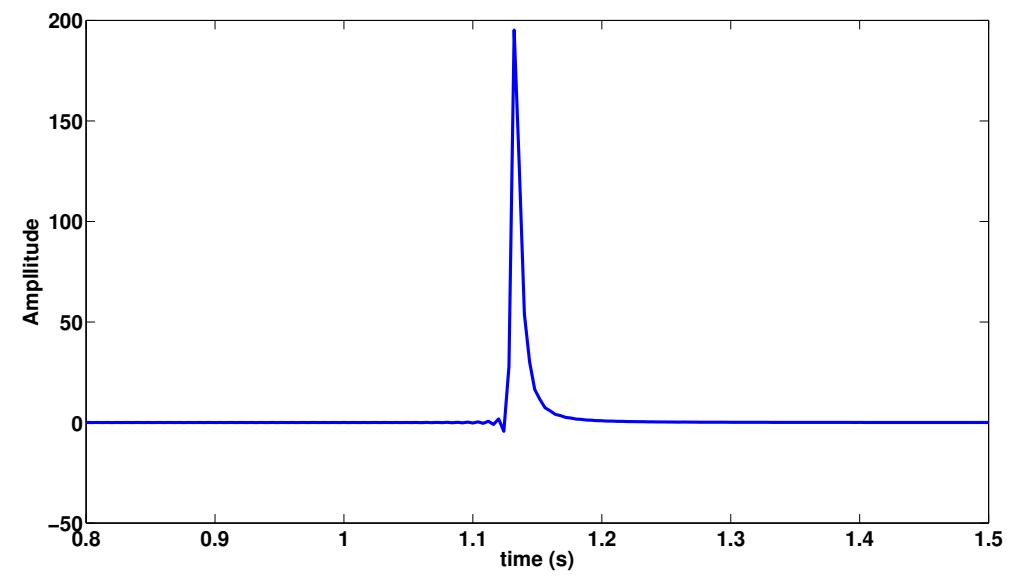

(a)

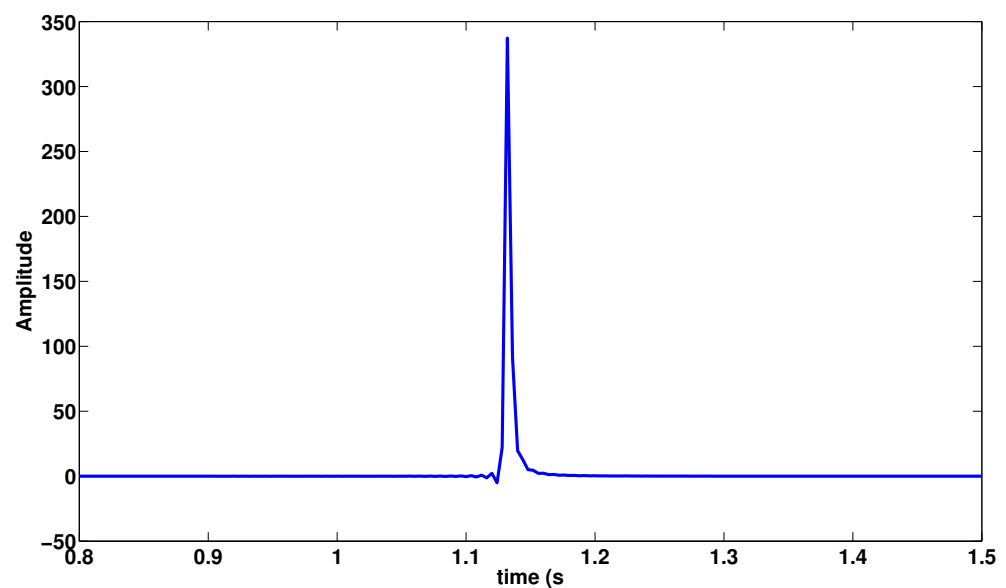

(b)

Figure 4.2: Direct arrivals corresponding to a receiver at $z=270 m$ with (a) $Q=20$ (b) $Q=50$.

velocity to $v=1500 \mathrm{~m} / \mathrm{s}, Q=20$ and receiver depth to $z=450 \mathrm{~m}$ the steepest descent converges to a value of $\alpha=-0.5460$. Based on the theoretical model and previous results, one would expect a Lipschitz value closer to $\alpha \simeq 0$. It should also be noted that the steepest descent fails to provide any information related to the third parameter .

\subsubsection{Scales to be used and avoided}

As an alternative to the steepest descent, one may consider linearising the problem by analysing the dominant trend or behaviour of the modulus maxima values against the wavelet 


\begin{tabular}{|c|c|c|c|c|}
\hline$Q=50$ & $\alpha$ & $A$ & $\sigma$ & $\nabla g$ \\
\hline Optimal Guess & -0.65 & 1 & 0.001 & \\
Estimated Value & -0.70 & 1.4876 & 0.001 & 0.4358 \\
\hline \multicolumn{5}{|c|}{$(\mathrm{a})$} \\
\hline$Q=100$ & $\alpha$ & $A$ & $\sigma$ & $\nabla g$ \\
\hline Optimal Guess & -0.90 & 3 & 0.001 & \\
Estimated Value & -0.9858 & 2.9675 & 0.001 & 0.1556 \\
\hline
\end{tabular}

(b)

Table 4.2: Estimated values using steepest descent for receiver located at $z=270 \mathrm{~m}$ and (a) $Q=50$, (b) $Q=100$.

\begin{tabular}{|c|c|}
\hline & Estimated Value \\
\hline$\alpha$ & -0.5460 \\
$A$ & 0.3626 \\
$\sigma$ & Not determined \\
$\nabla g$ & 5.1412 \\
\hline
\end{tabular}

Table 4.3: Estimated values using steepest descent for receiver located at $z=450 \mathrm{~m}$ and $Q=20$.

scale. Setting velocity to $v=2500 \mathrm{~m} / \mathrm{s}$, loss factor to $Q=100$ and receiver depth to $z=270 \mathrm{~m}$ and subsequently applying the continuous wavelet transform on the obtained trace provides an estimate for the corresponding modulus maxima values. As expected, by plotting the logarithm of modulus maxima values against the wavelet scale one obtains a linear model. However (as illustrated in figure 4.3), it is clearly evident that the slope is slightly steeper from $j=2$ to $j=6$. Limiting the scale index to $j=(2-6)$ and subsequently computing the slope yields an estimate equal to $\alpha=-0.9550$ for the corresponding Lipschitz exponent. For the same model the estimated Lipschitz value obtained from the steepest descent method differs by -0.0308 .

However one should be aware and consider the limitations associated with this method. First and foremost, it is not possible to linearise the problem or observe a dominant trend or behaviour with increasing absorption. Clearly, as evident in Figure 4.4, with increasing absorption the obtained model $\left(\log _{2}\left|a_{i}\right|\right.$ vs scale index, $\left.j\right)$ trends towards non-linearity, hence regardless of the scale cut-off it is impossible to calculate or estimate a dominant slope value. Furthermore, restricting the model to a simple slope estimation leads to loss of information 
regarding the two additional parameters, $A$ and $\sigma$.

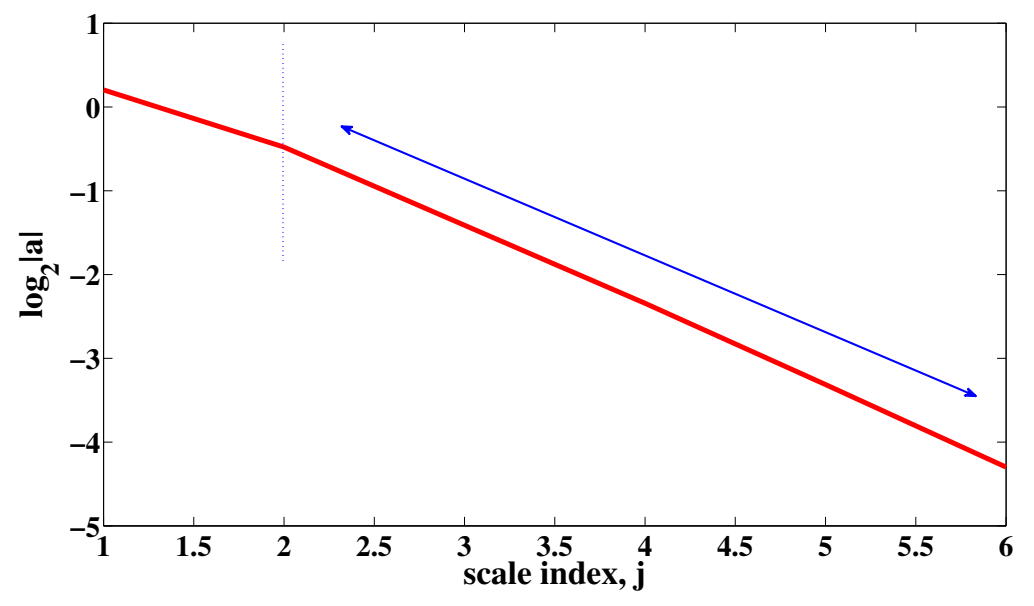

Figure 4.3: Logarithm of modulus maxima values vs scale index $j$. The dashed line and blue arrow indicate the scale index cut-off and number of scales used for measuring the slope respectively. 


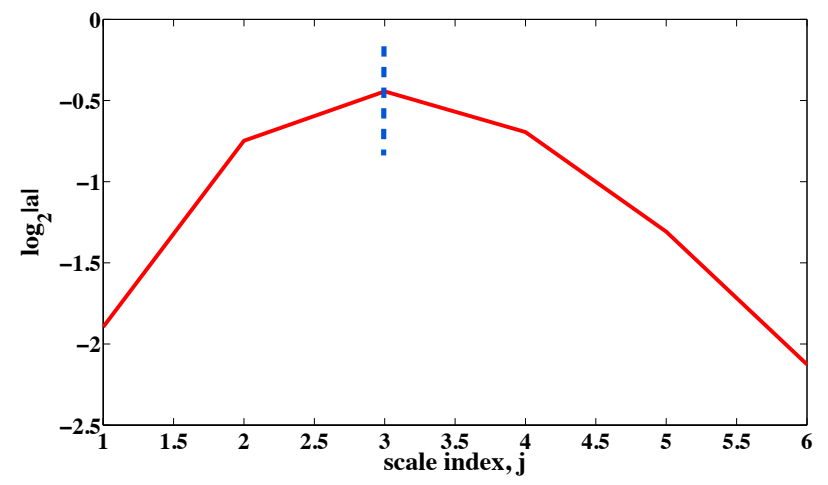

(a)

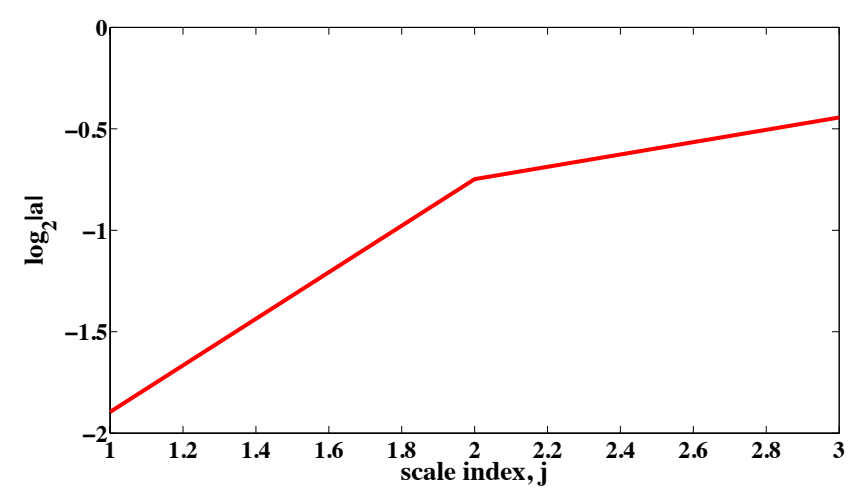

(b)

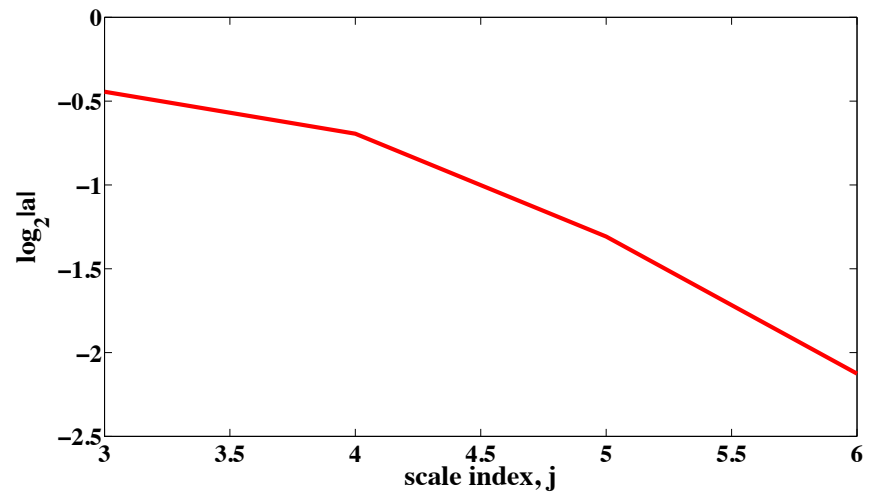

(c)

Figure 4.4: Logarithm modulus maxima values vs scale index $j$ for $z=710 \mathrm{~m}$ and $Q=20$. The dashed line indicates the scale index cut-off and the difficulty obtaining a dominant trend as illustrated in (b) and (c).

\subsection{A re-arrangement of the model}

In order to overcome the deficiencies, limitations and setbacks associated with the steepest descent or thresholding technique, one could re-arrange and pose the three parameter nonlinear model (mapping modulus maxima values to the Lipschitz exponent) given by

$$
\log _{2}\left|a_{j}\right|=\log _{2}|A|+j+\frac{\alpha-1}{2} \log _{2}\left(\sigma^{2}+2^{2 j}\right)
$$


as follows

$$
y=a_{0}+a_{1} x
$$

where $y=\log _{2}\left|a_{i}\right|-j, x=\log _{2}\left(\sigma^{2}+2^{2 j}\right), a_{0}=\log _{2}|A|$ and $a_{1}=-\frac{\alpha-1}{2}$. It should be noted that for our analysis, the indices $i$ and $j$ are equivalent, thus the index $i$ in $\log _{2}\left|a_{i}\right|$ has been replaced by $j$. Forming the objective function and minimising using the least squares method yields the following

$\log _{2}|A|=\frac{\sum_{j=1}^{n}\left[\log _{2}\left(\sigma^{2}+2^{2 j}\right)\right]^{2} \sum_{j=1}^{n}\left(\log \left|a_{j}\right|-j\right)-\sum_{j=1}^{n}\left[\log _{2}\left(\sigma^{2}+2^{2 j}\right)\right]\left(\log _{2}\left|a_{j}\right|-j\right) \sum_{j=1}\left[\log _{2}\left(\sigma^{2}+2^{2 j}\right)\right]}{n\left(\sum_{j=1}^{n}\left[\log _{2}\left(\sigma^{2}+2^{2 j}\right)\right]^{2}\right)-\left(\sum_{j=1}^{n}\left[\log _{2}\left(\sigma^{2}+2^{2 j}\right)\right]\right)^{2}}$

and

$$
\alpha=1-2 \frac{n \sum_{j=1}^{n}\left[\log _{2}\left(\sigma^{2}+2^{2 j}\right)\right]\left(\log _{2}\left|a_{j}\right|-j\right)-\sum_{j=1}^{n}\left[\log _{2}\left(\sigma^{2}+2^{2 j}\right)\right] \sum_{j=1}^{n}\left(\log _{2}\left|a_{j}\right|-j\right)}{n\left(\sum_{i=1}^{n}\left[\log _{2}\left(\sigma^{2}+2^{2 j}\right)\right]^{2}\right)-\left(\sum_{j=1}^{n}\left[\log _{2}\left(\sigma^{2}+2^{2 j}\right)\right]\right)^{2}},
$$

where $n$ is the number of data points. In essence, minimising equation 4.2 for a range of $\sigma$ values provides the best fit to the plot of $\log _{2}\left|a_{i}\right|$ vs $j$.

Figure 4.6 illustrates the maxima modulus values against wavelet scale corresponding to the direct arrivals at receiver locations $z=270 \mathrm{~m}, z=630 \mathrm{~m}, z=1230 \mathrm{~m}$ and $z=1470 \mathrm{~m}$ (Figure 4.5) where $Q=50$ and $v=2500 \mathrm{~m} / \mathrm{s}$. Taking into consideration the fact that non-linearity in Equation 4.2 stems from increasing $\sigma$ value, the least squares minimisation method not only captures the non-linear segment given in Figure 4.6, but provides an overall accurate fit to the data $\left(\log _{2}\left|a_{i}\right|\right.$ vs $\left.j\right)$. The total error between the data and least squares fit in Figure 4.6 (a) to (d) is $0.0034,0.0206,0.0177$ and 0.0438 respectively. It should be noted that the total error is calculated by the following expression

$$
E_{i}=\sum_{i=1}^{n}\left|y_{i}-p_{i}\right|^{2}
$$

where $y_{i}, p_{i}$ and $n$ represent the data points, estimated values and number of data points respectively. Furthermore, the least squares minimisation provides a relatively accurate 
estimate for all three parameters $\alpha, A$ and as indicated in Table 4.4. However one should be cautious of the first two $\alpha$ values in Table 4.4 corresponding to $Q=20$ and $Q=30$. Based on the theoretical property, the Lipschitz value $(\alpha)$ should decrease with increasing $Q$ (decreasing absorption), hence one should expect values closer to -1 compared to the successive values.

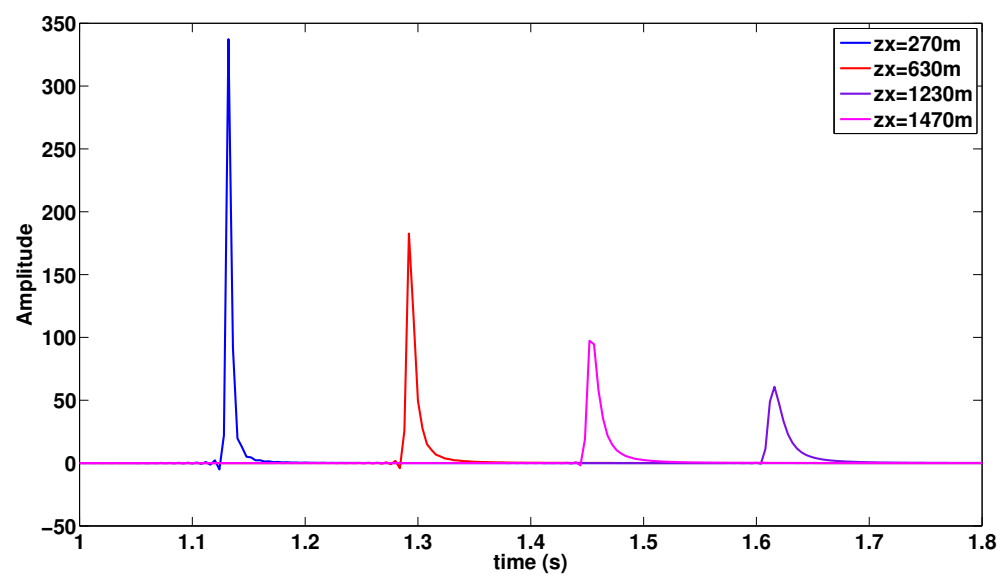

Figure 4.5: Direct arrivals corresponding to receivers located at $z=270 \mathrm{~m}, z=630 \mathrm{~m}, z=1230 \mathrm{~m}$ and $z=1470 m$ respectively with $Q=20$.

\subsection{The relationship between MHZ model parameters and $Q$}

Plotting $\alpha$ against $Q$, could provide greater insight into the possible relation between the Lipschitz exponent and absorption. As illustrated in Figure 4.7 (a) it is clearly evident that for the $\alpha$ and $Q$ values given in Table 4.4, a trivial function or mathematical description providing a mapping between $\alpha$ and $Q$ may not exist.

4.4.1 The relationship between the Lipschitz regularity $\alpha$ and $Q$

Based on the curve fitting methods and a certain degree of trial and error, in order to establish a mapping between $\alpha$ and $Q$ one might consider the following function

$$
\alpha=a_{1} Q^{m} \ln Q+a_{2} Q^{m}+a_{3} .
$$




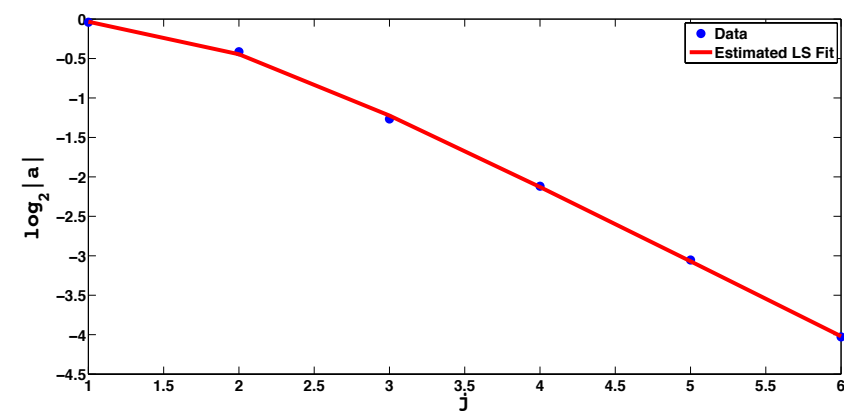

(a)

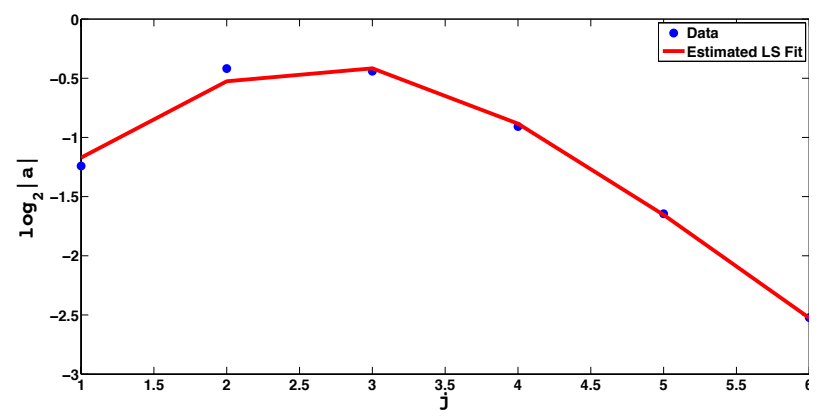

(c)

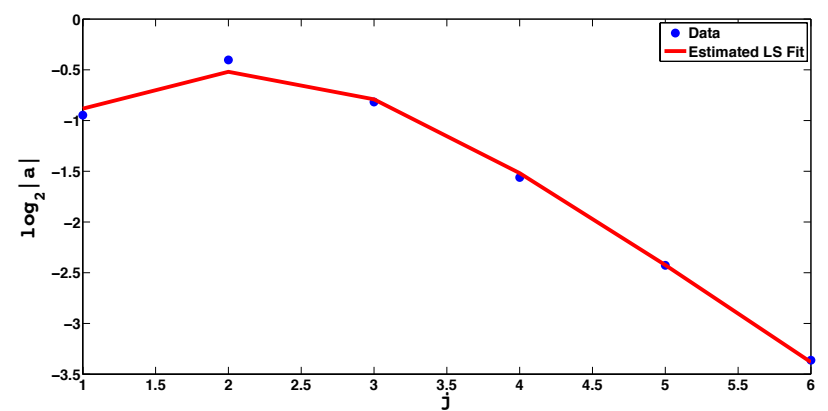

(b)

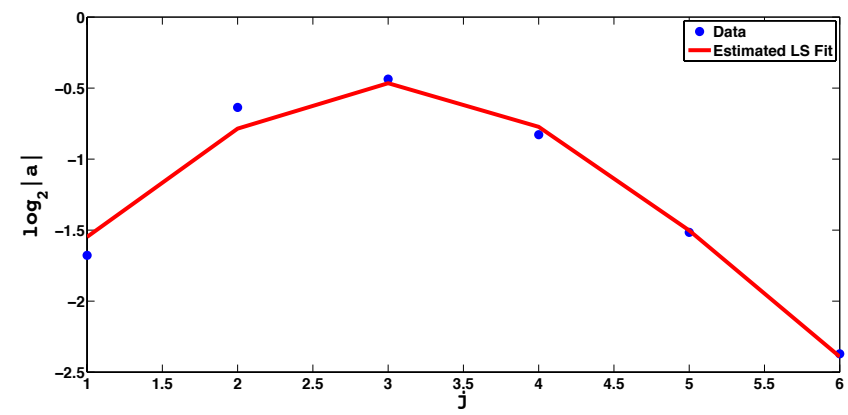

(d)

Figure 4.6: $\log _{2}\left|a_{i}\right|$ vs $j$ for direct arrival at (a) $z=270 \mathrm{~m}$ (b) $z=630 \mathrm{~m}$ (c) $z=1230 \mathrm{~m}$ (d) $z=1470 m$.

The solution to $a_{1}, a_{2}$ and $a_{3}$ is given by

$$
A=\left(M^{T} M\right)^{-1} M^{T} Y
$$

where

$$
A=\left[\begin{array}{c}
a_{1} \\
a_{2} \\
a_{3}
\end{array}\right], Y=\left[\begin{array}{c}
\alpha_{1} \\
\alpha_{2} \\
\vdots \\
\alpha_{n}
\end{array}\right]
$$




\begin{tabular}{|c|c|c|c|c|}
\hline$z=270 m$ & $\alpha$ & $A$ & $\sigma$ & TotalError, $E_{i}$ \\
\hline$Q=20$ & -0.9707 & 5.7788 & 4.3700 & 0.0177 \\
$Q=30$ & -0.9481 & 4.0218 & 2.7300 & 0.0040 \\
$Q=40$ & -0.9472 & 3.4721 & 2.0600 & 0.0032 \\
$Q=50$ & -0.9531 & 3.2482 & 1.7200 & 0.0034 \\
$Q=60$ & -0.9610 & 3.1515 & 1.5200 & 0.0022 \\
$Q=70$ & -0.9659 & 3.0753 & 1.2600 & 0.0015 \\
$Q=80$ & -0.9702 & 3.0241 & 1.2600 & 0.0011 \\
$Q=90$ & -0.9743 & 2.9924 & 1.1800 & 0.0009 \\
$Q=100$ & -0.9771 & 2.9618 & 1.1100 & 0.0007 \\
$Q=110$ & -0.9792 & 2.9354 & 1.0500 & 0.0006 \\
$Q=120$ & -0.9811 & 2.9146 & 1.0000 & 0.0005 \\
$Q=130$ & -0.9820 & 2.8907 & 0.9500 & 0.0004 \\
$Q=140$ & -0.9841 & 2.8830 & 0.9200 & 0.0004 \\
$Q=150$ & -0.9848 & 2.8643 & 0.8800 & 0.0003 \\
$Q=160$ & -0.9858 & 2.8531 & 0.8500 & 0.0003 \\
$Q=170$ & -0.9865 & 2.8407 & 0.8200 & 0.0003 \\
$Q=180$ & -0.9877 & 2.8355 & 0.8000 & 0.0002 \\
$Q=190$ & -0.9886 & 2.8294 & 0.7800 & 0.0002 \\
$Q=200$ & -0.9893 & 2.8226 & 0.7600 & 0.0002 \\
\hline
\end{tabular}

Table 4.4: Corresponding estimated values for direct arrival at $z=270 \mathrm{~m}$ with $Q=50$ and $v=2500 \mathrm{~m} / \mathrm{s}$.

and

$$
M=\left[\begin{array}{ccc}
Q_{1}^{m} \ln Q_{1} & Q_{1}^{m} & 1 \\
Q_{2}^{m} \ln Q_{2} & Q_{2}^{m} & 1 \\
\vdots & \vdots & \vdots \\
Q_{n}^{m} \ln Q_{n} & Q_{n}^{m} & 1
\end{array}\right]
$$

Hence, based on Equation 4.7 and the $Q$ and $\alpha$ given in Table 4.4, one obtains the following relation

$$
\alpha=22.3210 Q^{-1.6} \ln Q-63.2414 Q^{-1.6}-1.0011
$$

The optimum value for $m$ is obtained through trial and error. Figure 4.7 (b) illustrates the estimated fit to the data with a total error of $1.4472 \mathbf{e}-05$. However, one should be aware of the limitations associated with Equation 4.7. As travel-time increases with an increasing receiver depth, hence increasing absorption, the curvature or "kink" observed in Figure 4.7 seemingly flattens and shifts towards increasing $Q$ values. Thus, one would need to exclude 
the lower $Q$ values in order to apply Equation 4.7. Plotting $\alpha$ against $Q$ for $z=630 \mathrm{~m}$ and $z=1230 m$ would require exclusion of $Q$ values lower than 50 and 100 respectively (illustrated in Figures 4.8 and 4.9). Minimising and obtaining the solution to Equation 4.7 yields the following

$$
\alpha=112.7576 Q^{-1.6} \ln Q-423.1553 Q^{-1.6}-1.0096
$$

and

$$
\alpha=291.5000 Q^{-1.6} \ln Q-1293.700 Q^{-1.6}-1.0000
$$

for $z=630 m$ and $z=1230 m$ respectively. The total error between data and estimated fit for $z=630 m$ is equal to 0.0005 and equal to $1.4286 e-06$ for $z=1230 m$.

In contrast to Figure 4.7 (a), with increasing absorption due to an increasing receiver depth, Equation 4.7 fails to capture or estimate the data $\left(\begin{array}{lll}\alpha & Q\end{array}\right)$ in its entirety. For $z=630 m$ and $z=1230 m$, Equation 4.7, only captures or provides an estimate to a portion of the data, as evident in Figures 4.8 and 4.9. Hence, Equation 4.7 fails to provide a robust mathematical description between $\alpha$ and $Q$ for all possible model scenarios (such as varying receiver depth values). Furthermore, one can not easily invert Equation 4.7 in order to estimate $Q$ values from $\alpha$, thus limiting the probable application(s) associated with absorption and the Lipschitz exponent $\alpha$. It should be stated that Equation 4.7 simply provides a mathematical estimate to the data, lacking any meaningful physical information between the two parameters. 


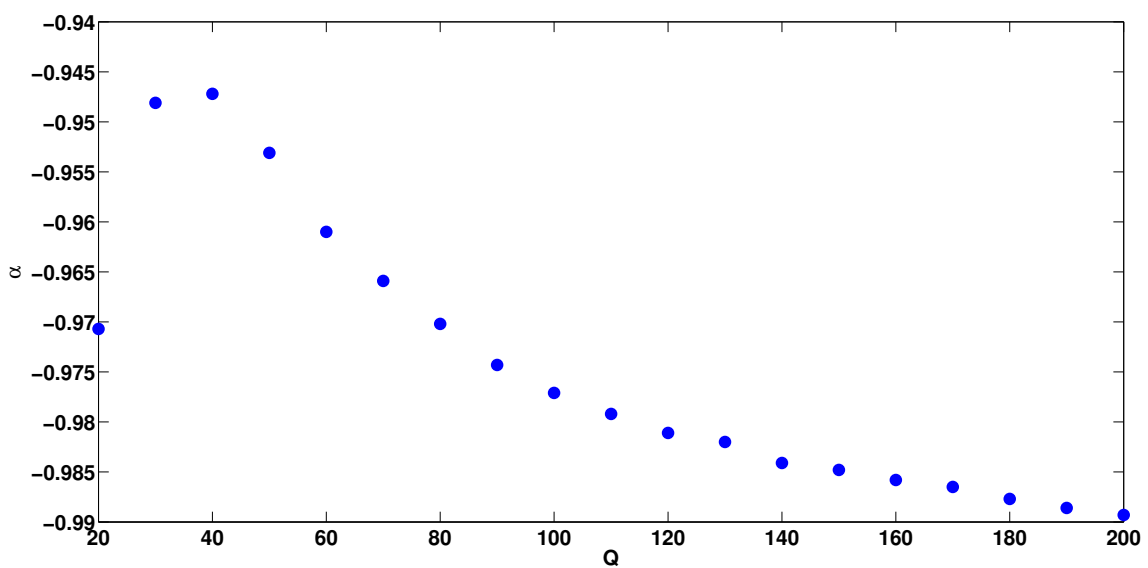

(a)

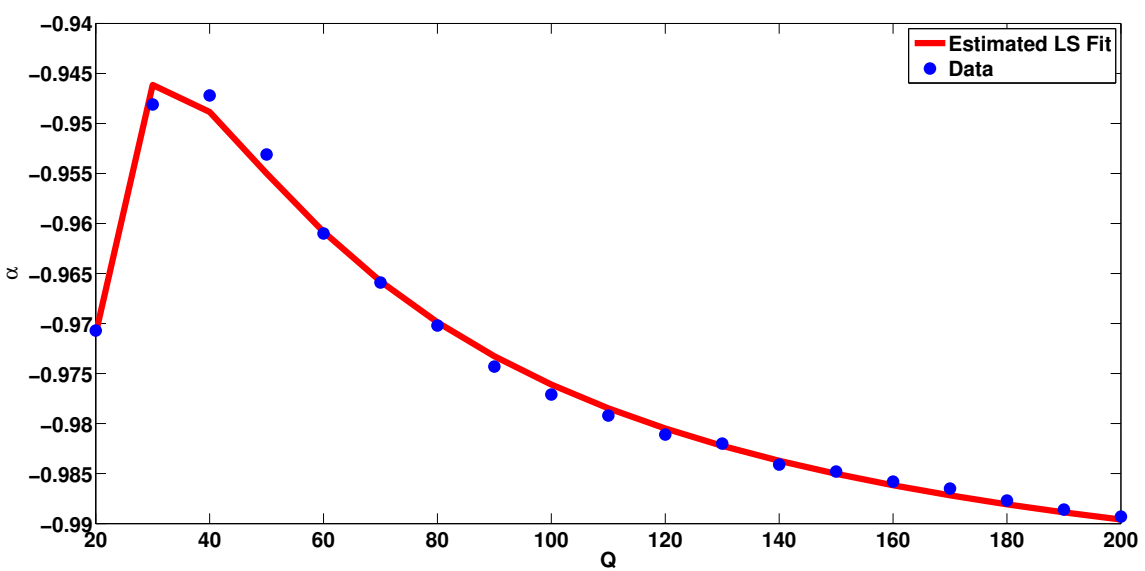

(b)

Figure 4.7: (a) Plot of $\alpha$ vs $Q$ for $z=270 m$ (b) Estimated Fit. 


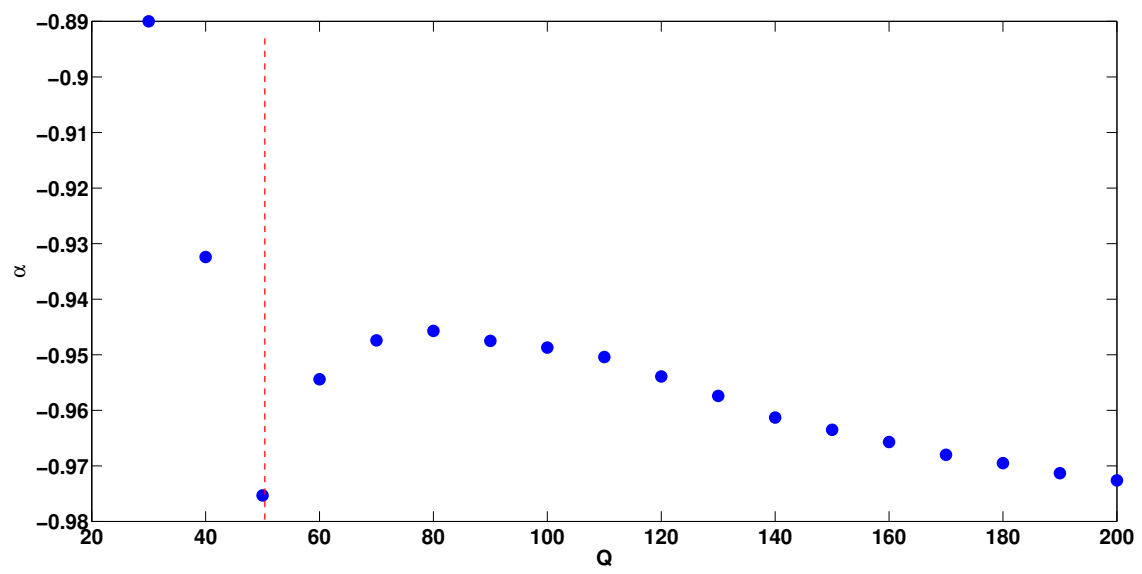

(a)

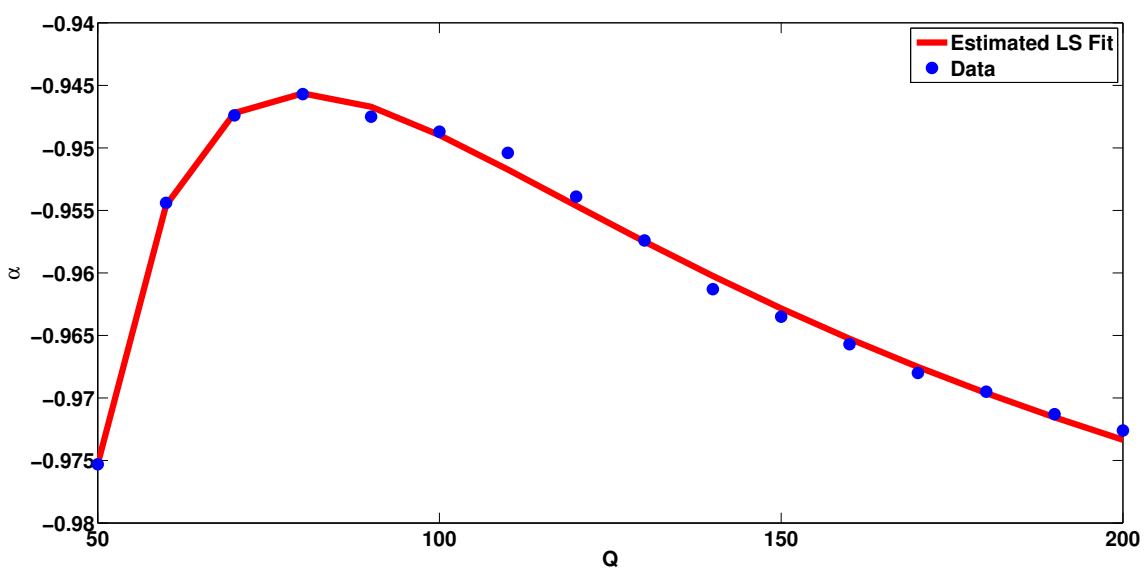

(b)

Figure 4.8: (a) Plot of $\alpha$ vs $Q$ for $z=630 m$ (b) Estimated Fit. In order to fit the data and reduce the corresponding errors between the fit and data points, the $\alpha$ values preceding the dashed line in (a) are excluded. 


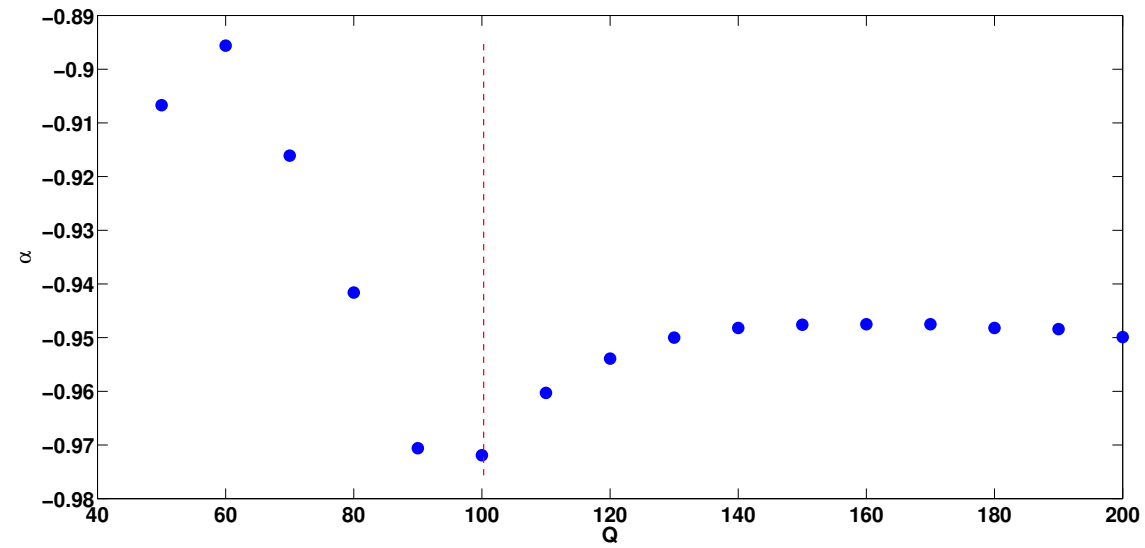

(a)

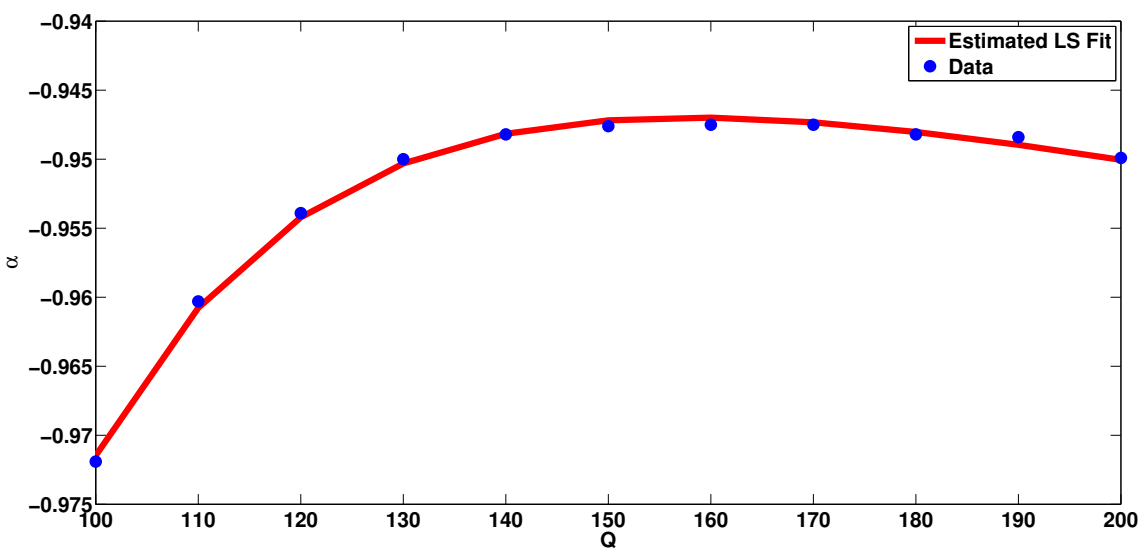

(b)

Figure 4.9: (a) Plot of $\alpha$ vs $Q$ for $z=1230 m$ (b) Estimated Fit. Similar to Figure 4.8 (a) the $\alpha$ values preceding the dashed line are excluded in order to improve the estimated fit to data.

\subsubsection{The relationship between smoothness, $\sigma$, and $Q$}

Given the limitations associated with the mathematical function mapping $\alpha$ to $Q$, one might consider the possible relation between the third parameter $\sigma$ and $Q$. Contrary to $\alpha$, plotting $\sigma$ against $Q$ values given in Table 4.4 reveals a relatively trivial power law type relation between the two variables. As illustrated in Figure 4.10, one could easily estimate a fit to the given data by the following relation

$$
\sigma=b_{1} Q^{b_{2}}
$$


For exponentially related data, one must linearise the problem and subsequently minimise by using the least squares method. Linearising Equation 4.14 yields the following expression

$$
\ln \sigma=\ln b_{1}+b_{2} \ln Q
$$

Hence, by minimising one obtains the following solutions

$$
b_{1}=\exp \left(\frac{\sum_{i=1}^{n}\left(\ln Q_{i}\right)^{2} \sum_{i=1}^{n} \ln \sigma_{i}-\sum_{i=1}^{n} \ln Q_{i} \ln \sigma_{i} \sum_{i=1}^{n} \ln Q_{i}}{n\left(\sum_{i=1}^{n}\left(\ln Q_{i}\right)^{2}\right)-\left(\sum_{i=1}^{n} \ln Q_{i}\right)^{2}}\right)
$$

and

$$
b_{2}=\frac{n \sum_{i=1}^{n} \ln Q_{i} \ln \sigma_{i}-\sum_{i=1}^{n} \ln Q_{i} \sum_{i=1}^{n} \ln \sigma_{i}}{n\left(\sum_{i=1}^{n}\left(\ln Q_{i}\right)^{2}\right)-\left(\sum_{i=1}^{n} \ln Q_{i}\right)^{2}} .
$$

Plotting $\sigma$ against the logarithm of $Q$ (figure 4.10 (b)), one could re-write Equation 4.14 as

$$
\sigma=b_{1}^{\prime} \ln Q^{b_{2}^{\prime}}
$$

Subsequently minimising yields the following solution

$$
b_{1}^{\prime}=\exp \left(\frac{\sum_{i=1}^{n}\left(\ln \left(\ln Q_{i}\right)\right)^{2} \sum_{i=1}^{n} \ln \sigma_{i}-\sum_{i=1}^{n} \ln \left(\ln Q_{i}\right) \ln \sigma_{i} \sum_{i=1}^{n} \ln \left(\ln Q_{i}\right)}{n\left(\sum_{i=1}^{n}\left(\ln \left(\ln Q_{i}\right)\right)^{2}\right)-\left(\sum_{i=1}^{n} \ln \left(\ln Q_{i}\right)\right)^{2}}\right)
$$

and

$$
b_{2}^{\prime}=\frac{n \sum_{i=1}^{n} \ln \left(\ln Q_{i}\right) \ln \sigma_{i}-\sum_{i=1}^{n} \ln \left(\ln Q_{i}\right) \sum_{i=1}^{n} \ln \sigma_{i}}{n\left(\sum_{i=1}^{n}\left(\ln \left(\ln Q_{i}\right)\right)^{2}\right)-\left(\sum_{i=1}^{n} \ln \left(\ln Q_{i}\right)\right)^{2}} .
$$

Additionally, one could improve the fit or estimation by applying additional weight on estimation points with lower error values relative to the data points. Referred to as the weighted least squares method, an estimation to data is obtained based on the following mathematical expression

$$
\min \sum_{i=1}^{n} \frac{\left[\ln \sigma_{i}-\left(\ln b_{1}+b_{2} \ln Q_{i}\right)\right]^{2}}{\left(\sigma_{i}^{\prime}\right)^{2}}
$$


where $\sigma_{i}^{\prime}$ is the standard deviation of the $i$ th observation. Due to the limiting fact that our data consists of a single observation, the error between the least squares fit (without weights) and data points has been assigned as weights in the weighted least squares estimation.

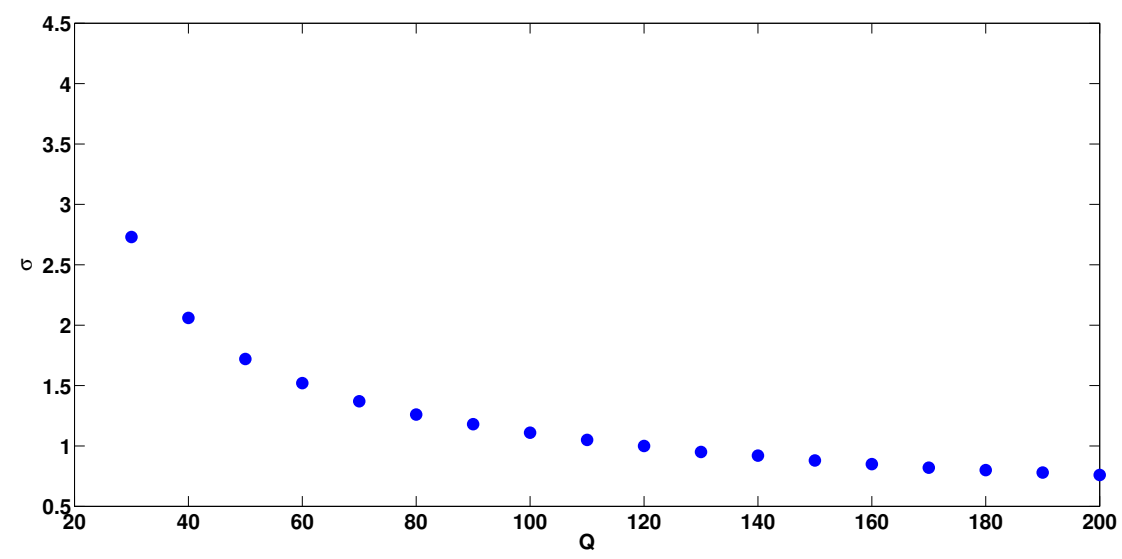

(a)

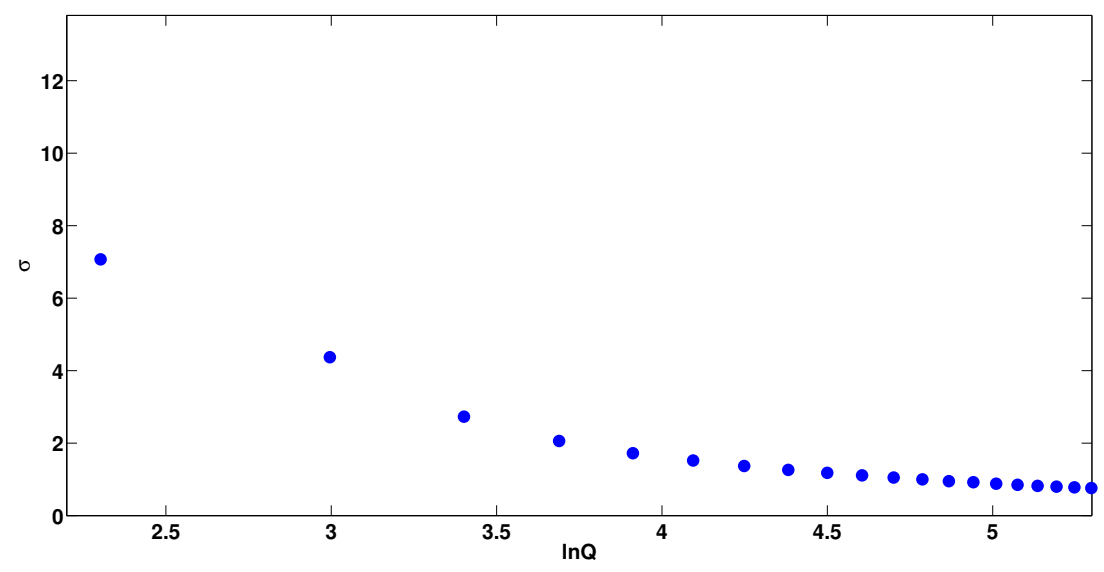

(b)

Figure 4.10: Plot of (a) $\sigma$ vs $Q$ (b) $\sigma$ vs $\ln Q$ for $z=270 m$.

Figure 4.10 illustrates the expected trend, such that increasing absorption broadens a given pulse, hence resulting in increasing $\sigma$ values. Compared to the least squares approximation, the weighted least squares method slightly overestimates the given data. However, one could improve the weighted least squares approximation by implementing an iterative method such that the weights $\left(\sigma_{i}^{\prime}\right)$ are updated at each step. The total error $\left(E_{i}\right)$ between 
the data and estimated least squares and the weighted least squares fit is 0.7491 and 1.5478 respectively. Additionally, plotting $\sigma$ against $\ln Q$ and subsequently minimising yields the most accurate fit with a total error of 0.1825 . The mathematical relation mapping $\sigma$ and $Q$, for a receiver located at $z=270 m$, is given by

$$
\sigma=29.1085 Q^{-0.7015}
$$

and

$$
\sigma=21.4660 Q^{-0.6390}
$$

for least squares and weighed least squares respectively. Additionally, the relation between $\sigma$ and $\ln Q$ for $z=270 m$ is given by

$$
\sigma=101.0296 \ln Q^{-2.9492}
$$




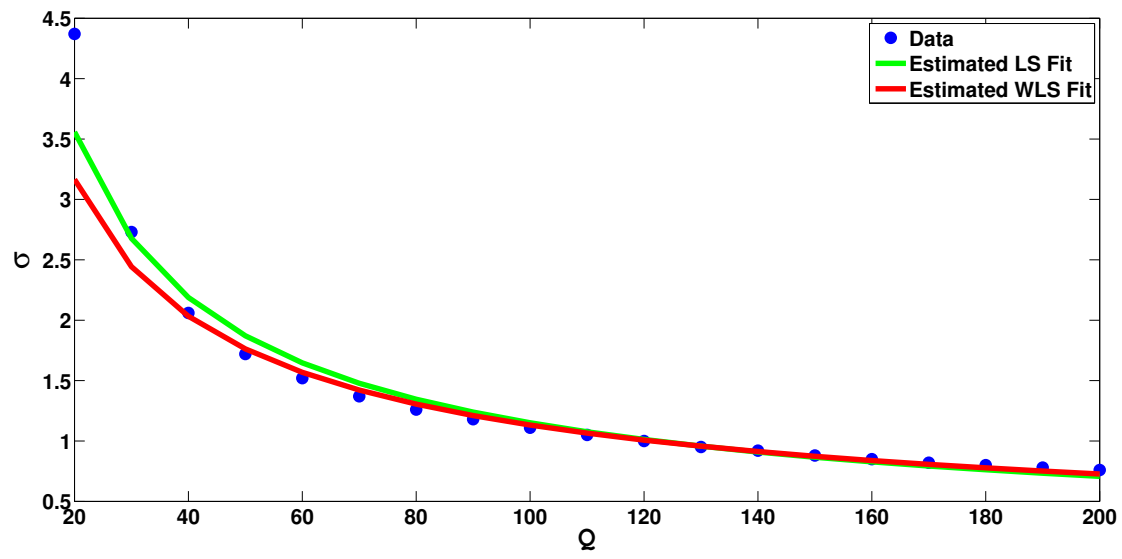

(a)

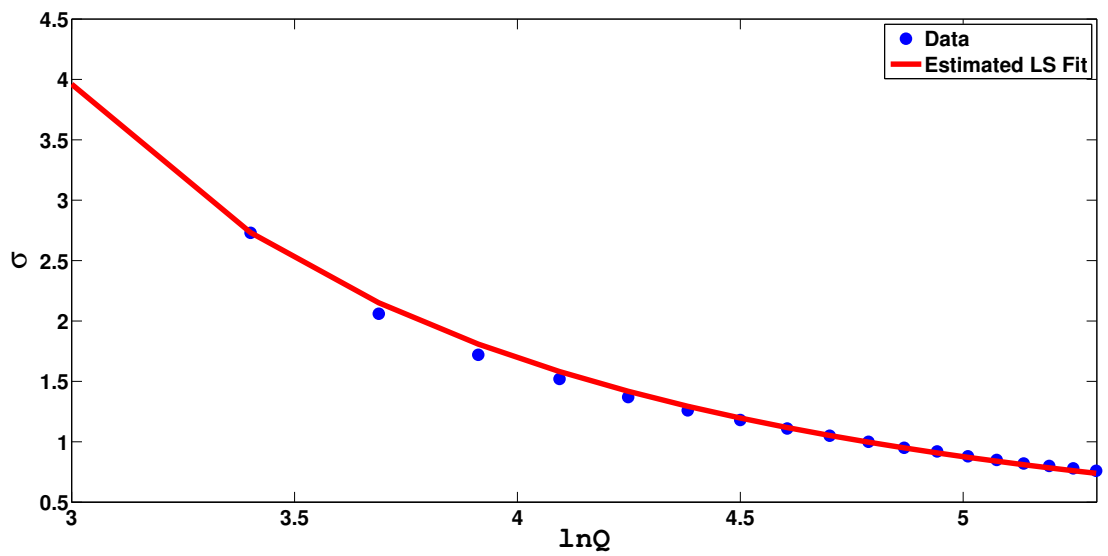

(b)

Figure 4.11: (a) Plot of $\sigma$ vs $Q \sigma$ vs $\ln Q$ (a) $\sigma$ vs $\ln Q$ for $z=270 m$. 


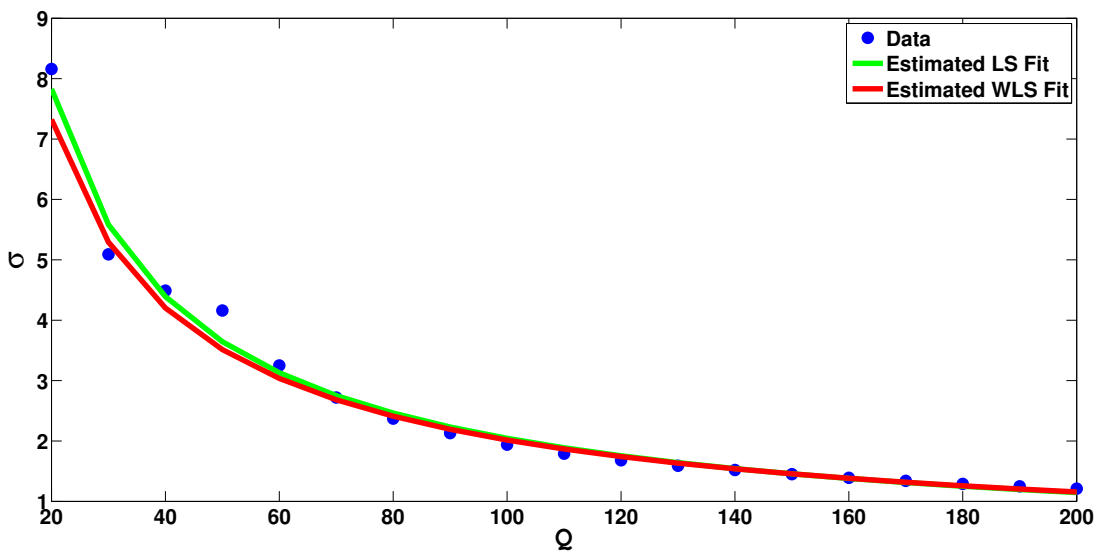

(a)

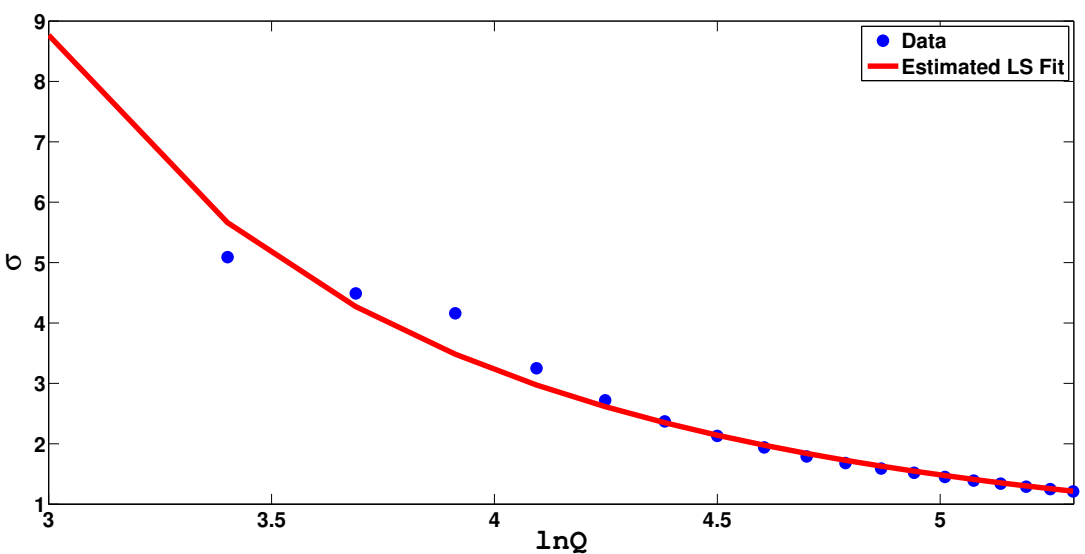

(b)

Figure 4.12: Plot of (a) $\sigma$ vs $Q$ (b) $\sigma$ vs $\ln Q$ for $z=630 \mathrm{~m}$. 


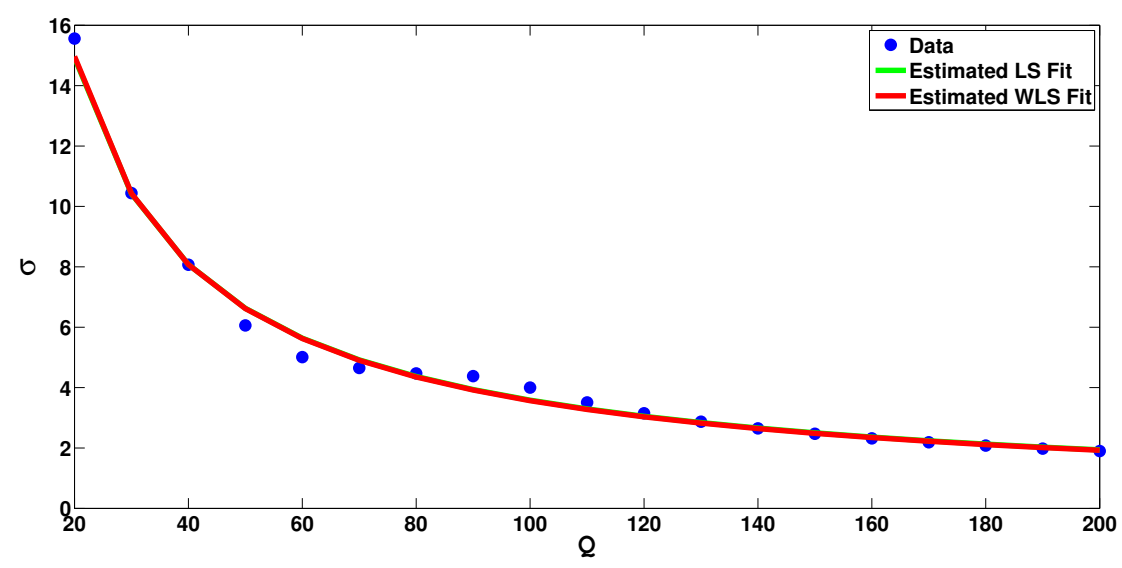

(a)

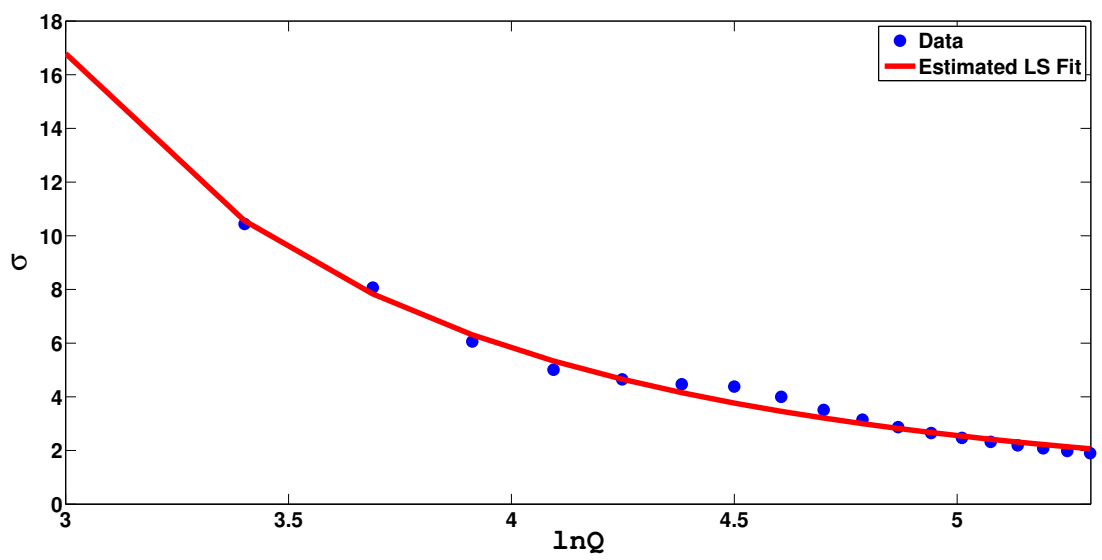

(b)

Figure 4.13: Plot of (a) $\sigma$ vs $Q$ (b) $\sigma$ vs $\ln Q$ for $z=1230 m$.

\begin{tabular}{|c|c|c|c|c|c|c|c|c|c|c|c|}
\hline & $z=270 m$ & $z=330 m$ & $\mathrm{z}=510 \mathrm{~m}$ & $\mathrm{z}=630 \mathrm{~m}$ & $z=750 \mathrm{~m}$ & $\mathrm{z}=870 \mathrm{~m}$ & $z=990 \mathrm{~m}$ & $z=1110 \mathrm{~m}$ & $z=1230 m$ & $\mathrm{z}=1350 \mathrm{~m}$ & $\mathrm{z}=1470 \mathrm{~m}$ \\
\hline$b_{1}$ & 29.10 & 46.77 & 70.60 & 95.38 & 123.59 & 149.83 & 169.59 & 198.46 & 212.26 & 225.05 & 246.50 \\
$\mathrm{~b}_{2}$ & -0.70 & -0.75 & -0.80 & -0.83 & -0.86 & -0.87 & -0.87 & -0.89 & -0.88 & -0.88 & -0.88 \\
$E_{i}$ & 0.74 & 0.67 & 0.43 & 0.69 & 0.77 & 0.88 & 1.07 & 1.26 & 1.63 & 2.21 & 2.66 \\
\hline
\end{tabular}

Table 4.5: Corresponding values mapping $\sigma$ to $Q$ using least squares.

\begin{tabular}{|c|c|c|c|c|c|c|c|c|c|c|c|}
\hline & $z=270 \mathrm{~m}$ & $z=330 \mathrm{~m}$ & $\mathrm{z}=510 \mathrm{~m}$ & $\mathrm{z}=630 \mathrm{~m}$ & $z=750 \mathrm{~m}$ & $\mathrm{z}=870 \mathrm{~m}$ & $z=990 \mathrm{~m}$ & $z=1110 \mathrm{~m}$ & $z=1230 \mathrm{~m}$ & $\mathrm{z}=1350 \mathrm{~m}$ & $\mathrm{z}=1470 \mathrm{~m}$ \\
\hline$b_{1}$ & 21.46 & 44.75 & 69.99 & 80.82 & 124.90 & 138.95 & 170.86 & 199.80 & 215.99 & 237.33 & 255.22 \\
$\mathrm{~b}_{2}$ & -0.63 & -0.74 & -0.80 & -0.80 & -0.86 & -0.86 & -0.88 & -0.89 & -0.89 & -0.89 & -0.89 \\
$E_{i}$ & 1.54 & 0.73 & 0.44 & 1.31 & 0.76 & 1.07 & 1.06 & 1.25 & 1.58 & 1.89 & 2.33 \\
\hline
\end{tabular}

Table 4.6: Corresponding values mapping $\sigma$ to $Q$ using weighted least squares. 


\begin{tabular}{|c|c|c|c|c|c|c|c|c|c|c|c|}
\hline & $z=270 m$ & $z=330 m$ & $\mathrm{z}=510 \mathrm{~m}$ & $\mathrm{z}=630 \mathrm{~m}$ & $z=750 \mathrm{~m}$ & $\mathrm{z}=870 \mathrm{~m}$ & $z=990 m$ & $z=1110 \mathrm{~m}$ & $z=1230 m$ & $\mathrm{z}=1350 \mathrm{~m}$ & $\mathrm{z}=1470 \mathrm{~m}$ \\
\hline$b_{1}^{\prime}$ & 101.02 & 173.10 & 280.81 & 397.25 & 535.14 & 662.00 & 752.38 & 900.01 & 957.36 & 1010.1 & 11163.0 \\
$\mathrm{~b}_{2}^{\prime}$ & -2.94 & -3.14 & -3.34 & -3.47 & -3.57 & -3.63 & -3.65 & -3.70 & -3.68 & -3.66 & -3.67 \\
$E_{i}$ & 0.18 & 0.87 & 1.05 & 1.33 & 1.59 & 2.66 & 2.44 & 3.13 & 2.89 & 2.50 & 2.89 \\
\hline
\end{tabular}

Table 4.7: Corresponding values mapping $\sigma$ to $\ln Q$.

Compared to the least squares method and based on the values provided in Tables 4.5 and 4.6, the total error associated with the weighted least squares method tends to improve with increasing receiver depth, hence increasing absorption. As illustrated in Figure 4.14, beyond receiver depth values greater than $z=990 m$, the weighted least squares estimation in comparison to the least squares method tends to improve the approximation to the given data. Nevertheless, all three methods yield a relatively accurate approximation to the data. Clearly, the addition of the third parameter $\sigma$ provides new insight and possibly a new avenue into the relation between the effects of absorption and applications of the continuous wavelet transform. Contrary to the Lipschitz exponent, one obtains a relatively trivial mathematical function relating $\sigma$ to the loss factor .

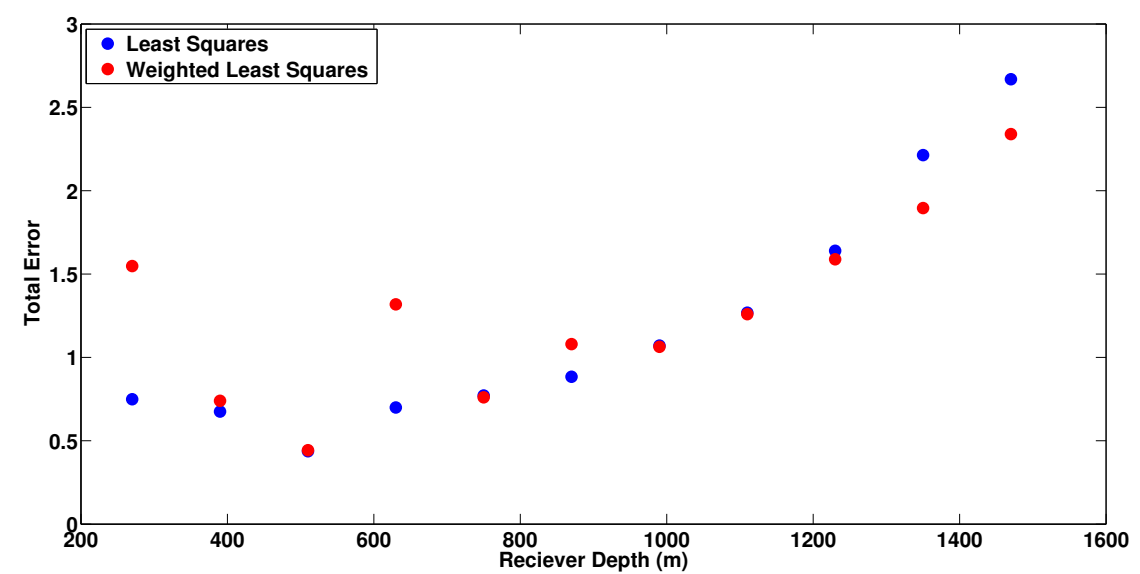

Figure 4.14: Comparison of total errors associated with least squares and weighted least squares approximation. 


\subsection{3 $Q$ estimation from MHZ model parameters}

In contrast to the Lipschitz exponent $(\alpha)$, one could easily invert Equations 4.14 and 4.18 in order to find a solution for $Q$. Hence, inverting, yields the following solutions

$$
Q=\left(\frac{\sigma}{b_{1}}\right)^{\frac{1}{b_{2}}}
$$

and

$$
Q=\exp \left[\left(\frac{\sigma}{b_{1}^{\prime}}\right)^{\frac{1}{b_{2}^{\prime}}}\right] .
$$

Using the $\sigma$ values corresponding to $z=270 \mathrm{~m}$ and respective coefficients given in Table 4.5, 4.6 and 4.7 the estimated $Q$ values obtained from Equation 4.25 provides a slightly more accurate estimate to true $Q$ values with lower absolute error values. However, as illustrated in Figure 4.16 (a) and (b), with increasing absorption, Equation 4.26 generally tends to approximate the true $Q$ values with higher degree of accuracy, thus lower absolute error values.

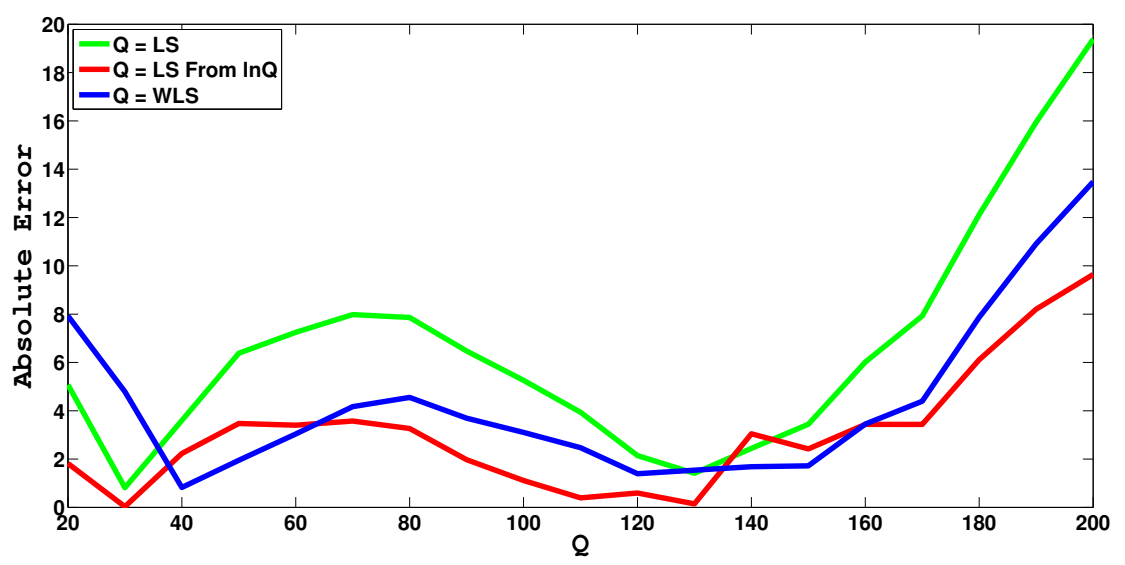

Figure 4.15: Comparison of absolute errors for $z=270 \mathrm{~m}$. 


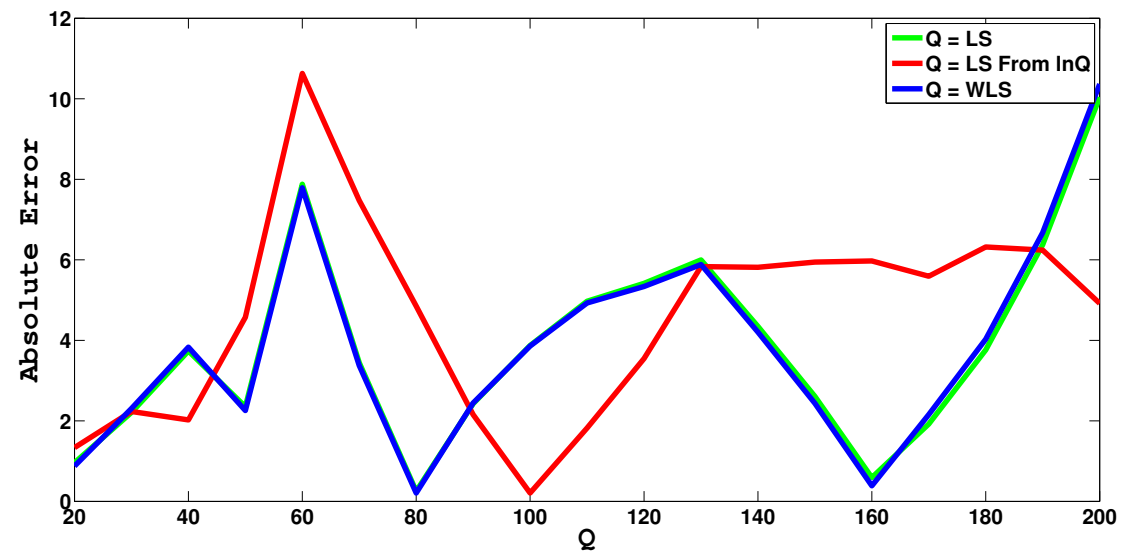

(a)

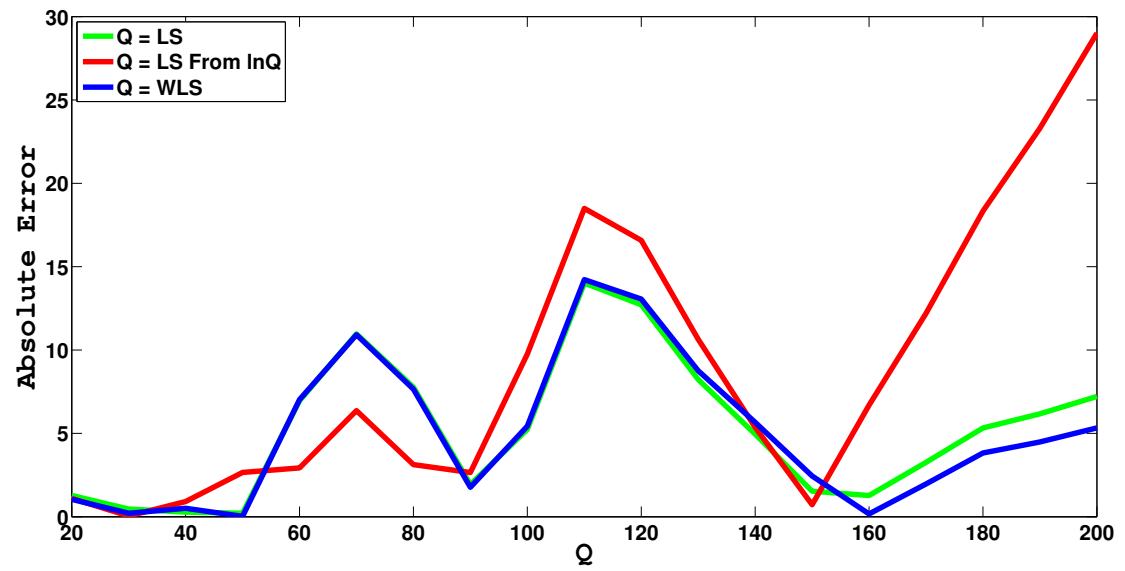

(b)

Figure 4.16: Comparison of absolute errors associated with least squares, weighted least squares and $\sigma \rightarrow \ln Q$ approximation for (a) $z=750 \mathrm{~m}$ (b) $z=1470 \mathrm{~m}$. 


\begin{tabular}{|c|c|c|c|}
\hline$z=270 m$ & Estimated $Q, L S$ & Estimated $Q, W L S$ & Estimated $Q$, from $\sigma \rightarrow \ln Q$ \\
\hline$Q=20$ & 14.9247 & 12.0737 & 18.1848 \\
\hline$Q=30$ & 29.1843 & 25.2116 & 30.0312 \\
\hline$Q=40$ & 43.5987 & 39.1736 & 42.2288 \\
\hline$Q=50$ & 56.3820 & 51.9510 & 53.4736 \\
\hline$Q=60$ & 67.2458 & 63.0393 & 63.4034 \\
\hline$Q=70$ & 77.9803 & 74.1701 & 73.5753 \\
\hline$Q=80$ & 87.8618 & 84.5507 & 83.2662 \\
\hline$Q=90$ & 96.4736 & 93.6919 & 91.9736 \\
\hline$Q=100$ & 105.2608 & 103.1019 & 101.1129 \\
\hline$Q=110$ & 113.9378 & 112.4699 & 110.3925 \\
\hline$Q=120$ & 122.1440 & 121.3941 & 119.4038 \\
\hline$Q=130$ & 131.4092 & 131.5407 & 129.8548 \\
\hline$Q=140$ & 137.5594 & 138.3152 & 136.9552 \\
\hline$Q=150$ & 146.5577 & 148.2800 & 147.5795 \\
\hline$Q=160$ & 153.9859 & 156.5516 & 156.5620 \\
\hline$Q=170$ & 162.0784 & 165.6073 & 166.5671 \\
\hline$Q=180$ & 167.8848 & 172.1323 & 173.8873 \\
\hline$Q=190$ & 174.0542 & 179.0895 & 181.7952 \\
\hline$Q=200$ & 180.6169 & 186.5198 & 190.3583 \\
\hline
\end{tabular}

Table 4.8: Estimated $Q$ values from 4.25 (using least squares and weighted least squared method) and 4.26 for $z=270 m$. 


\subsection{Towards application to field data}

\subsubsection{Noise}

It is generally a common practice to introduce noise within a given data in order to test the stability, effectiveness and robustness of the mathematical model. The extent of noise within given data is usually described by the signal-to-noise ratio (SNR). Mathematically the SNR value is given by the following expression

$$
S N R_{d B}=10 \log _{10}\left(\frac{E_{\text {Signal }}}{E_{\text {Noise }}}\right)
$$

where $\frac{E_{\text {Signal }}}{E_{\text {Noise }}}$ represents the energy ratio between the signal and background noise. Essentially the SNR is a mathematical expression for the ratio between the maximum possible signal value and power of distorting noise. Hence, based on Equation 4.27, typically lower SNR values indicate greater degree of noise within the data.

Figure 4.17 (a) and (b) represents direct arrivals corresponding to receiver location $z=630 \mathrm{~m}$ and $Q=20$ with $\mathrm{SNR}$ values of $18.01 \mathrm{~dB}$ and $2.27 \mathrm{~dB}$ respectively. Applying the continuous wavelet transform, measuring the corresponding modulus maxima values and subsequently minimising Equation 4.3 yields relatively accurate approximations to data containing normally distributed noise with SNR value of 18.01. Furthermore, the estimated $\alpha$ and $\sigma$ values are within close range to the estimated value corresponding to noise-free data. However, for data containing normally distributed noise with SNR value of $2.27 \mathrm{~dB}$ noise, the model seemingly breaks down, failing to provide an accurate estimation to $\alpha$ or $\sigma$.

\subsubsection{A Procedure for application to field data}

Based on the results from synthetic modelling and in order to estimate $Q$ values from zerooffset field data, we proceed by

1. Applying the continuous wavelet transform to seismic trace corresponding to direct arrival(s). 
2. Estimating the modulus maxima values, form the objective function based on Equation 4.2 and subsequently minimise (using the least squares method) in order to find the corresponding $\sigma$ values.

3. Subdividing the target medium into several layers and subsequently estimate the corresponding local $\sigma$ values for each layer by calculating the difference between the $\sigma$ values from the preceding layer and the target layer.

4. Inverting for $Q$ using Equation 4.25 and 4.26 for a range of $b_{1}, b_{2}$ and $b_{1}^{\prime}, b_{2}^{\prime}$ values.

5. Finally, comparing the obtained results to estimated $Q$ values from traditional methods, such as the spectral ration method. 


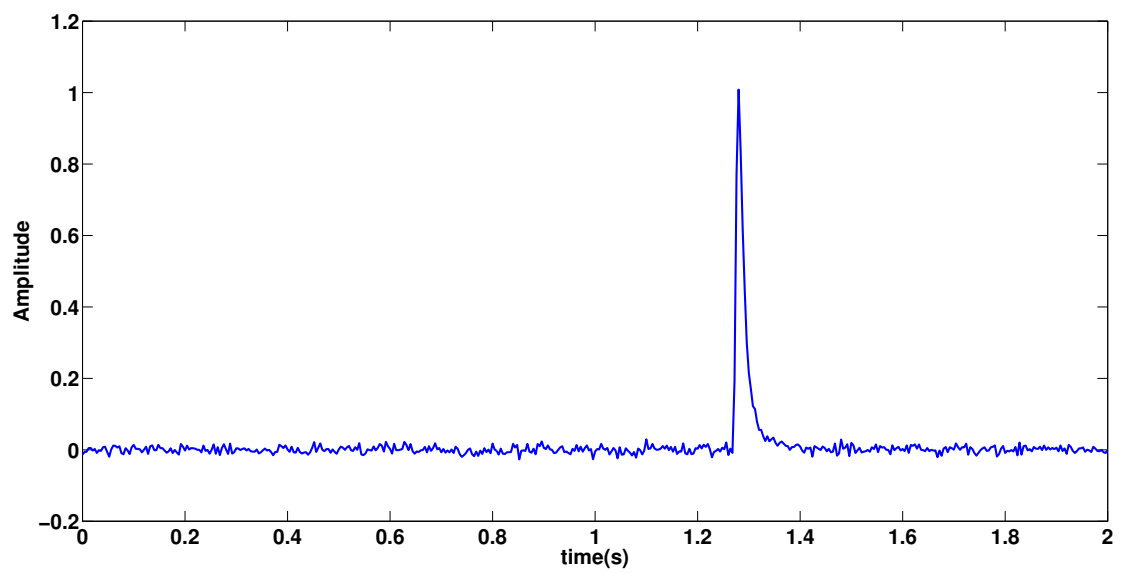

(a)

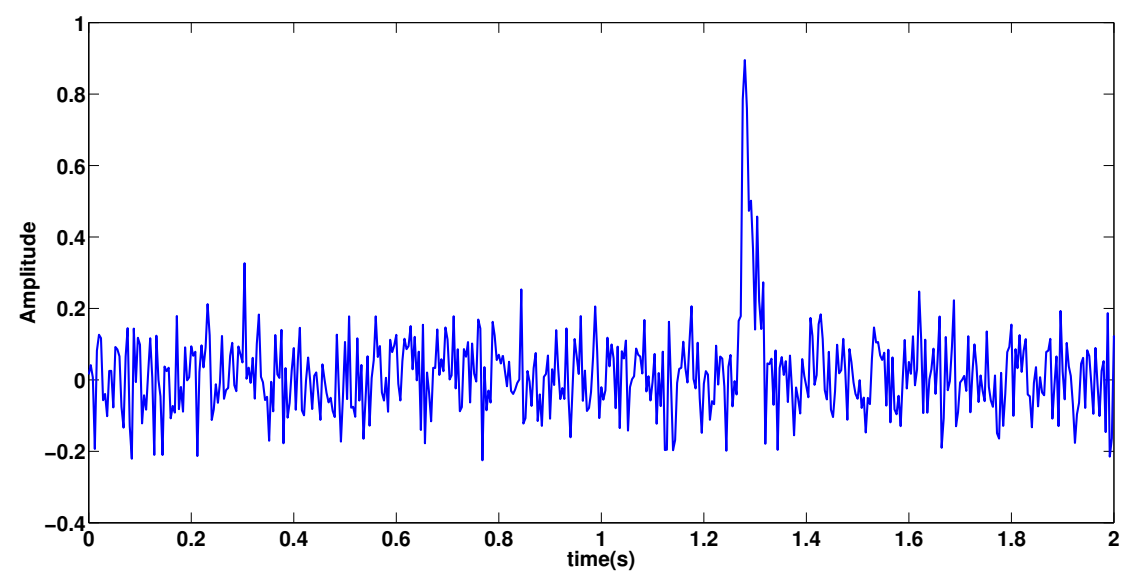

(b)

Figure 4.17: Direct arrivals corresponding to $z=630 \mathrm{~m}$ and $Q=20$. (a) Data containing normally distributed noise with SNR value of $18.01 \mathrm{~dB}$ noise (b) Data containing normally distributed noise with SNR value of $2.27 \mathrm{~dB}$. 


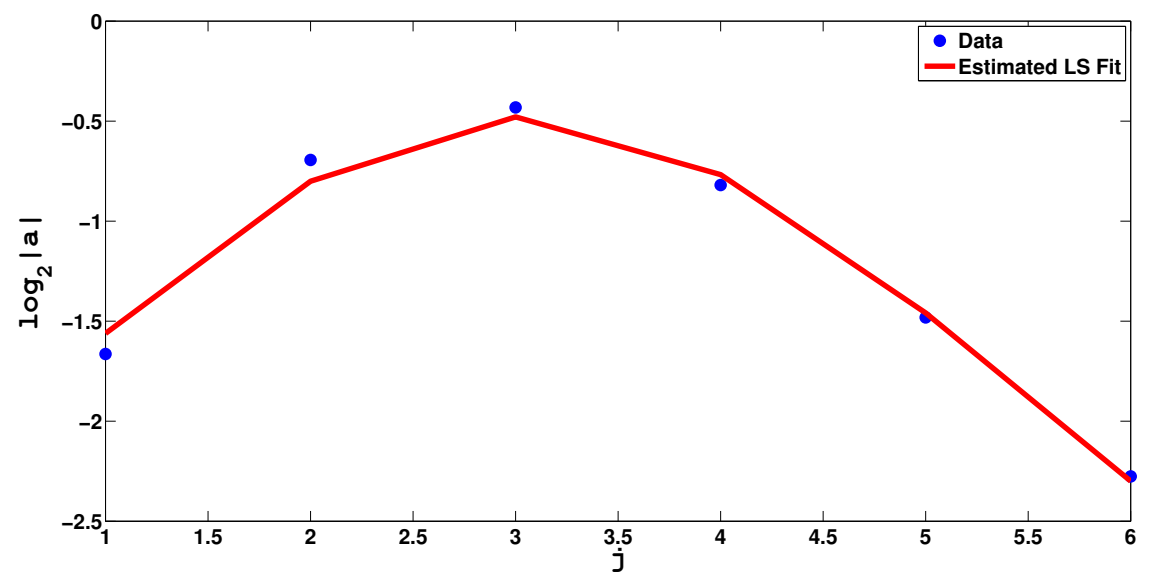

(a)

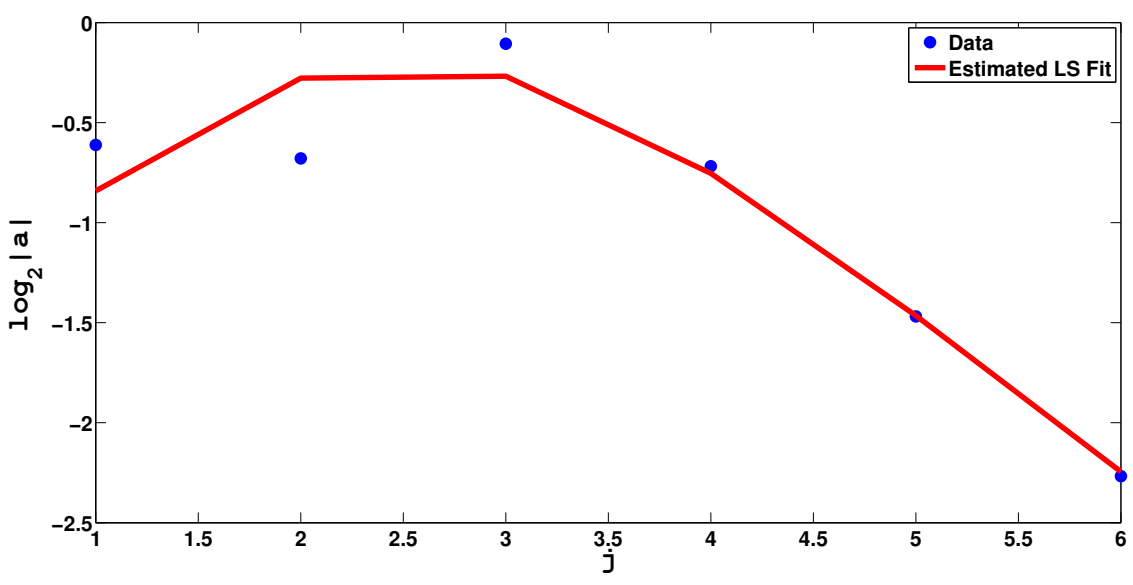

(b)

Figure 4.18: $\log _{2}\left|a_{i}\right|$ vs $j$ for direct arrival at $z=630 \mathrm{~m}$ and $Q=20$. a) Data containing normally distributed noise with SNR value of $18.01 \mathrm{~dB}$ noise (b) Data containing normally distributed noise with SNR value of $2.27 \mathrm{~dB}$. 


\section{Chapter 5}

\section{Application to the Ross Lake VSP field data set}

\subsection{Study area}

The validity, stability and ultimately viability of a mathematical model requires departure from a controlled setting (i.e. synthetic modelling), where input parameters are known, to an unknown, uncertain environment consisting of field data. The Ross Lake 3D VSP data, not only provides an opportunity to test, examine and analyse the behaviour of the modulus maxima on field data, but also an opportunity to study the empirical relation between $\alpha, \sigma$ and $Q$ obtained from synthetic modelling. In June 2003, the Ross Lake heavy oil field (located in south western Saskatchewan) was subject to a multi-offset VSP survey conducted by the CREWES project in conjunction with Husky Energy Inc. and Schlumberger Canada in order to study the relationship between rock properties and attenuation, AVO effect of the reservoir and to improve the characterisation of the Cretaceous Channel (Zhang, 2010).

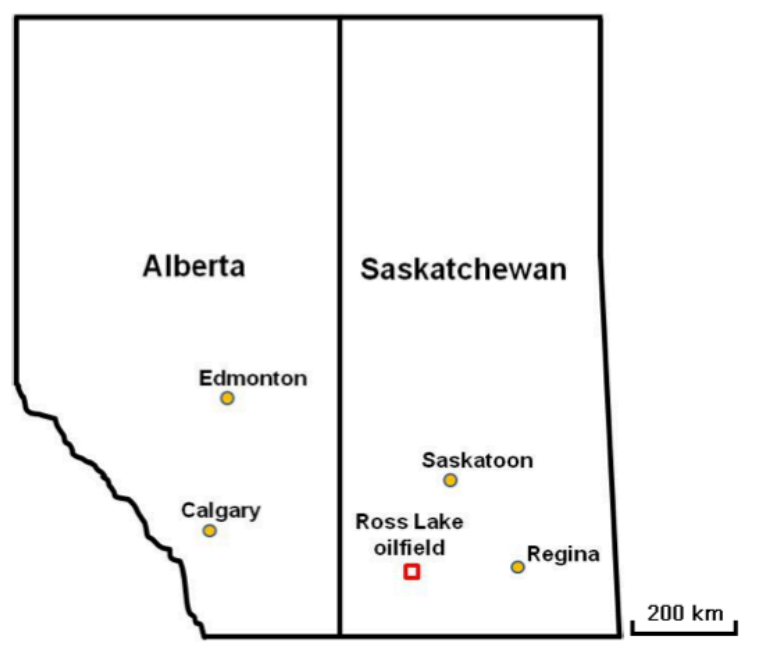

Figure 5.1: Ross Lake heavy oil field, (Zhang, 2010). 


\subsection{Data set}

The Ross Lake exploration target and producing reservoir was the Cretaceous Channel sand in the Dimmock Creek, member of the Mannville group, underlying the Jolie Fou formation of the Colorado group (Zhang, 2010). The Cantuar formation is subdivided into the McCloud, the Atlas and Dimmock Creek members, consisting of channel sands with a high degree of porosity and permeability. Furthermore, the Cantuar formation of the Mannville group mainly consists of sediments developed within the ancient valley system, carved into the upper Jurassic vanguard group (Christopher, 1974; Zhang, 2010). Figure 5.3 illustrates the regional stratigraphy of south western Saskatchewan.

The multi-offset VSP survey conducted by CREWES, Huskey Energy Inc. and Schlumberger Canada on June 2003, provided a detailed mapping of the Cantuar channel resevoir and further enhanced the interpretation of the 3C-3D seismic survey acquired in 2002 (Zhang, 2010). All conducted surveys utilised downhole five-level, three-component VSP tools with vertical and horizontal vibrator sources used for zero-offset VSP survey and vertical vibrator sources used for both far-offset and walkaway VSP surveys (Zhang, 2010).

For the zero-offset VSP survey, the horizontal component recorded reflected, transmitted and direct S-waves, whereas the vertical component mainly recorded the incoming P-waves. Hence, the horizontal and vertical components were used for processing SS and PP waves respectively (Zhang, 2010). Furthermore, for the vertical vibrator source, aligning the obtained zero-offset VSP data with first arrival time, estimating the downgoing P-waves by a 13-trace median filter and subsequently subtracting the downgoing P-wave from the wavefield yields an estimate for the upgoing P-wave (Zhang, 2010). Similarly, processing the zero-offset data obtained from the horizontal components yields an estimate for the upgoing and downgoing S-wave. Compared to the zero-offset VSP data, the far-offset VSP data requires a slightly different set of processing steps in order to determine the wave polarisation, clear upgoing and downgoing waves via data rotation and separate upgoing $\mathrm{P}$ and SV waves through 
time-variant data rotation (Zhang, 2010). However, by and large, the processing steps resemble that of zero-offset VSP data. Table 5.1 provides additional information regarding the zero-offset and far-offset VSP survey acquisition parameters.

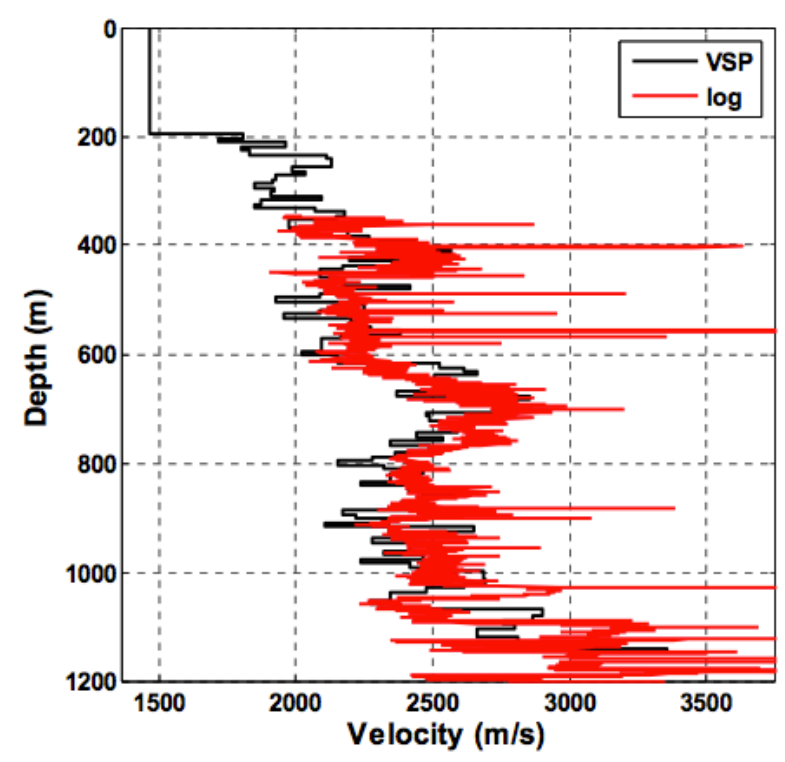

(a)

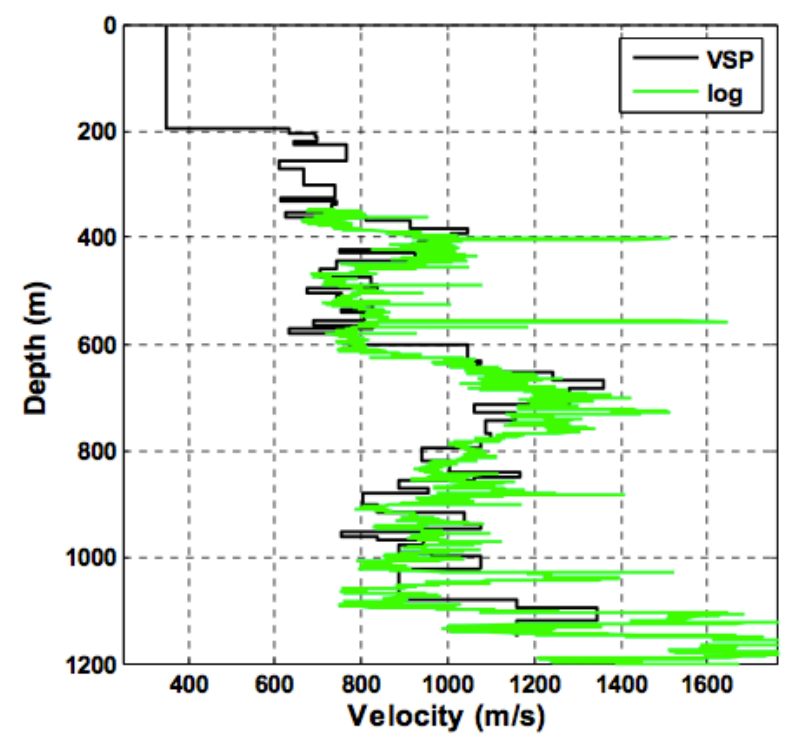

(b)

Figure 5.2: Estimated $\mathrm{P}$ and $\mathrm{S}$ wave velocities respectively, from zero-offset VSP first arrival time (Zhang, 2008). 


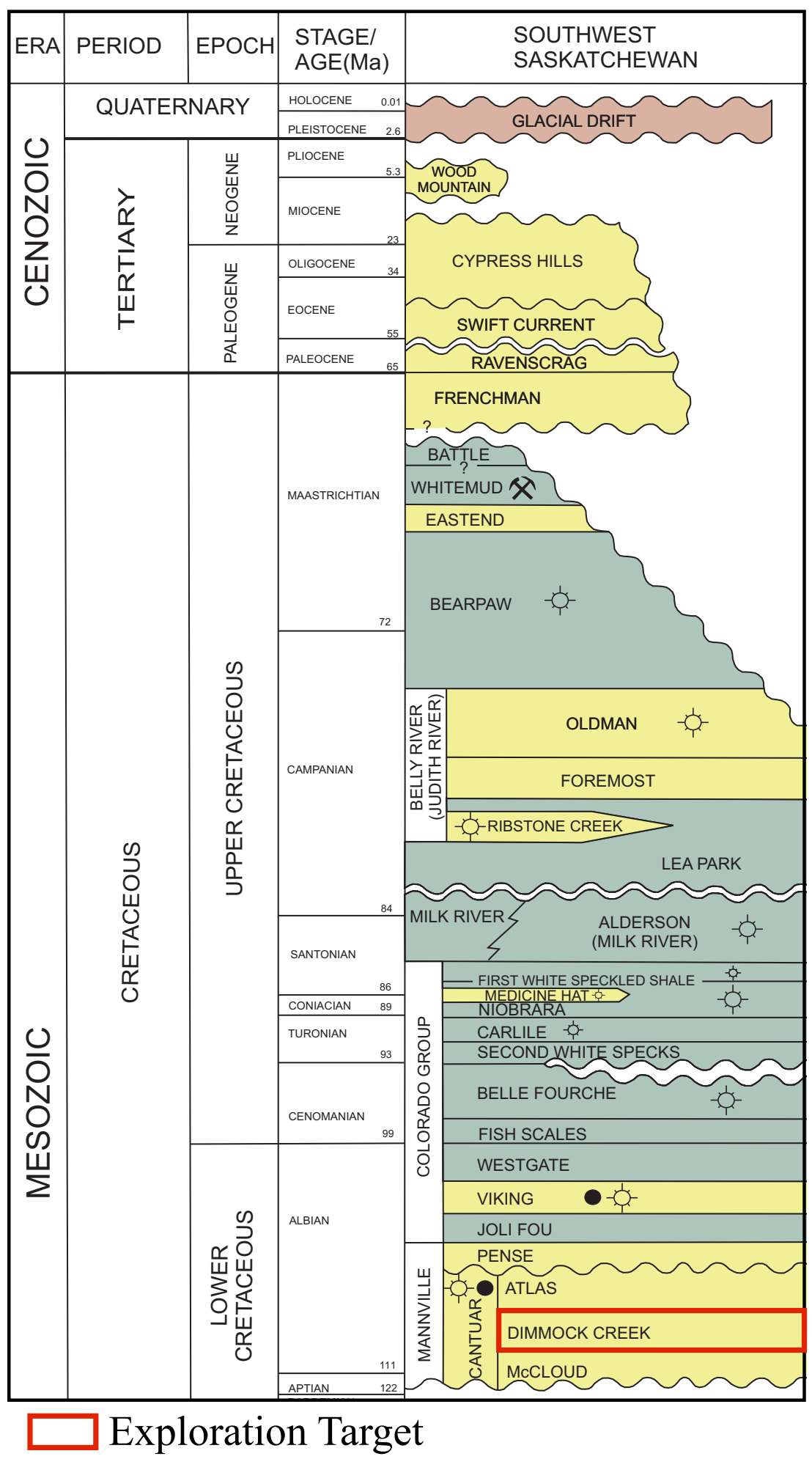

Figure 5.3: Regional stratigraphic chart in southwest Saskatchewan (Saskatchewan Industry and Resources, 2006). 


\begin{tabular}{|c|c|c|}
\hline Survey Type & Zero-offset VSP & Far-offset VSP \\
\hline Offset & $53.67 \mathrm{~m}$ & $399.12 \mathrm{~m}$ \\
Source Elevation & $856.10 \mathrm{~m}$ & $867.70 \mathrm{~m}$ \\
Source Azimuth & $16.30^{\circ}$ & $337.20^{\circ}$ \\
& Litton $315 \mathrm{P}$-vibe: & \\
& Sweep=8 - $180 \mathrm{~Hz}$, & Litton $315 \mathrm{P}$-vibe: \\
Source Type & IVI S-MINI vibe(inline): & Sweep=8-180 Hz \\
& Sweep= $5-100 \mathrm{~Hz} ; 12 \mathrm{~s}$ linear sweep & $197.50 \mathrm{~m}$ \\
Top Level & $197.50 \mathrm{~m}$ & $1165 \mathrm{~m}$ \\
Bottom Level & $1165 \mathrm{~m}$ & $7.50 \mathrm{~m}$ \\
Receiver Spacing & $7.50 \mathrm{~m}$ & \\
Reference Datum & $\mathrm{KB}=871.60 \mathrm{~m}$ & \\
\hline
\end{tabular}

Table 5.1: Acquistion parameters for the Ross Lake VSP survey (Zhang, 2008).

\subsection{1 $Q$ in the Ross Lake VSP data}

Whether the established empirical relation between $\sigma$ and $Q$, obtained from the synthetic data is applicable to the field data, hinges on the behaviour modulus maxima values applied on the field data. Figure 5.4 (a), represents the downgoing P-wave corresponding to the faroffset VSP survey. The offset value for this particular VSP survey is equal to $x=399.12 m$, with a total of 119 receivers spaced $7.5 \mathrm{~m}$ apart where the first receiver is located at a depth of $z=197.50 \mathrm{~m}$. Additionally, the corresponding VSP survey has a total number of 3001 samples with a sampling rate of $1 \mathrm{~ms}$.

Figure 5.4 (b), represents the trace of the direct P-wave arrivals corresponding to the 5th, 20th, 50th and 100th receiver. It is evident that the progressive amplitude decay implies a degree of absorption. Hence, based on theoretical properties and synthetic results, applying the continuous wavelet transform to the VSP data, calculating the corresponding modulus maxima values and plotting the logarithm against the wavelet scale should exhibit a certain degree of sensitivity to absorption within the data. Minimising the following equation,

$$
\log _{2}\left|a_{i}\right|-j=\log _{2}|A|+\frac{\alpha-1}{2} \log _{2}\left(\sigma^{2}+2^{2 j}\right)
$$

yields an estimate to $\alpha, \sigma$ and subsequently provides an approximation to the data. 


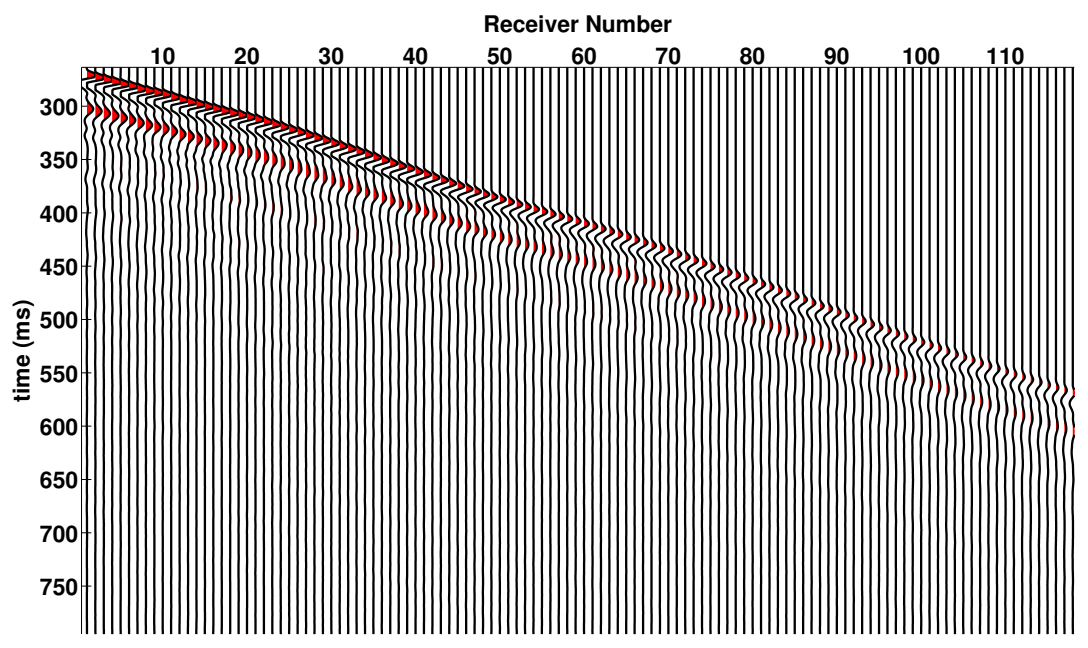

Figure 5.4: Downgoing P-wave corresponding to the far-offset VSP survey.

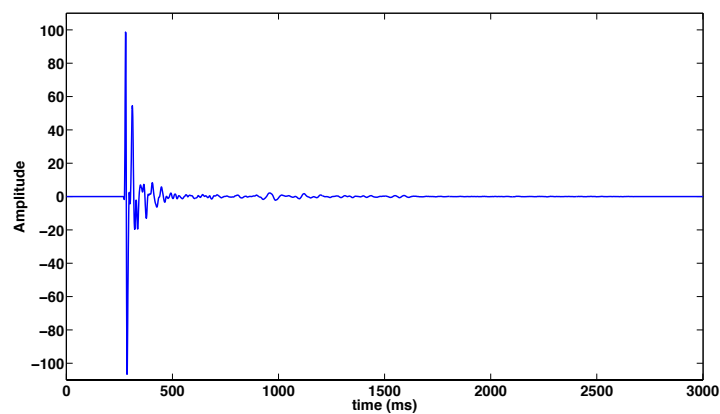

(a)

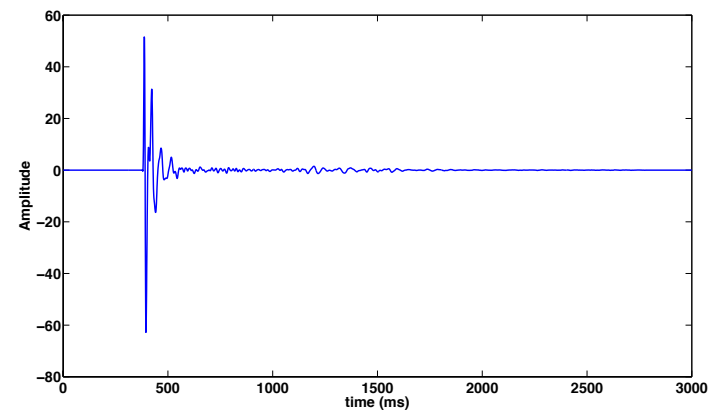

(c)

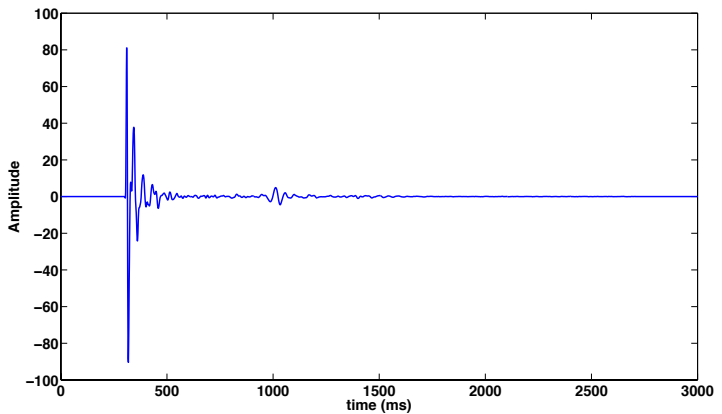

(b)

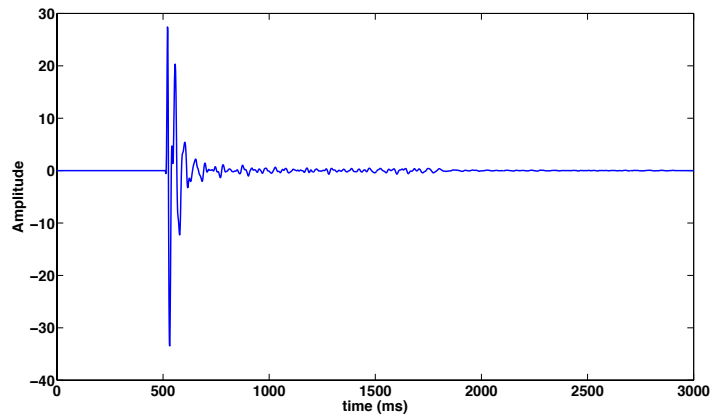

(d)

Figure 5.5: Trace of direct P-wave arrival corresponding to the (a) 5th, (b) 20th, (c) 50th and (d) 100th receiver. 


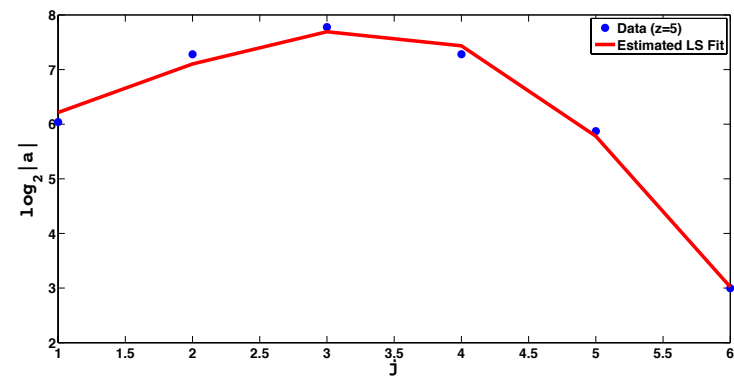

(a)

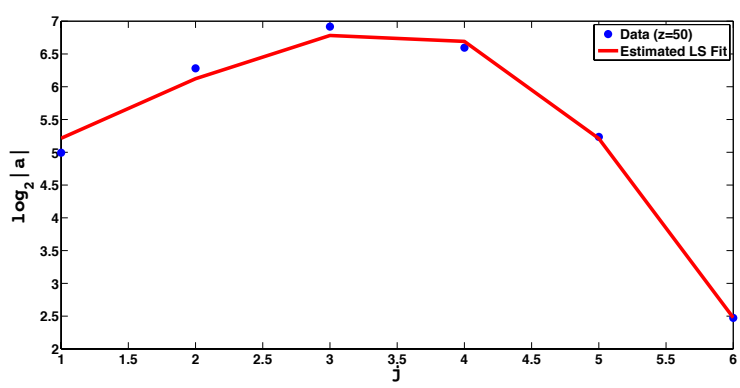

(c)

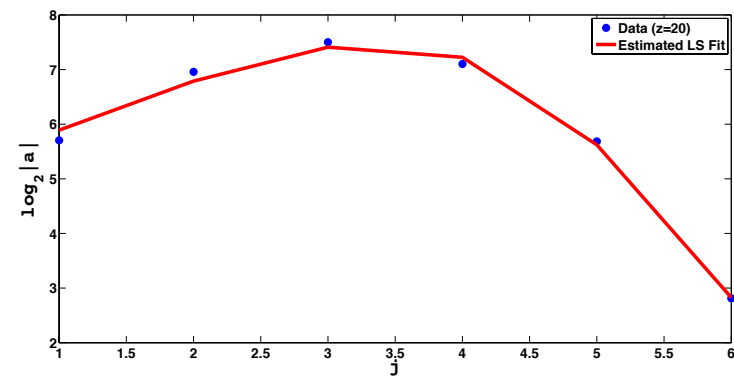

(b)

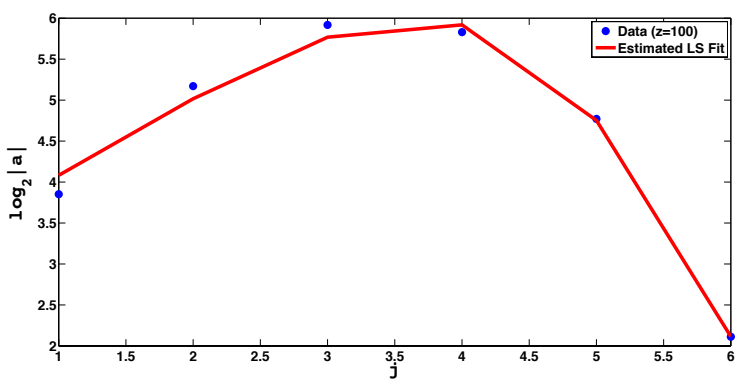

(d)

Figure 5.6: $\log _{2}\left|a_{i}\right|$ vs scale for P-wave corresponding to the (a) 5th, (b) 20th, (c) 50th and (d) 100th receiver respectively.

Figure 5.6, represents the logarithm of modulus maxima values against scale for the P-wave arrival corresponding to the 5th, 20th, 50th and 100th receiver. With increasing absorption, hence decay in amplitude, one could observe a gradual, progressive shift in curvature with increasing scale (from Figure 5.6 (a) to (d)). For field data, such gradual shift in curvature within data is encouraging, since it is via curvature that the model captures the effects of absorption, hence, it confirms and validates the sensitivity of the model to absorption. The total error between the least squares approximation and data corresponding to Figure 5.6 (a) to (d) is $0.1023,0.0909,0.1013$ and 0.1077 respectively. Thus, the relatively small error between the least squares fit to data yields an opportunity to estimate $Q$ based on the existing mathematical relation between $\sigma$ and the loss factor obtained from synthetic modelling. Figure 5.6, illustrates increasing $\sigma$ values with increasing receiver depth, hence 
an increase in absorption with increasing depth. The sudden "bump" or increase in $\sigma$ values roughly corresponding to the 50th to 70th receiver indicates a sudden increase in absorption. This could imply a decrease in velocity, thus resulting in a greater degree of absorption.

\subsubsection{Estimating Ross Lake MHZ parameters and $Q$}

Figure 5.7 represents the measured $\sigma$ values corresponding to the respective receiver. In order to estimate the corresponding $Q$ values, we need to measure the local $\sigma$ values. With the exception of the first $\sigma$ value, the remaining values represent the cumulative $Q$ effects from the previous layers. Mathematically, the cumulative effect could be represented as follows,

$$
\sigma_{m}=\sum_{n=1}^{m} b_{1} Q_{n}^{b_{2}}
$$

and

$$
\sigma_{m}=\sum_{n=1}^{m} b_{1}^{\prime} \ln Q_{n}^{b_{2}^{\prime}}
$$

where $m$ represents the corresponding layer. In order to exclude the cumulative $Q$ effects from the previous layers and measure the local $\sigma$ value(s), we deduct the two successive $\sigma$ values from each other. For example, in order to estimate the local $\sigma$ value corresponding to the second receiver, we deduct the first value from the second $\sigma$ value as follows,

$$
\sigma_{\text {local_2 }}=\sigma_{2}-\sigma_{1} \Longrightarrow \triangle \sigma_{2}=b_{1} Q_{2}^{b_{2}}+b_{1} Q_{1}^{b_{2}}-b_{1} Q_{1}^{b_{2}}=b_{1} Q_{2}^{b_{2}}
$$

Repeating the process provides an estimate to the local $\sigma$ values corresponding to each receiver. Figure 5.8, represents the estimated local $\sigma$ values corresponding to each receiver. Clearly, the highest value $\left(\triangle \sigma_{1}=18.2800\right)$ indicates a high degree of absorption within the first layer (from source to the first receiver). However, based on the Figure 5.8 the estimated local $\sigma$ values (excluding the first value) reveal negligible yet gradual absorption, with the maximum local $\sigma$ value equal to 0.8600 . As a result, the medium has been subdivided into six intervals or layers, hence measuring the average $Q$ value corresponding to each layer. Table 5.2 represents the $\sigma$ values corresponding to each layer. It should be noted that the $\sigma$ 
corresponding to the second layer is calculated by deducting the local $\sigma$ value corresponding to the first receiver from the $\sigma$ corresponding to the 34 th receiver (located at $z=450 \mathrm{~m}$ ). Hence the estimated local $\sigma$ value corresponds to the average $Q$ values from $z=200 \mathrm{~m}$ to $z=450 \mathrm{~m}$. The rest of the $\sigma$ values (corresponding to the remaining layers) are estimated in similar fashion.

\begin{tabular}{|c|c|}
\hline $\operatorname{Depth}(m)$ & Local $\sigma$ value \\
\hline $7-200$ & 18.2800 \\
$200-450$ & 1.3000 \\
$450-600$ & 2.4300 \\
$600-800$ & 1.4800 \\
$800-1000$ & 2.3600 \\
$1000-1165$ & 1.2400 \\
\hline
\end{tabular}

Table 5.2: Corresponding depth and local $\sigma$ values.

Thus far, based on synthetic modelling, the following two mathematical expressions

$$
Q=\left(\frac{\sigma}{b_{1}}\right)^{\frac{1}{b_{2}}}
$$

and

$$
Q=\exp \left[\left(\frac{\sigma}{b_{1}^{\prime}}\right)^{\frac{1}{b_{2}^{\prime}}}\right] .
$$

exists, where a power law function provides a mapping between $Q$ and $\sigma$. It should be noted that $b_{1}$ and $b_{2}$ were obtained using the least squares and the weighted least squares method. Table 1.2, provides the minimum, maximum and mid-level values obtained for each coefficient based on a wide range of tested synthetic models.

Applying the minimum and mid-range values given in Table 5.3 to Equation 5.5 and 5.6, yields unrealistic $Q$ values corresponding to the first layer, with minimum $Q$ values ranging from roughly 1.2859 to just under 15 . However, the remaining $Q$ values range from the high twenties to just under 270, a relatively acceptable range. Additionally, applying the maximum obtained values yields $Q$ estimates ranging from roughly 20 to $Q$ values slightly over 600. Figure 5.9, represented the estimated $Q$ values using Equations 5.5 and 5.6. For the minimum and mid-range values Equation 5.5 and 5.6 provide $Q$ values in relative close 
proximity. However, using the maximum values given in Table 5.3, Equation 5.6 seems to overestimate the measured $Q$ values.

\begin{tabular}{|c|c|c|c|}
\hline & Minimum Value & Mid-level Value & Maximum Value \\
\hline$b_{1}$ & 29.1085 & 149.8336 & 259.5581 \\
$b_{2}$ & -0.7015 & -0.8765 & -0.8787 \\
$b_{1 w}$ & 21.4660 & 138.9541 & 261.0325 \\
$b 2_{w}$ & -0.6390 & -0.8617 & -0.8807 \\
$b_{1}^{\prime}$ & 101.0296 & 662.0009 & 1176.3000 \\
$b_{2}^{\prime}$ & -2.9492 & -3.6388 & -3.6624 \\
\hline
\end{tabular}

Table 5.3: Maximum, medium-range and minimum coefficient values from synthetic modelling were $b_{1}, b_{2}$ is obtained from least squares method and $b_{1 w}, b_{2 w}$ is obtained from weighted least squares method.

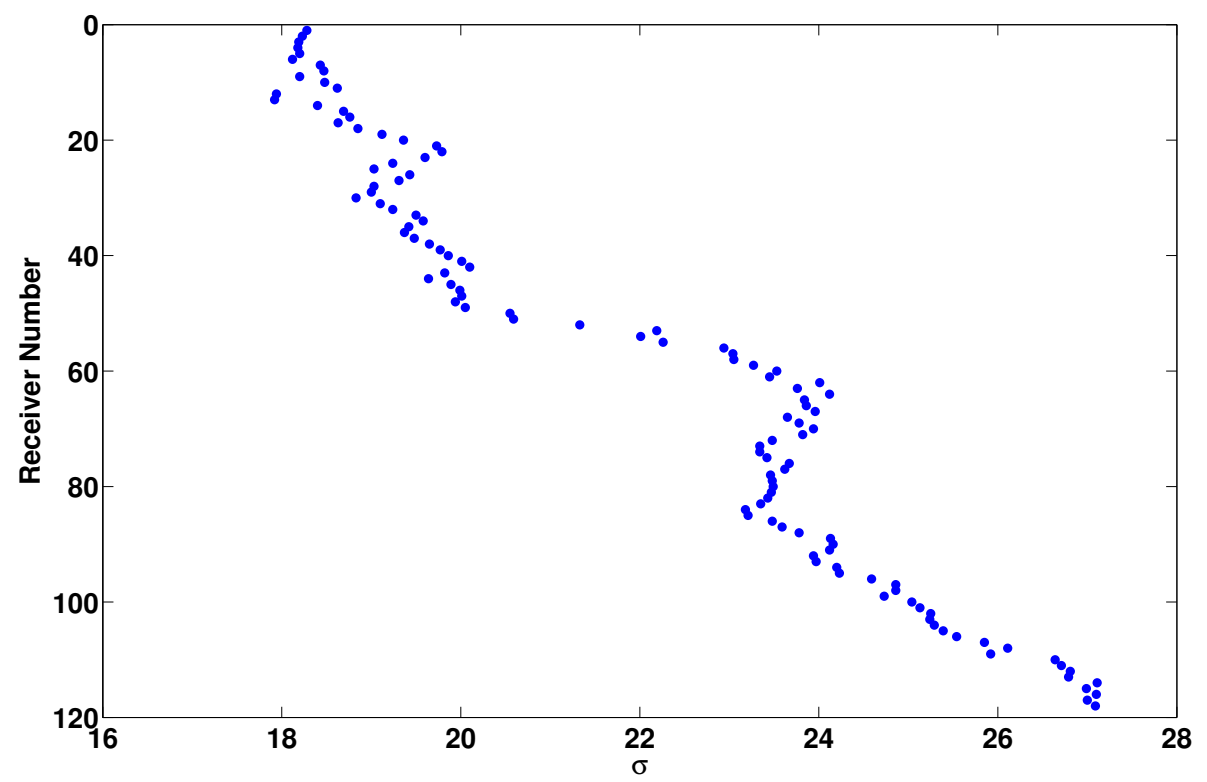

Figure 5.7: Plot of estimated $\sigma$ values against receiver number. 


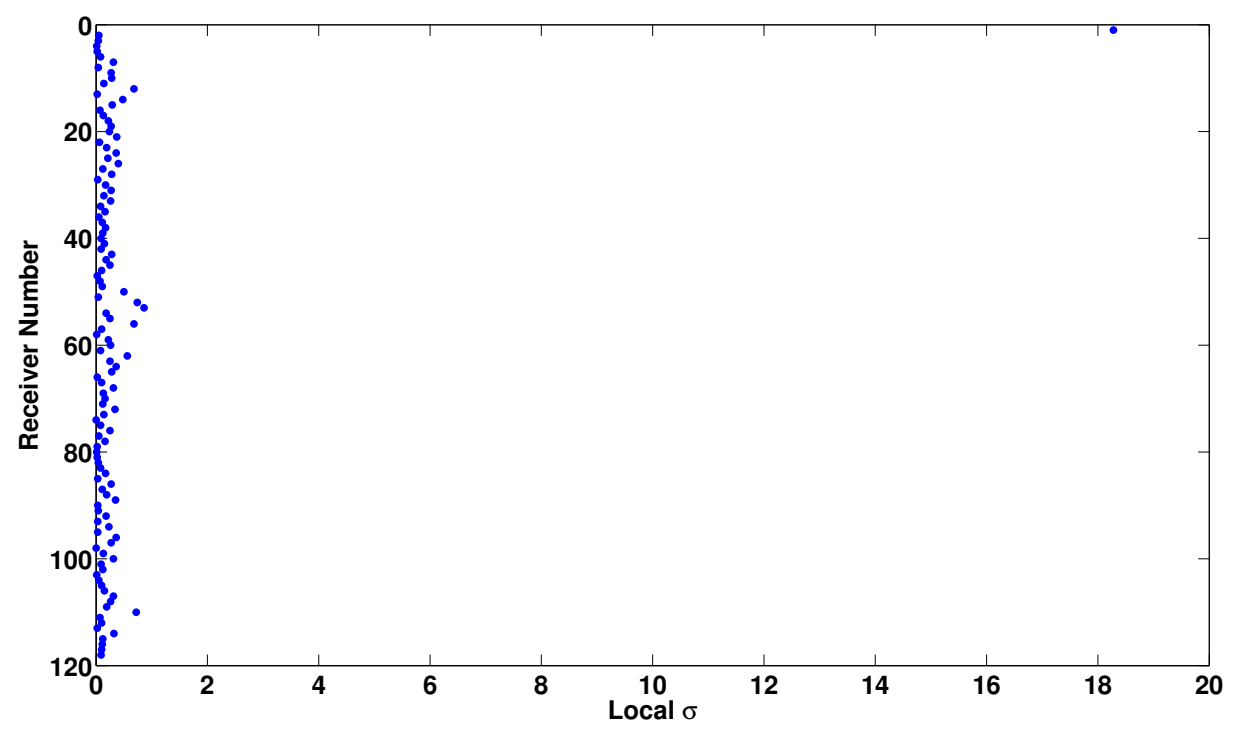

Figure 5.8: Plot of estimated local $\sigma$ values against receiver number.

In order to estimate and measure the attenuate factor $Q$, the most commonly used method or technique in the field of exploration geophysics is the spectral ratio method. The method utilises change in spectra at different depth levels in order to estimate the attenuation factor $Q$ (Haase and Stewart, 2003). Table 5.4, provides the estimated $Q_{p}$ values from downgoing P-wave obtained from the Ross Lake VSP data. From an interval of $z=200 \mathrm{~m}$ to $z=1000 \mathrm{~m}$ the $Q_{p}$ values estimated by $\mathrm{Xu}$ and Stewart (2004) generally reveals a downward trend in $Q_{p}$ values with a maximum value of $Q_{p}=197$ and minimum value of $Q_{p}=28$. Hence, for the most part, absorption seems to be increasing. For the deepest interval, $z=1000 \mathrm{~m}$ to $z=1165 m$, absorption seems to decrease with $Q_{p}$ value of 136 . The general trend for absorption (from $z=450 m-1165 m$ ) seems to be captured by the estimated $\sigma$ and $Q$ values, with the minimum and mid-level values provided in Table 5.3 yielding relatively accurate $Q$ values to the estimated values by using the spectral ratio method. 


\begin{tabular}{|c|c|c|}
\hline & $Q_{p}$ & $Q_{p}$ \\
\hline Depth & $(\mathrm{a})$ & $(\mathrm{b})$ \\
\hline $200-450 m$ & 197 & \\
$450-600 m$ & 28 & \\
$600-800 m$ & 51 & 67 \\
$800-1000 m$ & 46 & \\
$1000-1165 m$ & 136 & \\
\hline
\end{tabular}

Table 5.4: Estimated $Q_{p}$ values from downgoing P-wave were (a) and (b) represent the values obtained by Xu, Stewart (2004) and Haas (2003) respectively (Zhang and Stewart, 2006).

\subsubsection{Discussion}

In essence, the spectral ratio method employs a linear fit to the data (logratio of a amplitudes vs frequency), with the estimated slope of the fit yielding a linear mapping to $Q$. However, Figure 5.10 illustrates a major drawback associated with the spectral ratio method. Although a linear downward trend is used as a justification for a linear fit, one could clearly notice the degree of errors between the fit and data, which ultimately carried into the $Q$ estimate. However, without extensive error analysis it is difficult to comment on the accuracy on the method. Nevertheless, the spectral ratio method is the most commonly utilised method in the field of exploration geophysics in order to estimate the attenuation factor. Hence, new techniques and proposed methods should be measured against the spectral ratio method. Clearly, the estimated Q values using the continuous wavelet transform falls short compared to the estimated $Q$ values using the spectral ratio method. 


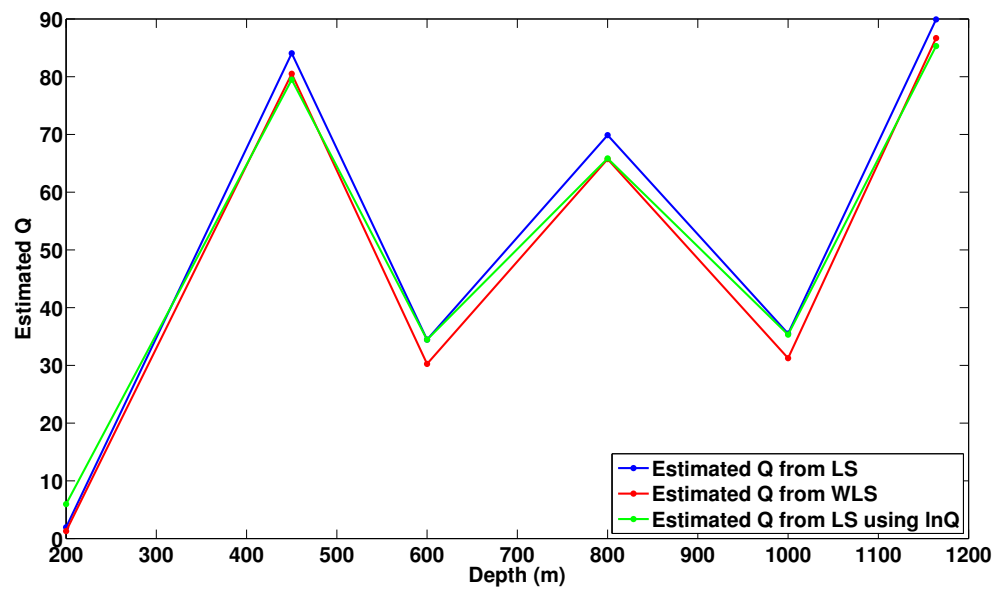

(a)

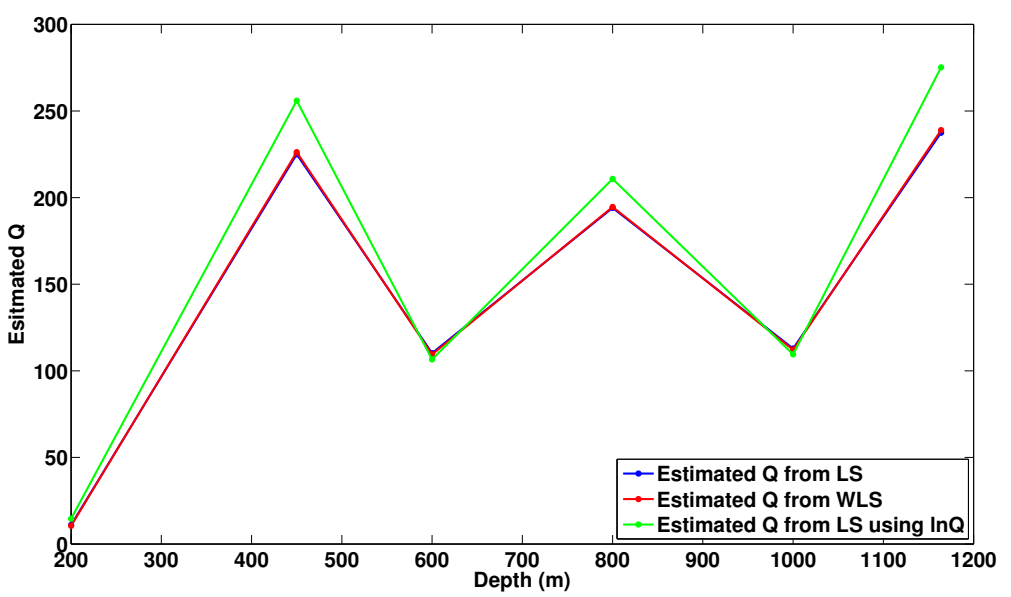

(b)

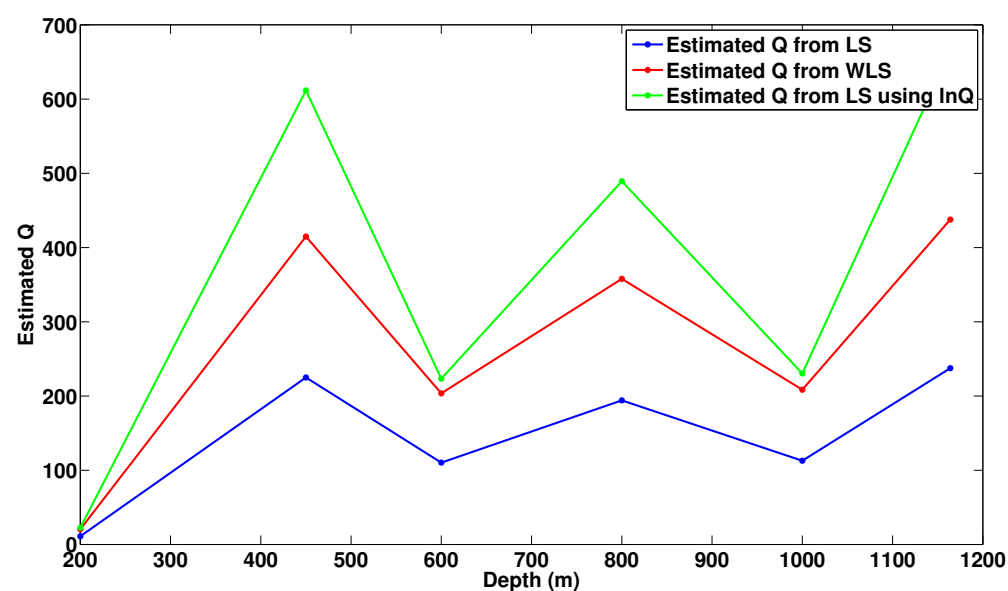

(c)

Figure 5.9: Estimated $Q$ values using Equation 5.5 and 5.6 with (a) Minimum obtained values given in Table 5.3 (b) Mid-level obtained values given in Table 5.3 (c) Maximum obtained values given in Table 5.3. 


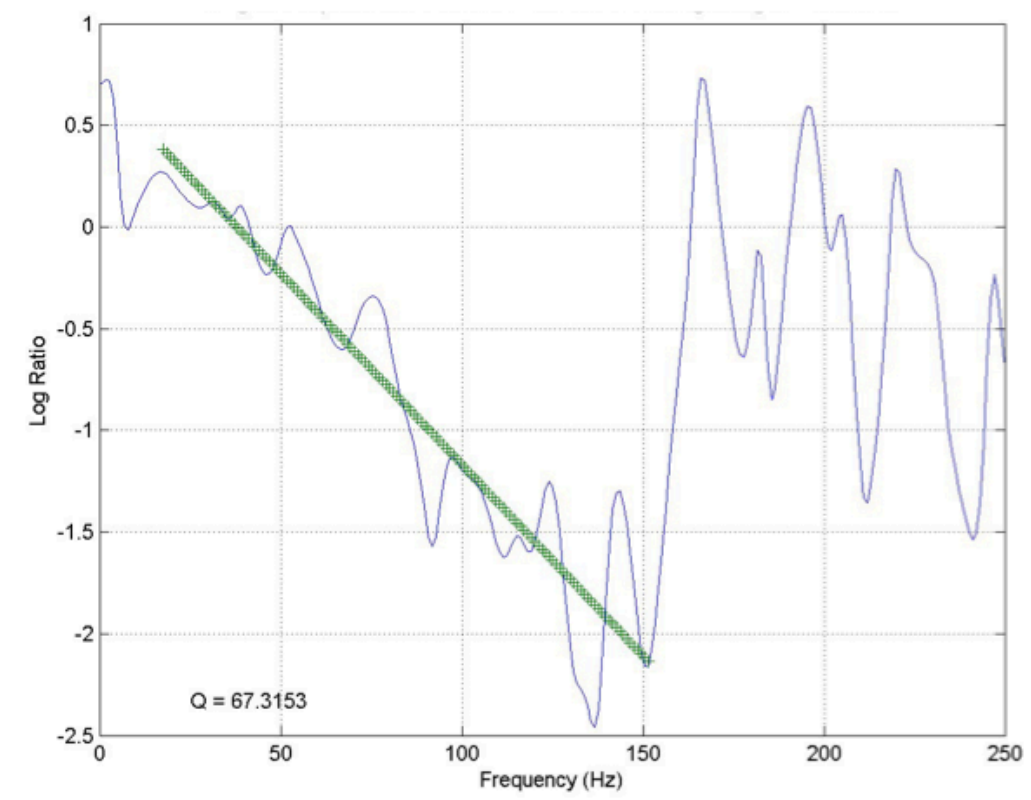

Figure 5.10: Spectral ratio plot of downgoing P-wave (Haase and Stewart, 2003). 


\section{Chapter 6}

\section{Conclusion}

The aim of this thesis has been to utilise the continuous wavelet transform in order to analyse and study the effects of absorption on a given pulse and subsequently establish an empirical relation between a functions regularity and the loss factor $Q$. An accurate estimation of the Lipschitz regularity of a seismic trace is regarded as a highly desirable goal, The continuous wavelet transform and the associated Lipschitz regularity provide a potentially efficient and powerful tool for analysing singularities in a signal. For a single event, a linear model enables us to estimate the Lipschitz exponent and characterise the singularity with relative ease.

For a single event, resembling a delta type function, one could use a linear model and subsequently estimate the associated Lipschitz estimate the Lipschitz exponent by finding the slope. Additionally, estimating the Lipschitz exponent associated with a Heaviside function and a ramp function confirms the theoretical properties of the Lipschitz regularity, such that the integration increased the Lipschitz value by 1 . Furthermore, it has been shown that the MHZ signal model can not provide an accurate Lipschitz estimation for two closely spaced events as the associated modulus maxima values merge, hence representing a single event rather than two distinct seismic events.

For practical applications and in order to accurately represent a seismic pulse that experiences attenuation, a signal would have to be modelled as a delta function smoothed by

a Gaussian (with variance $\sigma^{2}$ ), thus leading to a non-linear model. In order to estimate the Lipschitz exponent, one would have to impose some sort of thresholding technique, form the objective function and minimise using a relatively time consuming and computationally expensive method such as the steepest descent or re-arrange the MHZ model and minimise using the least squares method. 
For synthetic VSP modelled events, the steepest descent method fails to provide any information related to the smoothness parameter $\sigma$. A possible explanation could be related to the topography of the objective function, however without further investigation one can not state with certainty whether the topography limits the accuracy or efficiency of the method. Furthermore, as $Q$ decreases the steepest descent method provides estimates for the Lipschitz exponent $\alpha$ that seems suspicious.

By imposing some sort of thresholding, one could linearise the problem, eliminate two of the parameters ( $A$ and $\alpha$ ) and solely focus on estimating the Lipschitz exponent $\alpha$. However this method is prone to errors of visual bias. Re-arranging the non-linear MHZ signal model and minimising by using the least squares method, one obtains relatively accurate values for all three parameters (regularity, smoothness and amplitude) corresponding to respective VSP events.

An accurate estimation of signal regularity and smoothness for varying $Q$ values (corresponding to VSP model) provides an opportunity to mathematically map $Q$ to $\alpha$ and $\sigma$. Plotting the $\alpha$ against a range $Q$ values corresponding to a single receiver within the VSP Model, does not provide a trivial mathematical relation. Furthermore, the obtained mathematical function (mapping $Q$ and $\alpha$ ) is limited to a certain range of $Q$ values or absorption levels. However, plotting the smoothness parameter $\sigma$ against $Q$ does provide a relatively trivial, power law type relation between the two parameters. Additionally, inverting the obtained relation, does provide accurate $Q$ values in comparison to the original values.

Applying the obtained mathematical relation between $\sigma$ and $Q$ (from synthetic VSP modelling) to the Ross Lake field data provides mixed results. Compared to the spectral ratio method, the $Q$ values estimated from the obtained power law relation falls short. However, a positive and encouraging sign relates to the fact that the re-arranged non-linear MHZ model does capture the effects of absorption on a seismic signal. Hence, a theoretical and practical framework exists, such that additional research could potentially lead to a 
robust mathematical relation between smoothness and $Q$. 


\section{Bibliography}

Aki, R., and Richards, P., 2002, Quantitative Seismology: University Science Books, Sausalito California, 2 edn.

Aster, R. C., Borchers, B., and Clifford, T. H., 2005, Parameter Estimation and Inverse Problems: Elsevier Academic Press, 1 edn.

Balch, A. H., and Myung, L. W., 1984, Vertical Seismic Profiling: Techniques, Applications and Case Histories: International Human Resources Development Corporation.

Brigham, E. O., 1988, The Fast Fourier Transform and Its Applications: Prentice Hall, 1 edn.

Burden, R. L., and Douglas, F. J., 2005, Numerical Analysis: Thomson Books/Cole, 8 edn.

Christopher, J. E., 1974, The Upper Jurassic Vanguard and Lower Cretaceous Mannville Groups of Southwestern Saskatchewan, Tech. Rep. 151, Department of Mineral Resources: Saskatchewan Geological Survey, Sedimentary Geology Division.

Cohen, L., 1995, Time-Frequency Analysis: Prentice Hall.

Daubechies, I., 1992, Ten lectures on wavelets: Society for Industrial and Applied Mathematics.

Farge, M., 1992, Wavelet transform and their applications to turbulence: Annual Reviews Fluid Mechanics, 395-457.

Gao, R. E., and Yan, R., 2011, Wavelets: Theory and Applications for Manufacturing: Springer.

Haase, A. B., and Stewart, R. R., 2003, Q-factor estimation from borehole seismic data: Ross Lake, Saskatchewan, Crewes research report, University of Calgary. 
Haase, A. B., and Stewart, R. R., 2004, Attenuation Estimates from VSP and Log Data: Society of Exploration Geophysicists.

Hardage, B. A., 1983, Vertical Seismic Profiling Part A: Principles, vol. 14A: Geophysical Press Limited.

Hermann, F. J., 1997, A scaling medium representation, a discussion on well-logs, fractals and waves: Ph.D. thesis, Technische Universiteit Delft.

Hinds, R. C., Anderson, N. L., and Kuzmiski, R. D., 1996, VSP Interpretive Processing: Theory and Practice: Society of Exploration Geophysicists.

Hogan, J. A., and Lakey, J. D., 2003, Time-Frequency and Time-Scale Methods: Adaptive Decompositions, Uncertainty Principle and Sampling: Birkhauser.

Hong, J., Kim, Y., and Lee, Y. W., 2002, Damage detection using the Lipschitz exponent estimated by the wavelet transform: applications to vibration model of a beam: International Journal of Solids and Structures, 39.

Innanen, K. A., 2003, Local signal regularity and Lipschitz exponents as a means to estimate Q: Seismic Exploration, , No. 12, 53-74.

Kaiser, G. A., 1994, Friendly Guide to Wavelets: Birkhauser.

Kennett, P., Ireson, R. L., and Conn, P. J., 1980, Vertical seismic profiles: Their applications in exploration geophysics: Geophysical Prospecting, 28, 676-699.

Kjartansson, E., 1979, Attenuation of seismic waves in rocks and applications in energy exploration: Ph.D. thesis, Stanford University.

Lines, L., Vasheghani, F., and Treitel, S., 2008, Reflections on Q.

Mallat, S., 2009, a Wavelet tour of signal processing, The Sparse Way: Elsevier, 3 edn. 
Mallat, S., and Hwang, L., 1992, Singularity detection and processing with wavelets: IEEE Transactions on Information Theory, 38, No. 2.

Mallat, S., and Zhong, S., 1992, Characterisation of signals from multiscale edges: IEEE Transactions on Pattern Analysis and Machine Intelligence, 14, No. 7.

Meza, J. C., 2010, Steepest Descent: Wiley Interdisciplinary Reviews: Computational Statistics, 2, 719-722.

Mittet, R., Sollie, R., and Hokstad, K., 1995, Prestack depth migration with compensation for absorption and dispersion: Geophysics, 60, No. 5 .

Nicholson, K. W., 1990, Linear Algebra With Applications: Pearson Publishing Company, 3 edn.

Okamura, S., 2011, The Short Time Fourier Transform and Local Signals: Ph.D. thesis, Carnengie Mellon University.

Qian, S., 2002, Introduction to Time-Frequency and Wavelet Transforms: Prentice Hall.

Quan, Y., and Harris, J. M., 1997, Seismic attenuation tomography using the frequency shift method: Geophysics, 62, No. 3.

Sergio, L. M. F., and Ulrych, T., 1988, Application of singular value decompostion to vertical sesmic profiling: Geophysics, 53, No. 6, 778.

Sheng, Y., 2000, The Transforms and Applications Handbook, Chapter 10: CRC Press, 2 edn.

Simman, M., 2007, Advances in geophysical data processing: Vertical seismic profiles: Geophysics Journal of the Royal Astronomical Society, 86, No. 1, 216-217.

Strick, E., 1970, A predicted pedestal effect for pulse propagation in constant Q solids: Geophysics, , No. 35, 387-403. 
Tonn, R., 1991, The Determination of the Seismic Quality Factor Q From VSP Data: A Comparison of Different Computational Methods: Geophysical Prospecting, , No. 39, 127.

Wang, X., 2008, Method of Stepest Descent and its Applications, Department of Engineering, University of Tennessee, Knoxville, TN 37996.

Zhang, C., 2008, Seismic absorption estimation and compensation: Ph.D. thesis, University of British Columbia.

Zhang, Z., 2010, Assessing attenuation, fractures, and anisotropy using logs, vertical seismic profile, and three-component seismic data: heavy oilfield and potash mining examples: Ph.D. thesis, University of Calgary.

Zhang, Z., and Stewart, R. R., 2006, VSP processing and analysis at the Ross Lake heavy oilfield, Saskatchewan, Crewes research report, Univerity of Calgary. 